\title{
In Situ Ligand Formations in the Synthetic Processes from Mononuclear Dy (III) Compounds to Binuclear Dy (III) Compounds: Synthesis, Structure, Magnetic Behavior and Theoretical Analysis
}

Sheng Zhang, ${ }^{* a}$ Jiamin Tang, ${ }^{a}$ Jin Zhang, ${ }^{a}$ Fang $\mathrm{Xu},{ }^{\text {a }}$ Sanping Chen, ${ }^{* b}$ Dengwei $\mathrm{Hu},{ }^{\mathrm{a}}$ Bing Yin, ${ }^{* b}$ Jiangwei Zhang*c

\section{AUTHOR ADDRESS}

a. Faculty of Chemistry and Chemical Engineering, Engineering Research Center of Advanced Ferroelectric Functional Materials, Key Laboratory of Phytochemistry of Shaanxi Province, Baoji University of Arts and Sciences, 1 Hi-Tech Avenue, Baoji, Shaanxi, 721013, China

${ }^{b}$.Key Laboratory of Synthetic and Natural Functional Molecule Chemistry of Ministry of Education, College of Chemistry and Materials Science, Northwest University, Xi'an, Shaanxi 710069, China

c.Dalian National Laboratory for Clean Energy \& State Key Laboratory of Catalysis, Dalian Institute of Chemical Physics, Chinese Academy of Sciences (CAS) Dalian 11 6023, P.R.China

\section{Corresponding Authors}

*E-mail: zhangsheng19890501@163.com

*E-mail: sanpingchen@126.com

*E-mail: rayinyin@nwu.edu.cn

*E-mail: jwzhang@dicp.ac.cn 


\section{Materials and instruction}

The FT-IR spectra were recorded in the range of $400-4000 \mathrm{~cm}^{-1}$ using $\mathrm{KBr}$ pellets on an EQUINOX55 FT/IR spectrophotometer. Elemental analysis $(\mathrm{C}, \mathrm{H}, \mathrm{N})$ was implemented on a Perkin-Elmer $2400 \mathrm{CHN}$ elemental analyzer. The phase purity of the bulk or polycrystalline samples were confirmed by powder X-ray diffraction (PXRD) measurements executed on a Rigaku RU200 diffractometer at $60 \mathrm{kV}, 300$ $\mathrm{mA}$, and $\mathrm{Cu} \mathrm{K} \alpha$ radiation $(\lambda=1.5406 \AA)$, with a scan speed of $5^{\circ} \mathrm{min}^{-1}$ and a step size of $0.02^{\circ}$ in $2 \theta$. All NMR spectra were obtained at ambient temperature using Bruker-AVANCE III-400MHz spectrometer. ${ }^{1} \mathrm{H}$ NMR and ${ }^{13} \mathrm{C}$ NMR spectra were recorded using $\mathrm{CDCl}_{3}$ as solvent. High resolution mass spectrometry (HRMS) data were obtained on a Micro TOF-Q (hybrid quadrupolar/time II -of -flight) API US system by electrospray ionization (ESI) in the positive ion mode using a Bruker instrument. The thermal behaviours of compounds 1-4 were investigated by thermogravimetric analyzer (TG, TG209F3 Tarsus) at a heating rate of $10{ }^{\circ} \mathrm{C} / \mathrm{min}$ in the $\mathrm{N}_{2}$. Magnetic measurements were performed in the temperature range 2.0-300 K with an applied field of 1000 Oe, using a Quantum Design MPMS-XL-7 SQUID magnetometer on polycrystalline samples. The diamagnetic corrections for the compounds were estimated using Pascal's constants. Alternating current (ac) susceptibility experiments were performed using an oscillating ac field of 2.0 Oe at ac frequencies ranging from 1 to $1000 \mathrm{~Hz}$. The magnetization was measured in the field range $0-7 \mathrm{~T}$.

\section{X-ray single-crystal diffraction analysis}

The single crystal X-ray experiment was performed on a Agilent Xcalibur Eos Gemini diffractometer equipped with a graphite-monochromatized $\mathrm{Cu} \mathrm{K} \alpha$ radiation $(\lambda$ $=1.5418 \AA$ ). The data integration and reduction were processed with CrysAlisPro software. Absorption correction based on multiscan was performed using the SADABS program. The structures were solved by the direct method and refined by means of full-matrix least-squares procedures on $F^{2}$ with SHELXL-2018 program. All nonhydrogen atoms were refined anisotropically. In $\mathbf{2}$, due to the fact that the guest 
molecules in the crystal are highly disordered and impossible to refine using conventional discrete-atom models, the SQUEEZE subroutine of the PLATON software suite was applied to remove the scattering from the highly disordered solvent molecules, and the sets of solvent-free diffraction intensities were produced. The PLATON squeeze process details were also provided in ESI. Note that in compound 2 structure with heavy-atom of Dy as it was not possible to see clear electron-density peaks in difference maps which would correspond with acceptable locations for the hydroxy $\mathrm{H}$ atoms bonded to $\mathrm{O}_{3}$ and $\mathrm{O}_{10}$. The hydroxy $\mathrm{H}$ atoms in compound 2 were added and refined geometrically. Other details of crystal data, data collection parameters and refinement statistics are given in Table 1. The selected bond lengths and angles are listed in Table S1.

\section{The synthesis ligand $\mathrm{H}_{2} \mathrm{~L}$}

\subsection{2-Hydroxy-3-(chloromethyl)-5-methyl-benzaldehyde (HCMB)}

$\mathrm{HCMB}$ was prepared by a direct chloromethylation of $\mathrm{HMB}$. In a $250 \mathrm{~mL}$ round bottom flask, a mixture of $6.4 \mathrm{~g}(4.7 \mathrm{mmol})$ of $\mathrm{HMB}, 7.5 \mathrm{~mL}$ of formaldehyde and 25 $\mathrm{mL}$ of $\mathrm{HCl}(12 \mathrm{~mol} / \mathrm{L})$ was heated under magnetic stirring to reflux for $15 \mathrm{~min}$. After cool down the reaction to $0{ }^{\circ} \mathrm{C}$, a solid mass was formed in the bottom. The solid was separated from the reaction solution and recrystallized in hot ethanol, yielding $5 \mathrm{~g}$ of HCMB (70\% yield). The residual solutions were basified with $\mathrm{NaOH}(\mathrm{pH}>9)$ prior to discard them. ${ }^{1} \mathrm{H}$ NMR $\left(400 \mathrm{MHz}, \mathrm{CDCl}_{3}\right) \delta 11.24(\mathrm{~s}, 1 \mathrm{H}), 9.80(\mathrm{~s}, 1 \mathrm{H}), 7.42$ (d, $J$ $=2.1 \mathrm{~Hz}, 1 \mathrm{H}), 7.30(\mathrm{~d}, J=1.4 \mathrm{~Hz}, 1 \mathrm{H}), 4.64(\mathrm{~s}, 2 \mathrm{H}), 2.31(\mathrm{~d}, J=4.4 \mathrm{~Hz}, 3 \mathrm{H}) .{ }^{1} \mathrm{H}$ NMR $\left(100 \mathrm{MHz}, \mathrm{CDCl}_{3}\right) \delta 196.7,196.5,159.5,157.3,138.6,138.1,134.1,133.5$, $129.3,129.2,125.7,120.4,120.4,117.4,39.9,20.3,20.2$. 


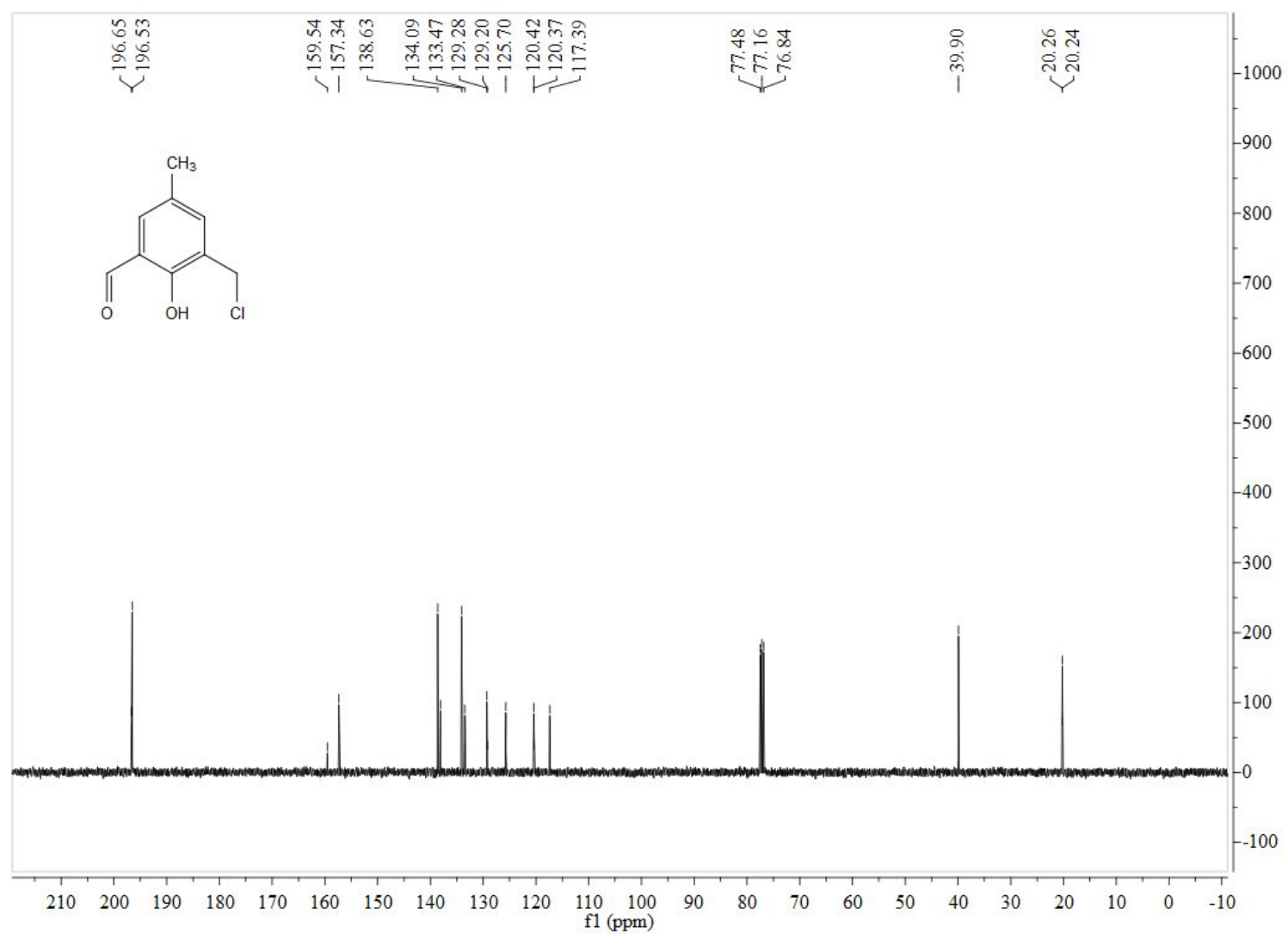

Figure S1. ${ }^{13} \mathrm{C}$ NMR spectra of HCMB.

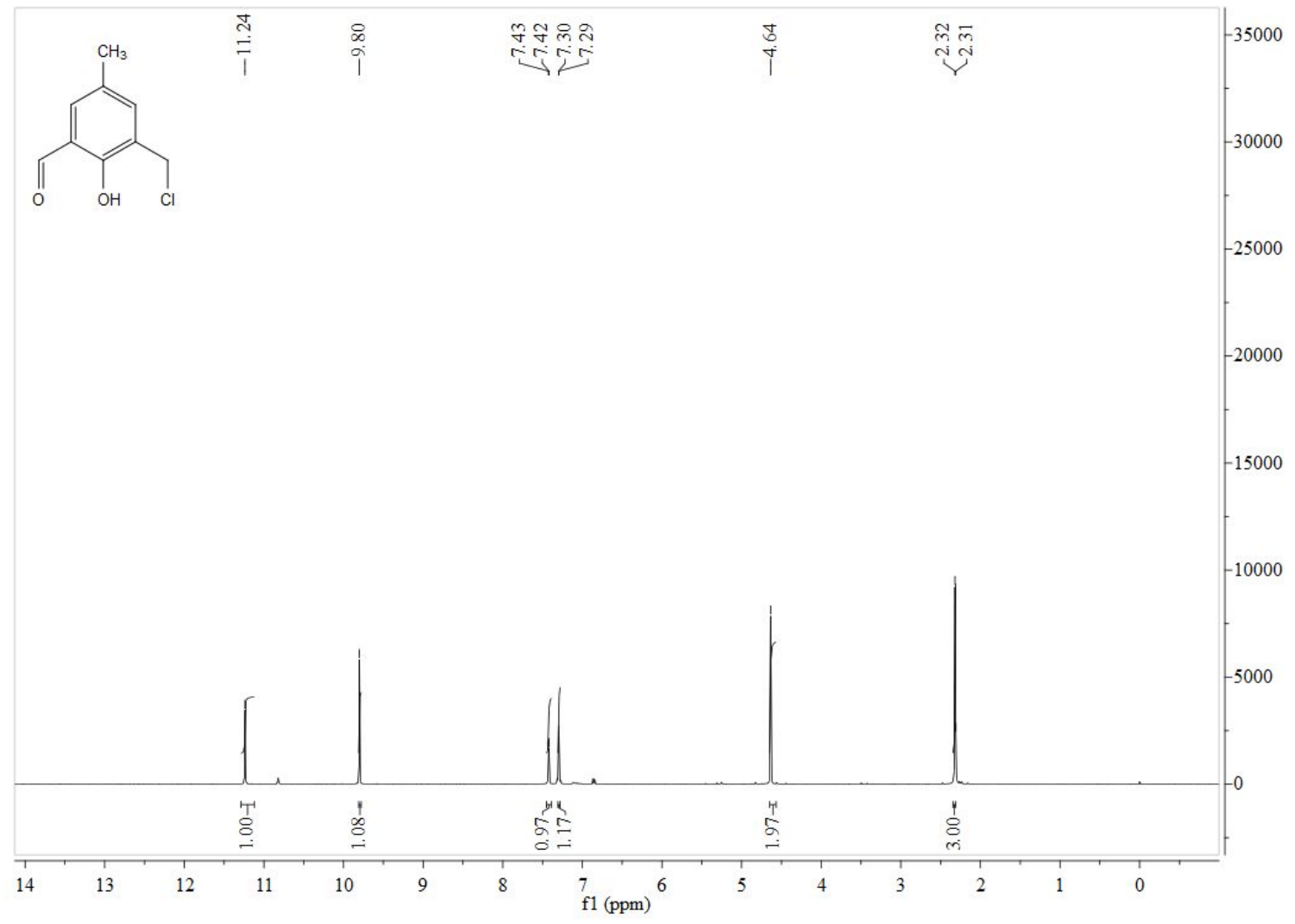

Figure S2. ${ }^{1} \mathrm{H}$ NMR spectra of HCMB. 
1.2 N,N'-bis(pyridin-2-ylmethyl)ethylenediamine ( $\left.\mathrm{py}_{2} \mathrm{en}\right)$

$\mathrm{Py}_{2}$ en was prepared by a condensation reaction between 2-pyridinecarboxaldehyde and ethylenediamine, followed by reduction with $\mathrm{NaBH}_{4}$. In a $100 \mathrm{~mL}$ round bottom flask, $3.0 \mathrm{~mL}$ of pyridine-2-carboxaldehyde $(31 \mathrm{mmol})$ were dissolved in $30 \mathrm{~mL}$ of methanol at $0{ }^{\circ} \mathrm{C}$, followed by the addition of $1.0 \mathrm{~mL}$ of ethylenediamine $(15.5$ mmol), under magnetic stirring. After $1 \mathrm{~h}, 1.2 \mathrm{~g}$ of sodium borohydride (31 mmol) was added in small portions and left to react for another $1 \mathrm{~h}$. Then, $\mathrm{HCl} 6 \mathrm{~mol} / \mathrm{L}$ was added dropwise up to $\mathrm{pH}<2$. After removing the solvent, $30 \mathrm{~mL}$ of distilled water was added and the $\mathrm{pH}$ adjusted to $9-10$ with $\mathrm{NaOH}$. The product was extracted with dichloromethane $(6 \times 50 \mathrm{~mL})$; the organic layers were dried under MgSO4 and the solvent was removed under vacuum, yielding $2.8 \mathrm{~g}$ of a yellow oil of py $\mathrm{z}_{2}$ en $(85 \%$ yield). ${ }^{1} \mathrm{H}$ NMR (400 MHz, $\left.\mathrm{CDCl}_{3}\right) \delta 8.57-8.51(\mathrm{~m}, 2 \mathrm{H}), 7.62(\mathrm{td}, J=7.7,1.8 \mathrm{~Hz}$, 2H), 7.32 (d, $J=7.8 \mathrm{~Hz}, 2 \mathrm{H}), 7.14$ (ddd, $J=7.4,4.9,0.9 \mathrm{~Hz}, 2 \mathrm{H}), 3.92$ (s, 4H), 2.82 (s, 4H). ${ }^{13} \mathrm{C} \mathrm{NMR}\left(100 \mathrm{MHz}, \mathrm{CDCl}_{3}\right) \delta 159.8,149.1,136.3,122.1,121.7,55.1,49.0$.

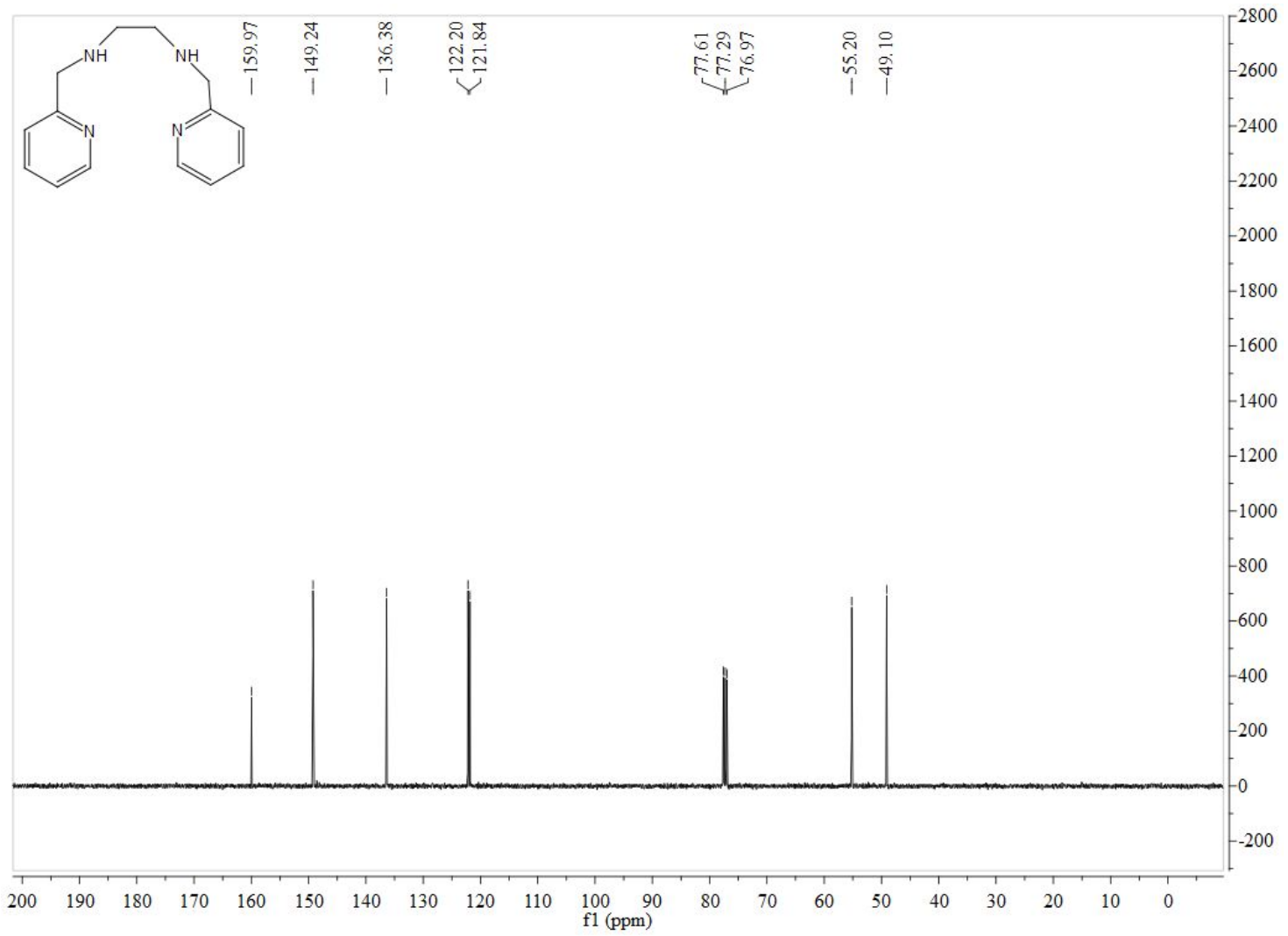

Figure S3. ${ }^{13} \mathrm{C}$ NMR spectra of $\mathrm{py}_{2} \mathrm{en}$. 


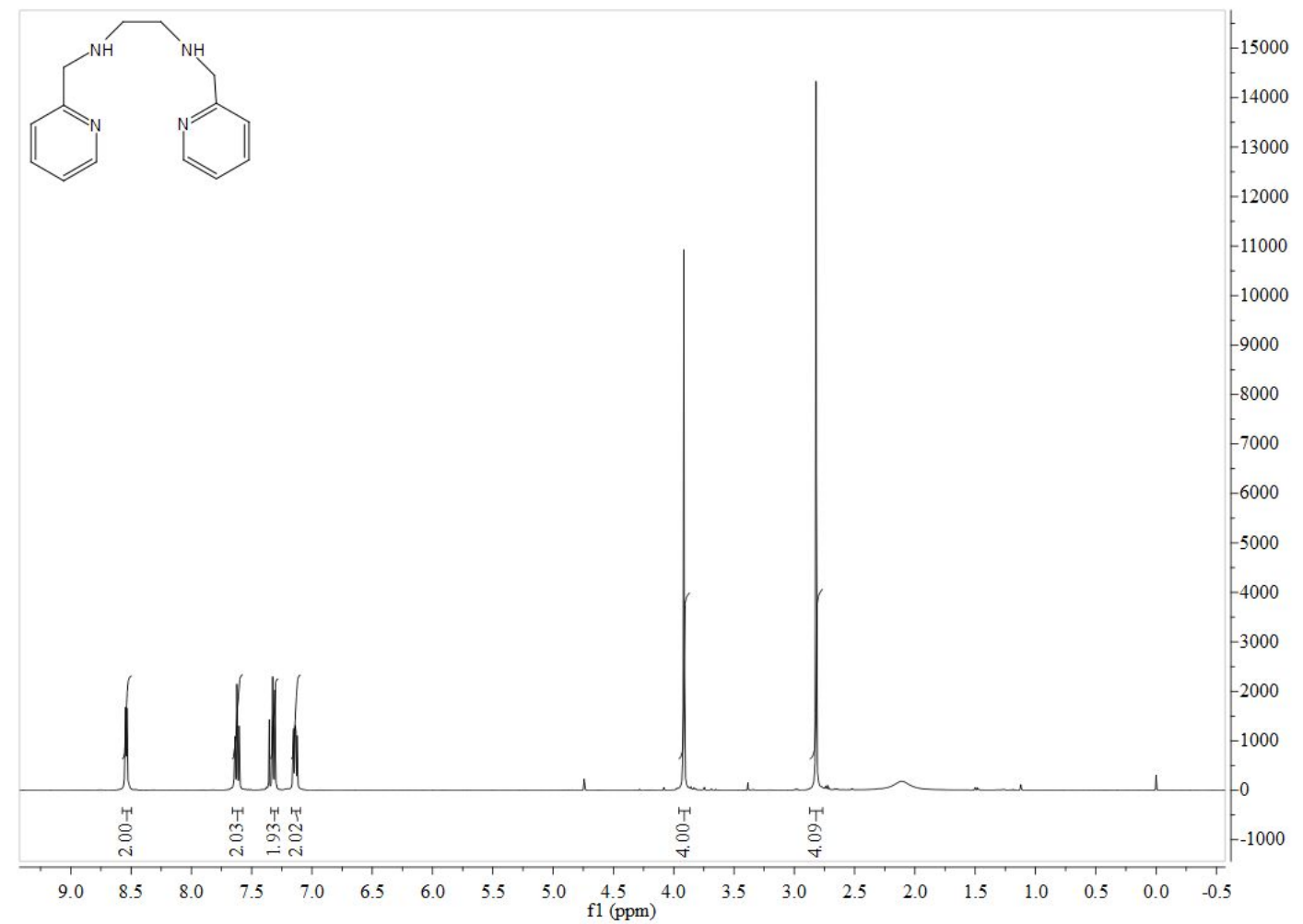

Figure S4. ${ }^{1} \mathrm{H}$ NMR spectra of HCMB.

\section{3}

$\mathrm{N}, \mathrm{N}^{\prime}$-bis(2-hydroxy-5-methyl-3-formylbenzyl)-N,N'-bis-(pyridin-2-ylmethyl)ethylene diamine, $\mathrm{H}_{2} \mathrm{~L}$

In a $100 \mathrm{~mL}$ round bottom flask, $4.6 \mathrm{~g}$ of $\mathrm{HCMB}(25 \mathrm{mmol})$ and $3.0 \mathrm{~g}$ of $\mathrm{py}_{2} \mathrm{en}(12$ mmol) were dissolved in $40 \mathrm{~mL}$ of dichloromethane. Then, $3.5 \mathrm{~mL}$ of triethylamine ( $25 \mathrm{mmol}$ ) was added dropwise. After $24 \mathrm{~h}$ at room temperature, the reaction solution was washed with a saturated solution of $\mathrm{NaHCO}_{3}$ and the organic layers dried with $\mathrm{MgSO}_{4}$. After removing the solvent under vacuum, $6.4 \mathrm{~g}$ of $\mathrm{H}_{2} \mathrm{~L}$ were obtained, as viscous yellow oil (96\% yield). ${ }^{1} \mathrm{H}$ NMR $\left(400 \mathrm{MHz}, \mathrm{CDCl}_{3}\right) \delta 10.19(\mathrm{~d}, J=2.2 \mathrm{~Hz}$, 2H), $8.53(\mathrm{dd}, J=2.8,2.0 \mathrm{~Hz}, 2 \mathrm{H}), 7.59$ (dd, $J=10.6,4.7 \mathrm{~Hz}, 2 \mathrm{H}), 7.37$ (s, 2H), 7.25 (d, $J=7.7 \mathrm{~Hz}, 2 \mathrm{H}), 7.16(\mathrm{dd}, J=12.7,7.6 \mathrm{~Hz}, 4 \mathrm{H}), 3.74(\mathrm{~s}, 4 \mathrm{H}), 3.66$ (s, 4H), 2.75 (s, 4H), $2.23(\mathrm{~s}, 6 \mathrm{H}) .{ }^{13} \mathrm{C} \mathrm{NMR}\left(100 \mathrm{MHz}, \mathrm{CDCl}_{3}\right) \delta 192.6,159.0,157.9,148.9,137.5$, 136.7, 128.8, 128.1, 125.0, 123.2, 122.3, 122.2, 59.4, 54.9, 50.5, 20.3. HRMS (ESI) for $\mathrm{C}_{32} \mathrm{H}_{35} \mathrm{~N}_{4} \mathrm{O}_{4}\left[\mathrm{M}+\mathrm{H}^{+}\right]$: Calcd: 539.2209; Found: 539.2202. IR (KBr): 2958, 2844, $1590,1471,1240,1083,989,871$. 




Figure S5. ${ }^{13} \mathrm{C}$ NMR spectra of $\mathrm{H}_{2} \mathrm{~L}$.

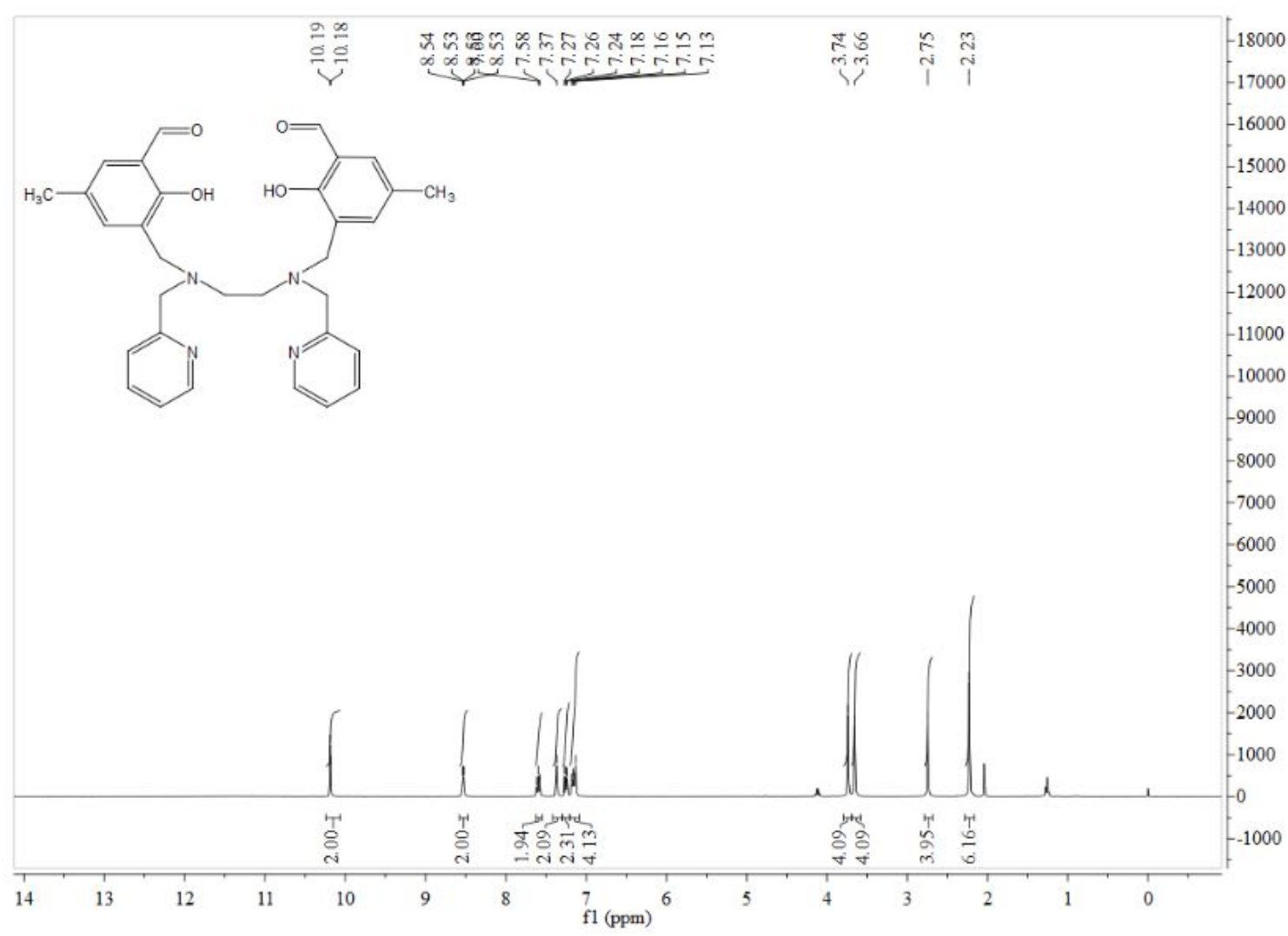

Figure S6. ${ }^{1} \mathrm{H}$ NMR spectra of $\mathrm{H}_{2} \mathrm{~L}$. 


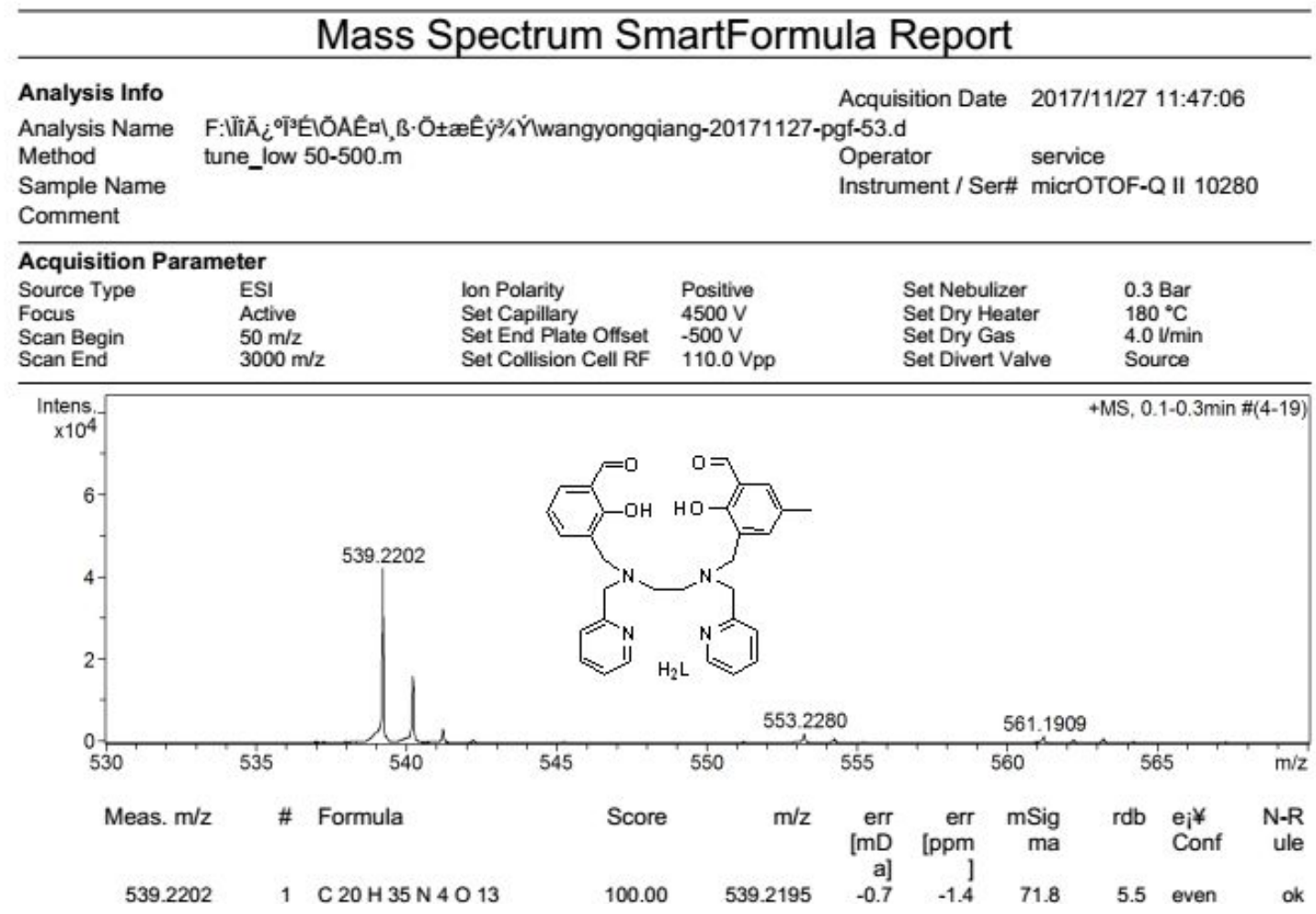

Figure S7. HRMS spectra of $\mathrm{H}_{2} \mathrm{~L}$.

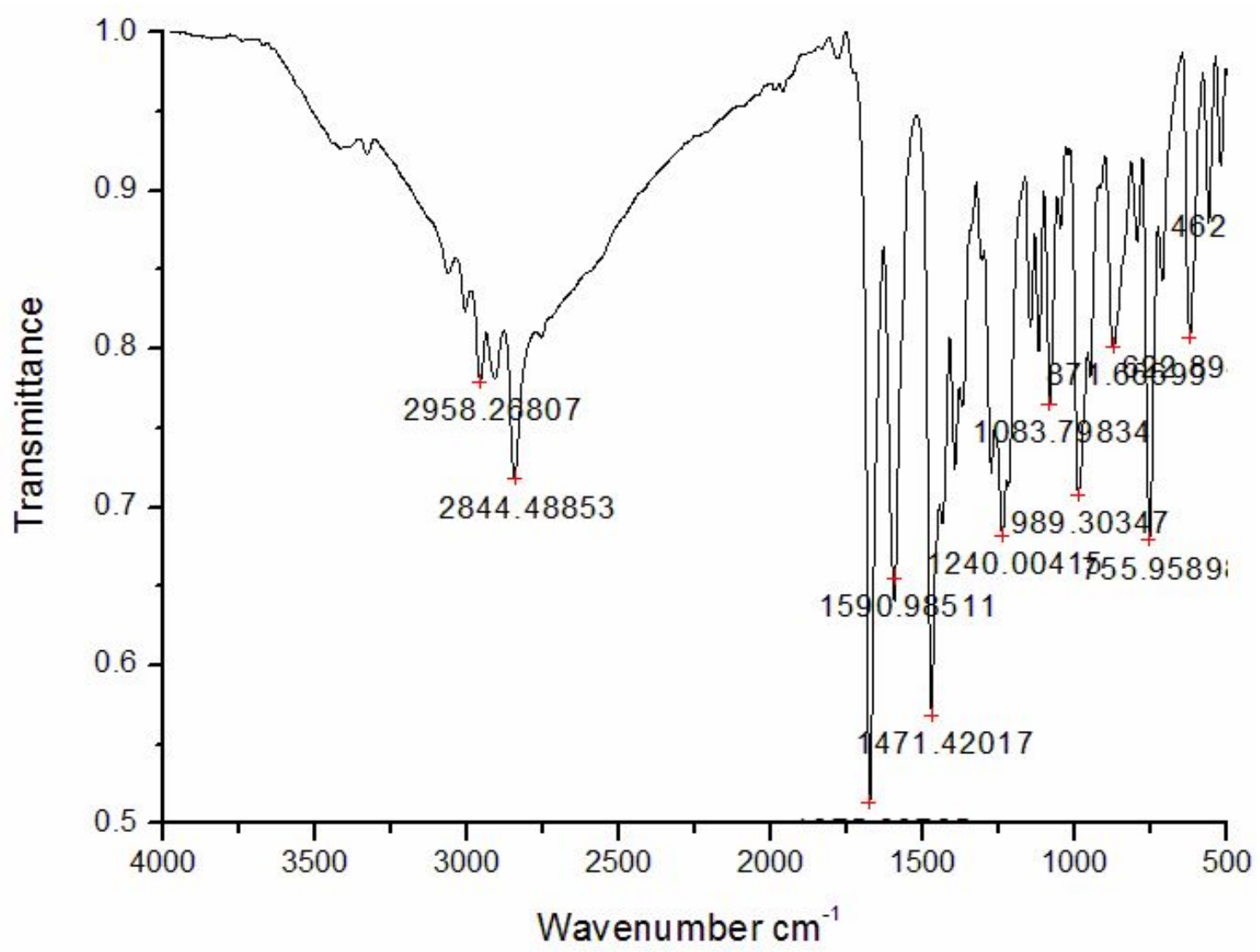

Figure S8. FTIR spectra of $\mathrm{H}_{2} \mathrm{~L}$.

\section{The structure information of 1-4}


Table S1. Selected bond lengths $(\AA)$ and angles $\left(^{\circ}\right)$ for 1-4.

\begin{tabular}{|c|c|c|c|}
\hline \multicolumn{4}{|l|}{ Compound $\mathbf{1}$} \\
\hline $\mathrm{Dy}(1)-\mathrm{O}(4)$ & $2.322(3)$ & $\mathrm{O}(2)-\mathrm{Dy}(1)-\mathrm{N}(5)$ & $76.96(11)$ \\
\hline $\mathrm{Dy}(1)-\mathrm{O}(2)$ & $2.229(3)$ & $\mathrm{O}(5)-\mathrm{Dy}(1)-\mathrm{O}(4)$ & $84.69(11)$ \\
\hline $\mathrm{Dy}(1)-\mathrm{O}(5)$ & $2.267(3)$ & $\mathrm{O}(5)-\mathrm{Dy}(1)-\mathrm{O}(3)$ & $92.03(12)$ \\
\hline $\mathrm{Dy}(1)-\mathrm{O}(3)$ & $2.321(3)$ & $\mathrm{O}(5)-\mathrm{Dy}(1)-\mathrm{N}(4)$ & $74.45(10)$ \\
\hline Dy(1)-N(4) & $2.628(3)$ & $\mathrm{O}(5)-\mathrm{Dy}(1)-\mathrm{N}(3)$ & $133.24(10)$ \\
\hline Dy(1)-N(3) & $2.616(3)$ & $\mathrm{O}(5)-\mathrm{Dy}(1)-\mathrm{N}(6)$ & $75.29(11)$ \\
\hline $\operatorname{Dy}(1)-N(6)$ & $2.590(4)$ & $\mathrm{O}(5)-\mathrm{Dy}(1)-\mathrm{N}(5)$ & $92.17(11)$ \\
\hline $\operatorname{Dy}(1)-N(5)$ & $2.621(3)$ & $\mathrm{O}(3)-\mathrm{Dy}(1)-\mathrm{O}(4)$ & $71.31(10)$ \\
\hline $\mathrm{O}(4)-\mathrm{Dy}(1)-\mathrm{N}(4)$ & $78.25(10)$ & $\mathrm{O}(3)-\mathrm{Dy}(1)-\mathrm{N}(4)$ & $147.73(11)$ \\
\hline $\mathrm{O}(4)-\mathrm{Dy}(1)-\mathrm{N}(3)$ & $69.72(11)$ & $\mathrm{O}(3)-\mathrm{Dy}(1)-\mathrm{N}(3)$ & $114.18(12)$ \\
\hline $\mathrm{O}(4)-\mathrm{Dy}(1)-\mathrm{N}(6)$ & $141.90(11)$ & $\mathrm{O}(3)-\mathrm{Dy}(1)-\mathrm{N}(6)$ & $77.28(11)$ \\
\hline $\mathrm{O}(4)-\mathrm{Dy}(1)-\mathrm{N}(5)$ & $148.27(10)$ & $\mathrm{O}(3)-\mathrm{Dy}(1)-\mathrm{N}(5)$ & $140.42(11)$ \\
\hline $\mathrm{O}(2)-\mathrm{Dy}(1)-\mathrm{O}(4)$ & $118.38(11)$ & $\mathrm{N}(3)-\mathrm{Dy}(1)-\mathrm{N}(4)$ & $62.68(10)$ \\
\hline $\mathrm{O}(2)-\mathrm{Dy}(1)-\mathrm{O}(5)$ & $151.17(10)$ & $\mathrm{N}(3)-\mathrm{Dy}(1)-\mathrm{N}(5)$ & $90.53(11)$ \\
\hline $\mathrm{O}(2)-\mathrm{Dy}(1)-\mathrm{O}(3)$ & $80.65(12)$ & $\mathrm{N}(6)-\mathrm{Dy}(1)-\mathrm{N}(4)$ & $124.94(11)$ \\
\hline $\mathrm{O}(2)-\mathrm{Dy}(1)-\mathrm{N}(4)$ & $124.51(11)$ & $\mathrm{N}(6)-\mathrm{Dy}(1)-\mathrm{N}(3)$ & $145.40(11)$ \\
\hline $\mathrm{O}(2)-\mathrm{Dy}(1)-\mathrm{N}(3)$ & $74.24(11)$ & $\mathrm{N}(6)-\mathrm{Dy}(1)-\mathrm{N}(5)$ & $65.88(11)$ \\
\hline $\mathrm{O}(2)-\mathrm{Dy}(1)-\mathrm{N}(6)$ & $75.89(11)$ & $\mathrm{N}(5)-\mathrm{Dy}(1)-\mathrm{N}(4)$ & $70.53(10)$ \\
\hline \multicolumn{4}{|l|}{ Compound $\mathbf{2}$} \\
\hline $\mathrm{Dy}(1)-\mathrm{O}(7)$ & $2.237(10)$ & $\mathrm{O}(1)-\mathrm{Dy}(1)-\mathrm{N}(1)$ & $75.1(4)$ \\
\hline $\mathrm{Dy}(1)-\mathrm{O}(4)$ & $2.268(10)$ & $\mathrm{O}(8)-\mathrm{Dy}(1)-\mathrm{N}(1)$ & $141.6(4)$ \\
\hline $\mathrm{Dy}(1)-\mathrm{O}(2)$ & $2.288(9)$ & $\mathrm{O}(9)-\mathrm{Dy}(1)-\mathrm{N}(1)$ & $104.9(4)$ \\
\hline $\mathrm{Dy}(1)-\mathrm{O}(1)$ & $2.299(10)$ & $\mathrm{N}(6)-\mathrm{Dy}(1)-\mathrm{N}(1)$ & $77.3(4)$ \\
\hline $\mathrm{Dy}(1)-\mathrm{O}(8)$ & $2.329(10)$ & $\mathrm{O}(7)-\mathrm{Dy}(1)-\mathrm{Dy}(2)$ & $116.0(2)$ \\
\hline $\mathrm{Dy}(1)-\mathrm{O}(9)$ & $2.380(9)$ & $\mathrm{O}(4)-\mathrm{Dy}(1)-\mathrm{Dy}(2)$ & $32.4(2)$ \\
\hline $\operatorname{Dy}(1)-N(6)$ & $2.552(13)$ & $\mathrm{O}(2)-\mathrm{Dy}(1)-\mathrm{Dy}(2)$ & $112.4(2)$ \\
\hline Dy $(1)-\mathrm{N}(1)$ & $2.601(13)$ & $\mathrm{O}(1)-\mathrm{Dy}(1)-\mathrm{Dy}(2)$ & $153.8(3)$ \\
\hline $\operatorname{Dy}(1)-\operatorname{Dy}(2)$ & $3.8843(14)$ & $\mathrm{O}(8)-\mathrm{Dy}(1)-\mathrm{Dy}(2)$ & $87.4(2)$ \\
\hline $\mathrm{Dy}(2)-\mathrm{O}(6)$ & $2.298(10)$ & $\mathrm{O}(9)-\mathrm{Dy}(1)-\mathrm{Dy}(2)$ & $32.3(2)$ \\
\hline $\mathrm{Dy}(2)-\mathrm{O}(9)$ & $2.263(10)$ & $\mathrm{N}(6)-\mathrm{Dy}(1)-\mathrm{Dy}(2)$ & $85.3(3)$ \\
\hline $\mathrm{Dy}(2)-\mathrm{O}(5)$ & $2.282(10)$ & $\mathrm{N}(1)-\mathrm{Dy}(1)-\mathrm{Dy}(2)$ & $81.6(3)$ \\
\hline $\mathrm{Dy}(2)-\mathrm{O}(4)$ & $2.315(9)$ & $\mathrm{O}(6)-\mathrm{Dy}(2)-\mathrm{O}(9)$ & $79.9(3)$ \\
\hline $\operatorname{Dy}(2)-N(5)$ & $2.531(12)$ & $\mathrm{O}(6)-\mathrm{Dy}(2)-\mathrm{O}(5)$ & $72.5(4)$ \\
\hline $\operatorname{Dy}(2)-N(3)$ & $2.553(14)$ & $\mathrm{O}(9)-\mathrm{Dy}(2)-\mathrm{O}(5)$ & $97.2(4)$ \\
\hline $\mathrm{Dy}(2)-\mathrm{N}(4)$ & $2.576(9)$ & $\mathrm{O}(6)-\mathrm{Dy}(2)-\mathrm{O}(4)$ & $134.2(3)$ \\
\hline $\operatorname{Dy}(2)-N(2)$ & $2.598(13)$ & $\mathrm{O}(9)-\mathrm{Dy}(2)-\mathrm{O}(4)$ & $65.1(3)$ \\
\hline $\mathrm{O}(7)-\mathrm{Dy}(1)-\mathrm{O}(4)$ & $142.8(3)$ & $\mathrm{O}(5)-\mathrm{Dy}(2)-\mathrm{O}(4)$ & $83.3(3)$ \\
\hline $\mathrm{O}(7)-\mathrm{Dy}(1)-\mathrm{O}(2)$ & $118.4(4)$ & $\mathrm{O}(6)-\mathrm{Dy}(2)-\mathrm{N}(5)$ & $80.5(4)$ \\
\hline $\mathrm{O}(4)-\mathrm{Dy}(1)-\mathrm{O}(2)$ & $80.5(3)$ & $\mathrm{O}(9)-\mathrm{Dy}(2)-\mathrm{N}(5)$ & $78.1(4)$ \\
\hline $\mathrm{O}(7)-\mathrm{Dy}(1)-\mathrm{O}(1)$ & $78.6(4)$ & $\mathrm{O}(5)-\mathrm{Dy}(2)-\mathrm{N}(5)$ & $153.0(4)$ \\
\hline
\end{tabular}




\begin{tabular}{|c|c|c|c|}
\hline $\mathrm{O}(4)-\mathrm{Dy}(1)-\mathrm{O}(1)$ & $138.6(3)$ & $\mathrm{O}(4)-\mathrm{Dy}(2)-\mathrm{N}(5)$ & $117.3(4)$ \\
\hline $\mathrm{O}(2)-\mathrm{Dy}(1)-\mathrm{O}(1)$ & $74.1(3)$ & $\mathrm{O}(6)-\mathrm{Dy}(2)-\mathrm{N}(3)$ & $121.9(4)$ \\
\hline $\mathrm{O}(7)-\mathrm{Dy}(1)-\mathrm{O}(8)$ & $73.1(4)$ & $\mathrm{O}(9)-\mathrm{Dy}(2)-\mathrm{N}(3)$ & $153.1(3)$ \\
\hline $\mathrm{O}(4)-\mathrm{Dy}(1)-\mathrm{O}(8)$ & $83.7(3)$ & $\mathrm{O}(5)-\mathrm{Dy}(2)-\mathrm{N}(3)$ & $77.1(4)$ \\
\hline $\mathrm{O}(2)-\mathrm{Dy}(1)-\mathrm{O}(8)$ & $73.5(3)$ & $\mathrm{O}(4)-\mathrm{Dy}(2)-\mathrm{N}(3)$ & $88.1(4)$ \\
\hline $\mathrm{O}(1)-\mathrm{Dy}(1)-\mathrm{O}(8)$ & $118.4(3)$ & $\mathrm{N}(5)-\mathrm{Dy}(2)-\mathrm{N}(3)$ & $118.4(4)$ \\
\hline $\mathrm{O}(7)-\mathrm{Dy}(1)-\mathrm{O}(9)$ & $84.3(3)$ & $\mathrm{O}(6)-\mathrm{Dy}(2)-\mathrm{N}(4)$ & $68.0(4)$ \\
\hline $\mathrm{O}(4)-\mathrm{Dy}(1)-\mathrm{O}(9)$ & $63.9(3)$ & $\mathrm{O}(9)-\mathrm{Dy}(2)-\mathrm{N}(4)$ & $133.6(4)$ \\
\hline $\mathrm{O}(2)-\mathrm{Dy}(1)-\mathrm{O}(9)$ & $139.2(3)$ & $\mathrm{O}(5)-\mathrm{Dy}(2)-\mathrm{N}(4)$ & $103.7(4)$ \\
\hline $\mathrm{O}(1)-\mathrm{Dy}(1)-\mathrm{O}(9)$ & $146.5(3)$ & $\mathrm{O}(4)-\mathrm{Dy}(2)-\mathrm{N}(4)$ & $157.3(4)$ \\
\hline $\mathrm{O}(8)-\mathrm{Dy}(1)-\mathrm{O}(9)$ & $82.8(3)$ & $\mathrm{N}(5)-\mathrm{Dy}(2)-\mathrm{N}(4)$ & $64.6(4)$ \\
\hline $\mathrm{O}(7)-\mathrm{Dy}(1)-\mathrm{N}(6)$ & $73.7(4)$ & $\mathrm{N}(3)-\mathrm{Dy}(2)-\mathrm{N}(4)$ & $72.9(4)$ \\
\hline $\mathrm{O}(4)-\mathrm{Dy}(1)-\mathrm{N}(6)$ & $109.4(4)$ & $\mathrm{O}(6)-\mathrm{Dy}(2)-\mathrm{N}(2)$ & $147.4(3)$ \\
\hline $\mathrm{O}(2)-\mathrm{Dy}(1)-\mathrm{N}(6)$ & $146.1(4)$ & $\mathrm{O}(9)-\mathrm{Dy}(2)-\mathrm{N}(2)$ & $105.9(4)$ \\
\hline $\mathrm{O}(1)-\mathrm{Dy}(1)-\mathrm{N}(6)$ & $78.1(4)$ & $\mathrm{O}(5)-\mathrm{Dy}(2)-\mathrm{N}(2)$ & $136.0(4)$ \\
\hline $\mathrm{O}(8)-\mathrm{Dy}(1)-\mathrm{N}(6)$ & $138.3(4)$ & $\mathrm{O}(4)-\mathrm{Dy}(2)-\mathrm{N}(2)$ & $73.9(3)$ \\
\hline $\mathrm{O}(9)-\mathrm{Dy}(1)-\mathrm{N}(6)$ & $69.5(4)$ & $\mathrm{N}(5)-\mathrm{Dy}(2)-\mathrm{N}(2)$ & $69.8(4)$ \\
\hline $\mathrm{O}(7)-\mathrm{Dy}(1)-\mathrm{N}(1)$ & $144.1(4)$ & $\mathrm{N}(3)-\mathrm{Dy}(2)-\mathrm{N}(2)$ & $65.3(4)$ \\
\hline $\mathrm{O}(4)-\mathrm{Dy}(1)-\mathrm{N}(1)$ & $67.5(4)$ & $\mathrm{N}(4)-\mathrm{Dy}(2)-\mathrm{N}(2)$ & $86.8(4)$ \\
\hline $\mathrm{O}(2)-\mathrm{Dy}(1)-\mathrm{N}(1)$ & $77.0(4)$ & $\mathrm{Dy}(2)-\mathrm{O}(9)-\mathrm{Dy}(1)$ & $113.5(4)$ \\
\hline $\mathrm{Dy}(1)-\mathrm{O}(4)-\mathrm{Dy}(2)$ & $115.9(4)$ & & \\
\hline \multicolumn{4}{|l|}{ Compound $\mathbf{3}$} \\
\hline $\mathrm{Dy}(1)-\mathrm{O}(5)$ & $2.230(5)$ & $\mathrm{O}(5)-\mathrm{Dy}(1)-\mathrm{N}(4)$ & $132.89(17)$ \\
\hline Dy(1)-O(3) & $2.247(5)$ & $\mathrm{O}(3)-\mathrm{Dy}(1)-\mathrm{N}(4)$ & $74.94(18)$ \\
\hline $\mathrm{Dy}(1)-\mathrm{O}(1)$ & $2.343(5)$ & $\mathrm{O}(1)-\mathrm{Dy}(1)-\mathrm{N}(4)$ & $71.25(18)$ \\
\hline $\mathrm{Dy}(1)-\mathrm{O}(2)$ & $2.346(5)$ & $\mathrm{O}(2)-\mathrm{Dy}(1)-\mathrm{N}(4)$ & $105.64(18)$ \\
\hline $\operatorname{Dy}(1)-\mathrm{N}(1)$ & $2.560(6)$ & $\mathrm{N}(1)-\mathrm{Dy}(1)-\mathrm{N}(4)$ & $148.11(19)$ \\
\hline Dy $(1)-N(4)$ & $2.565(5)$ & $\mathrm{O}(5)-\mathrm{Dy}(1)-\mathrm{N}(3)$ & $74.73(17)$ \\
\hline $\mathrm{Dy}(1)-\mathrm{N}(3)$ & $2.629(5)$ & $\mathrm{O}(3)-\mathrm{Dy}(1)-\mathrm{N}(3)$ & $123.23(17)$ \\
\hline $\mathrm{Dy}(1)-\mathrm{N}(2)$ & $2.632(6)$ & $\mathrm{O}(1)-\mathrm{Dy}(1)-\mathrm{N}(3)$ & $79.04(17)$ \\
\hline $\mathrm{O}(5)-\mathrm{Dy}(1)-\mathrm{O}(3)$ & $151.00(18)$ & $\mathrm{O}(2)-\mathrm{Dy}(1)-\mathrm{N}(3)$ & $149.79(18)$ \\
\hline $\mathrm{O}(5)-\mathrm{Dy}(1)-\mathrm{O}(1)$ & $80.49(17)$ & $\mathrm{N}(1)-\mathrm{Dy}(1)-\mathrm{N}(3)$ & $125.13(18)$ \\
\hline $\mathrm{O}(3)-\mathrm{Dy}(1)-\mathrm{O}(1)$ & $122.78(16)$ & $\mathrm{N}(4)-\mathrm{Dy}(1)-\mathrm{N}(3)$ & $63.60(16)$ \\
\hline $\mathrm{O}(5)-\mathrm{Dy}(1)-\mathrm{O}(2)$ & $99.26(18)$ & $\mathrm{O}(5)-\mathrm{Dy}(1)-\mathrm{N}(2)$ & $93.00(18)$ \\
\hline $\mathrm{O}(3)-\mathrm{Dy}(1)-\mathrm{O}(2)$ & $75.89(18)$ & $\mathrm{O}(3)-\mathrm{Dy}(1)-\mathrm{N}(2)$ & $75.14(18)$ \\
\hline $\mathrm{O}(1)-\mathrm{Dy}(1)-\mathrm{O}(2)$ & $70.75(17)$ & $\mathrm{O}(1)-\mathrm{Dy}(1)-\mathrm{N}(2)$ & $149.19(17)$ \\
\hline $\mathrm{O}(5)-\mathrm{Dy}(1)-\mathrm{N}(1)$ & $74.92(18)$ & $\mathrm{O}(2)-\mathrm{Dy}(1)-\mathrm{N}(2)$ & $139.99(18)$ \\
\hline $\mathrm{O}(3)-\mathrm{Dy}(1)-\mathrm{N}(1)$ & $76.08(17)$ & $\mathrm{N}(1)-\mathrm{Dy}(1)-\mathrm{N}(2)$ & $66.98(18)$ \\
\hline $\mathrm{O}(1)-\mathrm{Dy}(1)-\mathrm{N}(1)$ & $137.59(18)$ & $\mathrm{N}(4)-\mathrm{Dy}(1)-\mathrm{N}(2)$ & $92.77(18)$ \\
\hline $\mathrm{O}(2)-\mathrm{Dy}(1)-\mathrm{N}(1)$ & $79.68(19)$ & $\mathrm{N}(3)-\mathrm{Dy}(1)-\mathrm{N}(2)$ & $70.22(18)$ \\
\hline \multicolumn{4}{|l|}{ Compound 4} \\
\hline Dy(2)-Dy(1) & $3.8223(12)$ & $\mathrm{O}(9)-\mathrm{Dy}(2)-\mathrm{Dy}(1)$ & $33.5(2)$ \\
\hline $\mathrm{Dy}(2)-\mathrm{N}(2)$ & $2.531(12)$ & $\mathrm{O}(9)-\mathrm{Dy}(2)-\mathrm{N}(2)$ & $150.5(4)$ \\
\hline
\end{tabular}




\begin{tabular}{|c|c|c|c|}
\hline $\mathrm{Dy}(2)-\mathrm{N}(3)$ & $2.577(11)$ & $\mathrm{O}(9)-\mathrm{Dy}(2)-\mathrm{N}(3)$ & $106.8(3)$ \\
\hline $\mathrm{Dy}(2)-\mathrm{N}(4)$ & $2.566(12)$ & $\mathrm{O}(9)-\mathrm{Dy}(2)-\mathrm{N}(4)$ & $134.5(4)$ \\
\hline Dy $(2)-N(5)$ & $2.562(11)$ & $\mathrm{O}(9)-\mathrm{Dy}(2)-\mathrm{N}(5)$ & $77.9(3)$ \\
\hline $\mathrm{Dy}(2)-\mathrm{O}(5)$ & $2.313(9)$ & $\mathrm{O}(9)-\mathrm{Dy}(2)-\mathrm{O}(5)$ & $81.7(3)$ \\
\hline $\mathrm{Dy}(2)-\mathrm{O}(6)$ & $2.279(9)$ & $\mathrm{O}(9)-\mathrm{Dy}(2)-\mathrm{O}(7)$ & $65.7(3)$ \\
\hline $\mathrm{Dy}(2)-\mathrm{O}(7)$ & $2.325(9)$ & $\mathrm{N}(1)-\mathrm{Dy}(1)-\mathrm{Dy}(2)$ & $87.3(3)$ \\
\hline $\mathrm{Dy}(2)-\mathrm{O}(9)$ & $2.282(9)$ & $\mathrm{N}(6)-\mathrm{Dy}(1)-\mathrm{Dy}(2)$ & $84.4(3)$ \\
\hline Dy $(1)-\mathrm{N}(1)$ & $2.585(13)$ & $\mathrm{N}(6)-\mathrm{Dy}(1)-\mathrm{N}(1)$ & $77.1(4)$ \\
\hline Dy $(1)-N(6)$ & $2.550(13)$ & $\mathrm{O}(1)-\mathrm{Dy}(1)-\mathrm{Dy}(2)$ & $112.0(2)$ \\
\hline $\mathrm{Dy}(1)-\mathrm{O}(1)$ & $2.327(10)$ & $\mathrm{O}(1)-\mathrm{Dy}(1)-\mathrm{N}(1)$ & $73.7(4)$ \\
\hline $\mathrm{Dy}(1)-\mathrm{O}(2)$ & $2.279(10)$ & $\mathrm{O}(1)-\mathrm{Dy}(1)-\mathrm{N}(6)$ & $145.4(4)$ \\
\hline $\mathrm{Dy}(1)-\mathrm{O}(3)$ & $2.304(10)$ & $\mathrm{O}(2)-\mathrm{Dy}(1)-\mathrm{Dy}(2)$ & $162.6(3)$ \\
\hline $\mathrm{Dy}(1)-\mathrm{O}(4)$ & $2.314(10)$ & $\mathrm{O}(2)-\mathrm{Dy}(1)-\mathrm{N}(1)$ & $77.7(4)$ \\
\hline $\mathrm{Dy}(1)-\mathrm{O}(7)$ & $2.247(8)$ & $\mathrm{O}(2)-\mathrm{Dy}(1)-\mathrm{N}(6)$ & $83.7(4)$ \\
\hline $\mathrm{Dy}(1)-\mathrm{O}(9)$ & $2.296(9)$ & $\mathrm{O}(2)-\mathrm{Dy}(1)-\mathrm{O}(1)$ & $72.4(3)$ \\
\hline $\mathrm{N}(2)-\mathrm{Dy}(2)-\mathrm{Dy}(1)$ & $117.0(3)$ & $\mathrm{O}(2)-\mathrm{Dy}(1)-\mathrm{O}(3)$ & $75.0(4)$ \\
\hline $\mathrm{N}(2)-\mathrm{Dy}(2)-\mathrm{N}(3)$ & $65.0(4)$ & $\mathrm{O}(2)-\mathrm{Dy}(1)-\mathrm{O}(4)$ & $109.7(4)$ \\
\hline $\mathrm{N}(2)-\mathrm{Dy}(2)-\mathrm{N}(4)$ & $74.7(4)$ & $\mathrm{O}(2)-\mathrm{Dy}(1)-\mathrm{O}(9)$ & $147.7(3)$ \\
\hline $\mathrm{N}(2)-\mathrm{Dy}(2)-\mathrm{N}(5)$ & $121.3(3)$ & $\mathrm{O}(3)-\mathrm{Dy}(1)-\mathrm{Dy}(2)$ & $113.3(3)$ \\
\hline $\mathrm{N}(3)-\mathrm{Dy}(2)-\mathrm{Dy}(1)$ & $93.6(3)$ & $\mathrm{O}(3)-\mathrm{Dy}(1)-\mathrm{N}(1)$ & $141.2(4)$ \\
\hline $\mathrm{N}(4)-\mathrm{Dy}(2)-\mathrm{Dy}(1)$ & $166.8(3)$ & $\mathrm{O}(3)-\mathrm{Dy}(1)-\mathrm{N}(6)$ & $73.0(4)$ \\
\hline $\mathrm{N}(4)-\mathrm{Dy}(2)-\mathrm{N}(3)$ & $85.8(4)$ & $\mathrm{O}(3)-\mathrm{Dy}(1)-\mathrm{O}(1)$ & $121.9(4)$ \\
\hline $\mathrm{N}(5)-\mathrm{Dy}(2)-\mathrm{Dy}(1)$ & $102.3(3)$ & $\mathrm{O}(3)-\mathrm{Dy}(1)-\mathrm{O}(4)$ & $72.9(4)$ \\
\hline $\mathrm{N}(5)-\mathrm{Dy}(2)-\mathrm{N}(3)$ & $70.9(3)$ & $\mathrm{O}(4)-\mathrm{Dy}(1)-\mathrm{Dy}(2)$ & $87.6(2)$ \\
\hline $\mathrm{N}(5)-\mathrm{Dy}(2)-\mathrm{N}(4)$ & $65.1(4)$ & $\mathrm{O}(4)-\mathrm{Dy}(1)-\mathrm{N}(1)$ & $143.4(4)$ \\
\hline $\mathrm{O}(5)-\mathrm{Dy}(2)-\mathrm{Dy}(1)$ & $108.6(2)$ & $\mathrm{O}(4)-\mathrm{Dy}(1)-\mathrm{N}(6)$ & $138.2(4)$ \\
\hline $\mathrm{O}(5)-\mathrm{Dy}(2)-\mathrm{N}(2)$ & $121.8(4)$ & $\mathrm{O}(4)-\mathrm{Dy}(1)-\mathrm{O}(1)$ & $74.8(3)$ \\
\hline $\mathrm{O}(5)-\mathrm{Dy}(2)-\mathrm{N}(3)$ & $145.7(3)$ & $\mathrm{O}(7)-\mathrm{Dy}(1)-\mathrm{Dy}(2)$ & $33.9(2)$ \\
\hline $\mathrm{O}(5)-\mathrm{Dy}(2)-\mathrm{N}(4)$ & $66.5(3)$ & $\mathrm{O}(7)-\mathrm{Dy}(1)-\mathrm{N}(1)$ & $68.9(3)$ \\
\hline $\mathrm{O}(5)-\mathrm{Dy}(2)-\mathrm{N}(5)$ & $78.8(3)$ & $\mathrm{O}(7)-\mathrm{Dy}(1)-\mathrm{N}(6)$ & $107.0(4)$ \\
\hline $\mathrm{O}(5)-\mathrm{Dy}(2)-\mathrm{O}(7)$ & $136.7(3)$ & $\mathrm{O}(7)-\mathrm{Dy}(1)-\mathrm{O}(1)$ & $79.4(3)$ \\
\hline $\mathrm{O}(6)-\mathrm{Dy}(2)-\mathrm{Dy}(1)$ & $82.9(3)$ & $\mathrm{O}(7)-\mathrm{Dy}(1)-\mathrm{O}(2)$ & $141.1(3)$ \\
\hline $\mathrm{O}(6)-\mathrm{Dy}(2)-\mathrm{N}(2)$ & $79.5(4)$ & $\mathrm{O}(7)-\mathrm{Dy}(1)-\mathrm{O}(3)$ & $143.8(3)$ \\
\hline $\mathrm{O}(6)-\mathrm{Dy}(2)-\mathrm{N}(3)$ & $138.2(3)$ & $\mathrm{O}(7)-\mathrm{Dy}(1)-\mathrm{O}(4)$ & $87.4(3)$ \\
\hline $\mathrm{O}(6)-\mathrm{Dy}(2)-\mathrm{N}(4)$ & $106.2(4)$ & $\mathrm{O}(7)-\mathrm{Dy}(1)-\mathrm{O}(9)$ & $66.8(3)$ \\
\hline $\mathrm{O}(6)-\mathrm{Dy}(2)-\mathrm{N}(5)$ & $150.6(4)$ & $\mathrm{O}(9)-\mathrm{Dy}(1)-\mathrm{Dy}(2)$ & $33.3(2)$ \\
\hline $\mathrm{O}(6)-\mathrm{Dy}(2)-\mathrm{O}(5)$ & $72.1(3)$ & $\mathrm{O}(9)-\mathrm{Dy}(1)-\mathrm{N}(1)$ & $111.3(4)$ \\
\hline $\mathrm{O}(6)-\mathrm{Dy}(2)-\mathrm{O}(7)$ & $81.6(3)$ & $\mathrm{O}(9)-\mathrm{Dy}(1)-\mathrm{N}(6)$ & $69.2(3)$ \\
\hline $\mathrm{O}(6)-\mathrm{Dy}(2)-\mathrm{O}(9)$ & $93.0(3)$ & $\mathrm{O}(9)-\mathrm{Dy}(1)-\mathrm{O}(1)$ & $139.5(3)$ \\
\hline $\mathrm{O}(7)-\mathrm{Dy}(2)-\mathrm{Dy}(1)$ & $32.6(2)$ & $\mathrm{O}(9)-\mathrm{Dy}(1)-\mathrm{O}(3)$ & $80.4(3)$ \\
\hline $\mathrm{O}(7)-\mathrm{Dy}(2)-\mathrm{N}(2)$ & $84.9(3)$ & $\mathrm{O}(9)-\mathrm{Dy}(1)-\mathrm{O}(4)$ & $82.0(3)$ \\
\hline $\mathrm{O}(7)-\mathrm{Dy}(2)-\mathrm{N}(3)$ & $74.1(3)$ & $\mathrm{O}(8)-\mathrm{N}(1)-\mathrm{Dy}(1)$ & $115.0(9)$ \\
\hline $\mathrm{O}(7)-\mathrm{Dy}(2)-\mathrm{N}(4)$ & $156.1(3)$ & $\mathrm{Dy}(1)-\mathrm{O}(7)-\mathrm{Dy}(2)$ & $113.5(3)$ \\
\hline
\end{tabular}




\begin{tabular}{|l|l|l|l|l}
$\mathrm{O}(7)-\mathrm{Dy}(2)-\mathrm{N}(5)$ & $118.2(3)$ & $\mathrm{Dy}(2)-\mathrm{O}(9)-\mathrm{Dy}(1)$ & $113.2(4)$ \\
\hline
\end{tabular}

Table S2 Dy (III) ion geometry analysis by SHAPE 2.1 software.

\begin{tabular}{|c|c|c|c|}
\hline & \multirow{2}{*}{ ABOXIY } & \multicolumn{2}{c|}{ ABOXIY } \\
\cline { 3 - 4 } Configuration & $\mathbf{1}$ & Dy1 & Dy2 \\
\hline Octagon $\left(D_{8 \mathrm{~h}}\right)$ & & 30.678 & 32.574 \\
\hline Heptagonal pyramid $\left(C_{7 \mathrm{v}}\right)$ & 31.959 & 22.625 & 22.848 \\
\hline Hexagonal bipyramid $\left(D_{6 \mathrm{~h}}\right)$ & 21.756 & 16.022 & 12.414 \\
\hline Cube $\left(O_{\mathrm{h}}\right)$ & 12.441 & 9.215 & 5.454 \\
\hline Square antiprism $\left(D_{4 \mathrm{~d}}\right)$ & 8.180 & 0.714 & 2.387 \\
\hline Triangular dodecahedron $\left(D_{2 \mathrm{~d}}\right)$ & 2.288 & 2.449 & 1.185 \\
\hline Johnson gyrobifastigium $\mathrm{J} 26\left(D_{2 \mathrm{~d}}\right)$ & 1.332 & 15.753 & 15.315 \\
\hline Johnson elongated triangular bipyramid $\mathrm{J} 14\left(D_{3 \mathrm{~h}}\right)$ & 12.432 & 27.101 & 27.332 \\
\hline Biaugmented trigonal prism $\mathrm{J} 50\left(C_{2 \mathrm{v}}\right)$ & 26.343 & 3.244 & 3.952 \\
\hline Biaugmented trigonal prism $\left(C_{2 \mathrm{v}}\right)$ & 1.991 & 2.505 & 3.277 \\
\hline Snub diphenoid J84 $\left(D_{2 \mathrm{~d}}\right)$ & 1.957 & 5.562 & 5.026 \\
\hline Triakis tetrahedron $\left(T_{\mathrm{d}}\right)$ & 4.441 & 10.017 & 6.331 \\
\hline Elongated trigonal bipyramid $\left(D_{3 \mathrm{~h}}\right)$ & 8.926 & 22.484 & 23.935 \\
\hline
\end{tabular}

\begin{tabular}{|c|c|c|c|}
\hline & \multirow{2}{*}{ ABOXIY } & \multicolumn{2}{c|}{ ABOXIY } \\
\cline { 2 - 4 } Configuration & $\mathbf{3}$ & Dy1 & Dy2 \\
\cline { 3 - 4 } & & 31.214 & 31.169 \\
\hline Octagon $\left(D_{8 \mathrm{~h}}\right)$ & 33.061 & 22.043 & 22.170 \\
\hline Heptagonal pyramid $\left(C_{7 \mathrm{v}}\right)$ & 21.626 & 16.033 & 13.404 \\
\hline Hexagonal bipyramid $\left(D_{6 \mathrm{~h}}\right)$ & 12.868 & 9.250 & 6.347 \\
\hline Cube $\left(O_{\mathrm{h}}\right)$ & 7.199 & 0.833 & 1.845 \\
\hline Square antiprism $\left(D_{4 \mathrm{~d}}\right)$ & 2.820 & 1.831 & 1.182 \\
\hline Triangular dodecahedron $\left(D_{2 \mathrm{~d}}\right)$ & 0.723 & 16.605 & 15.832 \\
\hline Johnson gyrobifastigium $\mathrm{J} 26\left(D_{2 \mathrm{~d}}\right)$ & 14.629 & 27.274 & 27.670 \\
\hline Johnson elongated triangular bipyramid J14 $\left(D_{3 \mathrm{~h}}\right)$ & 27.756 & 2.865 & 3.592 \\
\hline Biaugmented trigonal prism $\mathrm{J} 50\left(C_{2 \mathrm{v}}\right)$ & 2.657 & 2.220 & 2.963 \\
\hline Biaugmented trigonal prism $\left(C_{2 \mathrm{v}}\right)$ & 2.661 & 4.635 & 4.678 \\
\hline Snub diphenoid J84 $\left(D_{2 \mathrm{~d}}\right)$ & 3.903 & 10.099 & 7.218 \\
\hline Triakis tetrahedron $\left(T_{\mathrm{d}}\right)$ & 7.993 & 23.381 & 24.364 \\
\hline Elongated trigonal bipyramid $\left(D_{3 \mathrm{~h}}\right)$ & 24.896 & & \\
\hline
\end{tabular}

S H A P E v2.1 Continuous Shape Measures calculation

(c) 2013 Electronic Structure Group, Universitat de Barcelona

Contact: llunell@ub.edu 
Dy structures in 1

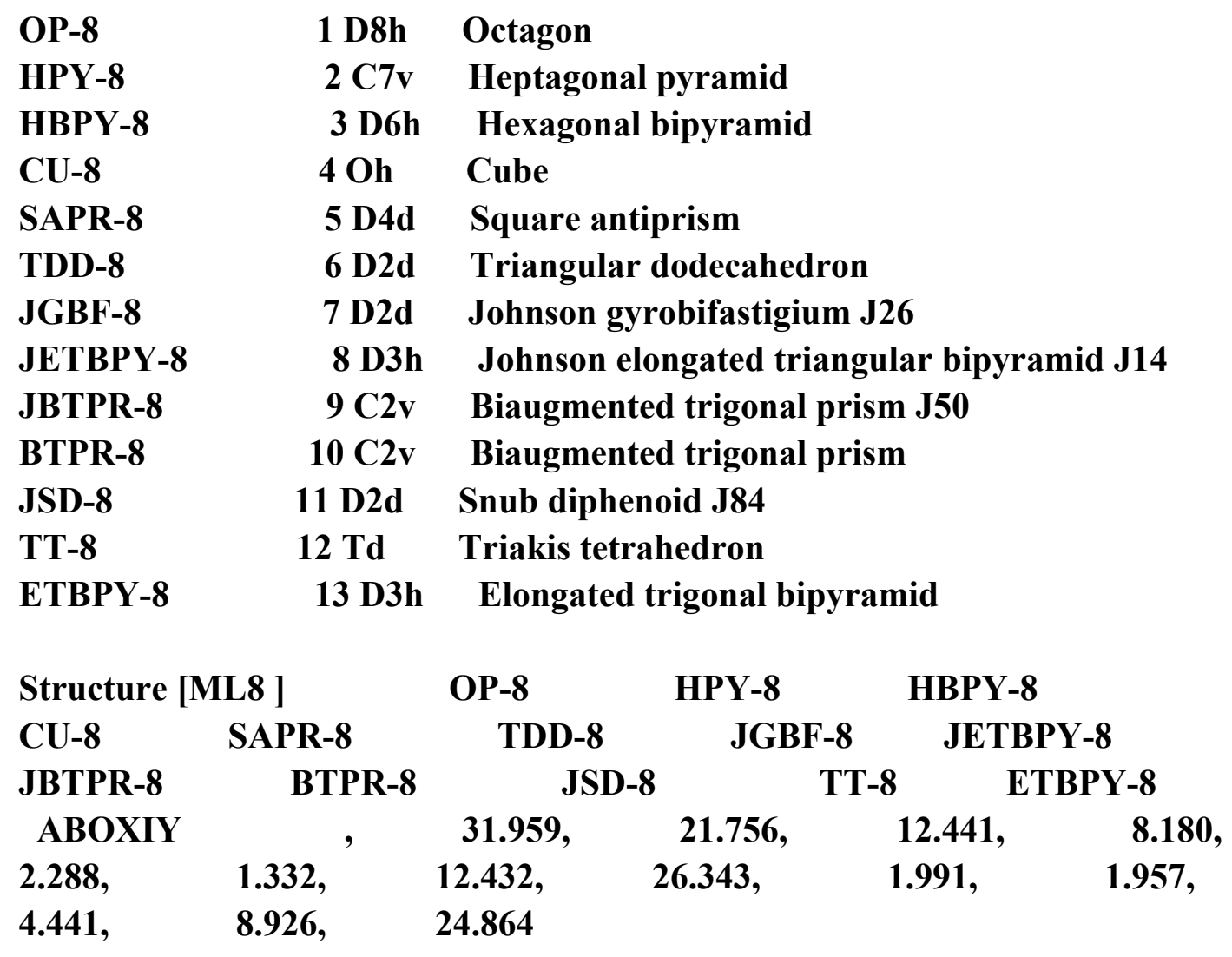

S H A P E v2.1 Continuous Shape Measures calculation

(c) 2013 Electronic Structure Group, Universitat de Barcelona

Contact: llunell@ub.edu

Dy structure in 2 (Dy1)

\begin{tabular}{|c|c|c|}
\hline OP-8 & $1 \mathrm{D8h}$ & Octagon \\
\hline HPY-8 & $2 \mathbf{C 7 v}$ & Heptagonal pyramid \\
\hline HBPY-8 & 3 D6h & Hexagonal bipyramid \\
\hline CU-8 & $4 \mathrm{Oh}$ & Cube \\
\hline SAPR-8 & 5 D4d & Square antiprism \\
\hline TDD-8 & 6 D2d & Triangular dodecahedron \\
\hline JGBF-8 & 7 D2d & Johnson gyrobifastigium J26 \\
\hline JETBPY-8 & 8 D3h & Johnson elongated triangular bipyramid J14 \\
\hline JBTPR-8 & $9 \mathrm{C} 2 \mathrm{v}$ & Biaugmented trigonal prism $\mathbf{J 5 0}$ \\
\hline BTPR-8 & $10 \mathrm{C} 2 \mathrm{v}$ & Biaugmented trigonal prism \\
\hline JSD-8 & $11 \mathrm{D} 2 d$ & Snub diphenoid J84 \\
\hline
\end{tabular}


$\begin{array}{lcc}\text { TT-8 } & 12 \text { Td } & \text { Triakis tetrahedron } \\ \text { ETBPY-8 } & 13 \mathrm{D3h} & \text { Elongated trigonal bipyramid }\end{array}$



S H A P E v2.1 Continuous Shape Measures calculation

(c) 2013 Electronic Structure Group, Universitat de Barcelona

Contact: llunell@ub.edu

Dy structures in 2 (Dy2)

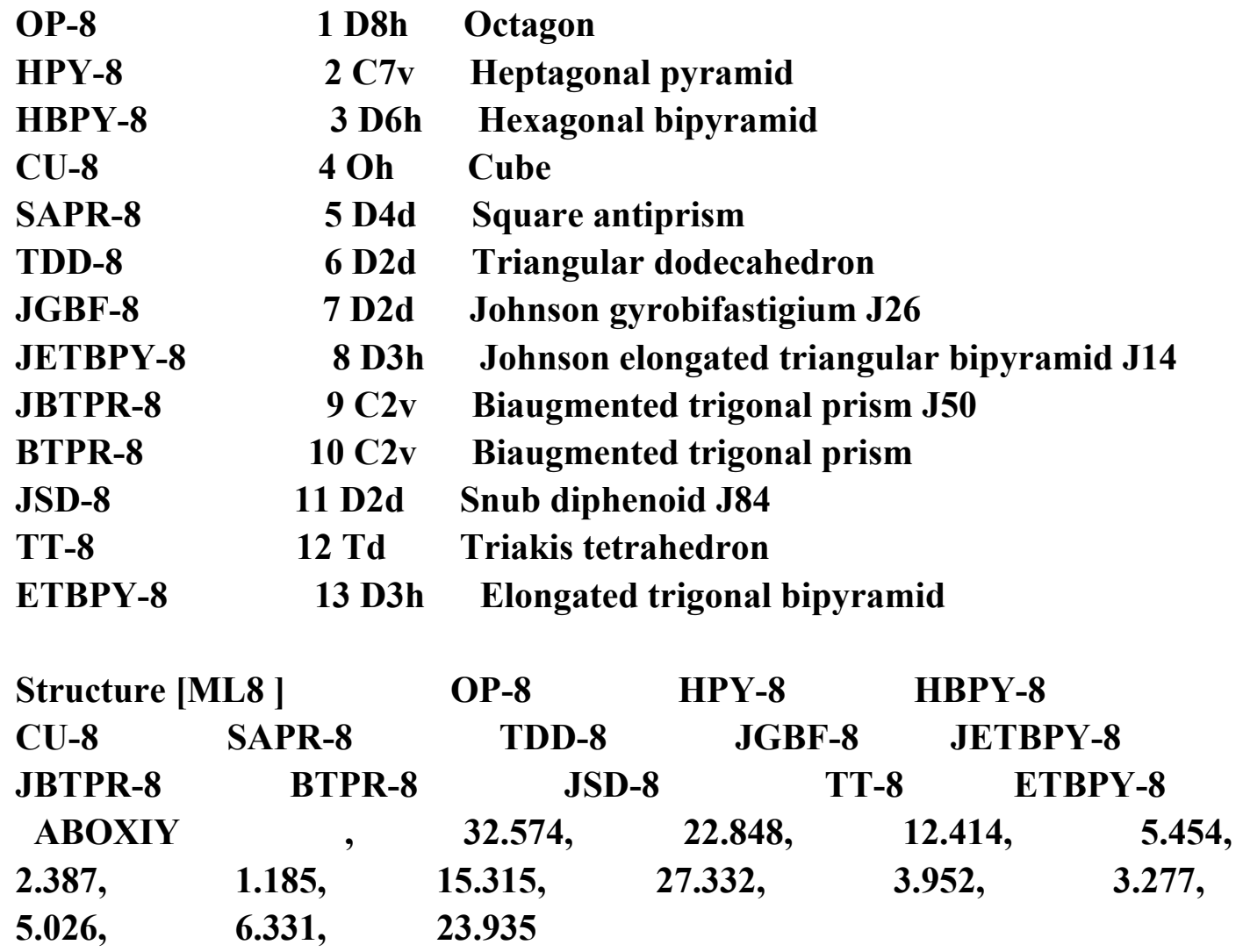

S H A P E v2.1 Continuous Shape Measures calculation

(c) 2013 Electronic Structure Group, Universitat de Barcelona

Contact: llunell@ub.edu 
Dy structures in 3

\begin{tabular}{|c|c|c|c|c|}
\hline OP-8 & $1 \mathrm{D8h}$ & \multicolumn{3}{|l|}{ Octagon } \\
\hline HPY-8 & $2 \mathbf{C} 7 \mathbf{v}$ & \multicolumn{3}{|l|}{ Heptagonal pyramid } \\
\hline HBPY-8 & 3 D6h & \multicolumn{3}{|l|}{ Hexagonal bipyramid } \\
\hline CU-8 & $4 \mathrm{Oh}$ & \multicolumn{3}{|l|}{ Cube } \\
\hline SAPR-8 & 5 D4d & \multicolumn{3}{|l|}{ Square antiprism } \\
\hline TDD-8 & 6 D2d & \multicolumn{3}{|c|}{ Triangular dodecahedron } \\
\hline JGBF-8 & 7 D2d & \multicolumn{3}{|c|}{ Johnson gyrobifastigium J26 } \\
\hline JETBPY-8 & 8 D3h & \multicolumn{3}{|c|}{ Johnson elongated triangular bipyramid J14 } \\
\hline JBTPR-8 & $9 \mathrm{C} 2 \mathrm{v}$ & \multicolumn{3}{|c|}{ Biaugmented trigonal prism $\mathbf{J 5 0}$} \\
\hline BTPR-8 & $10 \mathrm{C} 2 \mathrm{v}$ & \multicolumn{3}{|c|}{ Biaugmented trigonal prism } \\
\hline JSD-8 & $11 \mathrm{D} 2 \mathrm{~d}$ & \multicolumn{3}{|l|}{ Snub diphenoid J84 } \\
\hline TT-8 & 12 Td & \multicolumn{3}{|l|}{ Triakis tetrahedron } \\
\hline ETBPY-8 & $13 \mathrm{D3h}$ & \multicolumn{3}{|c|}{ Elongated trigonal bipyramid } \\
\hline \multicolumn{2}{|c|}{ Structure [ML8 ] } & OP-8 & HPY-8 & HBPY-8 \\
\hline CU-8 & SAPR-8 & TDD-8 & JGBF-8 & JETBPY-8 \\
\hline JBTPR-8 & BTPR-8 & JSD-8 & TT-8 & PY-8 \\
\hline ABOXIY & , & 21.626, & 12.868 & 7.199, \\
\hline $\begin{array}{l}2.820 \\
3.903\end{array}$ & $\begin{array}{c}\text { 0.723, } \\
\text { 7.993, }\end{array}$ & $\begin{array}{c}14.629 \\
24.896\end{array}$ & 2.657, & 2.661, \\
\hline
\end{tabular}

S H A P E v2.1 Continuous Shape Measures calculation

(c) 2013 Electronic Structure Group, Universitat de Barcelona

Contact: llunell@ub.edu

Dy structures in 4 (Dy1)

$\begin{array}{lcl}\text { OP-8 } & 1 \mathrm{D8h} & \text { Octagon } \\ \text { HPY-8 } & 2 \mathrm{C7v} & \text { Heptagonal pyramid } \\ \text { HBPY-8 } & 3 \mathrm{D} 6 \mathrm{~h} & \text { Hexagonal bipyramid } \\ \text { CU-8 } & 4 \mathrm{Oh} & \text { Cube } \\ \text { SAPR-8 } & 5 \mathrm{D4d} & \text { Square antiprism } \\ \text { TDD-8 } & 6 \mathrm{D2d} & \text { Triangular dodecahedron } \\ \text { JGBF-8 } & 7 \mathrm{D} 2 \mathrm{~d} & \text { Johnson gyrobifastigium J26 } \\ \text { JETBPY-8 } & 8 \mathrm{D3h} & \text { Johnson elongated triangular bipyramid J14 } \\ \text { JBTPR-8 } & 9 \mathrm{C2v} & \text { Biaugmented trigonal prism J50 } \\ \text { BTPR-8 } & 10 \mathrm{C2v} & \text { Biaugmented trigonal prism }\end{array}$




$\begin{array}{lcc}\text { JSD-8 } & 11 \text { D2d } & \text { Snub diphenoid J84 } \\ \text { TT-8 } & 12 \text { Td } & \text { Triakis tetrahedron } \\ \text { ETBPY-8 } & 13 \text { D3h } & \text { Elongated trigonal bipyramid }\end{array}$

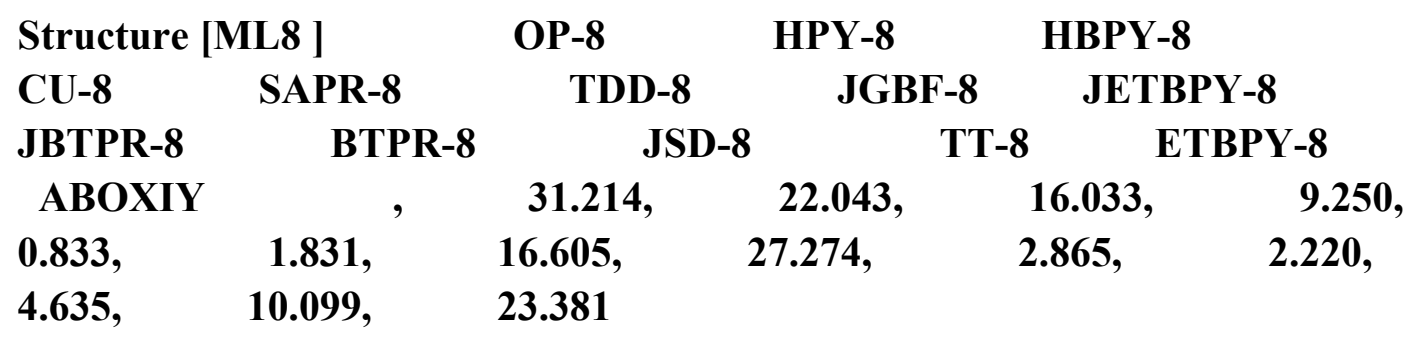

S H A P E $\quad$ v2.1 Continuous Shape Measures calculation

(c) 2013 Electronic Structure Group, Universitat de Barcelona

Contact: llunell@ub.edu

Dy structures in 4 (Dy2)

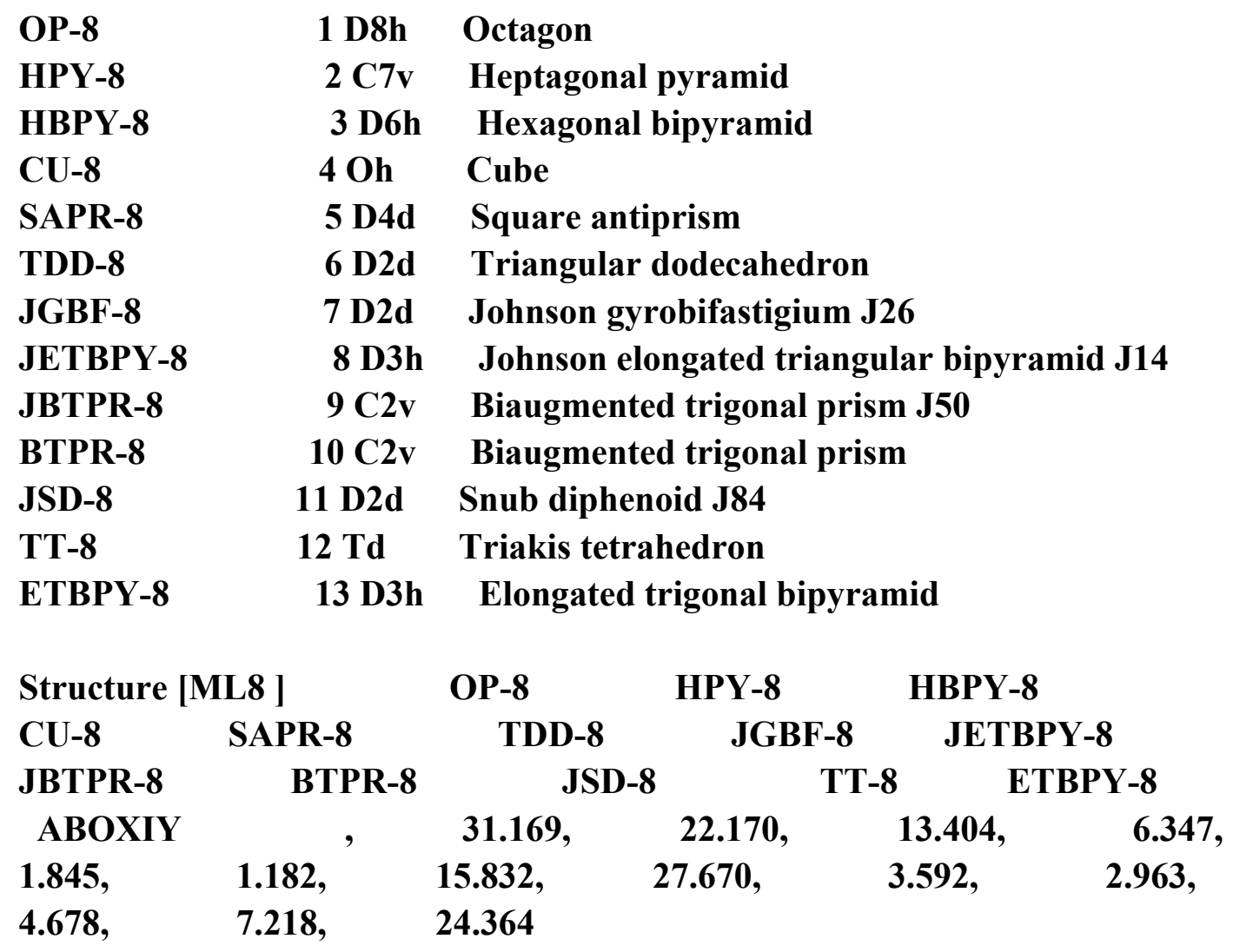




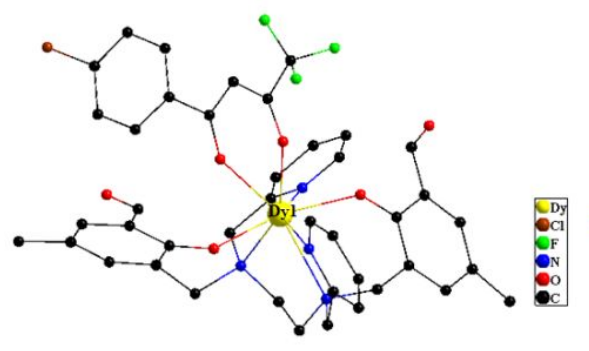

$\mathrm{NH}_{2} \mathrm{OH} \cdot \mathrm{HCl}$

$\mathrm{Na}_{2} \mathrm{CO}_{3}$

$\mathrm{CH}_{3} \mathrm{OH}, \mathrm{C}_{4} \mathrm{H}_{10} \mathrm{O}$, DMF

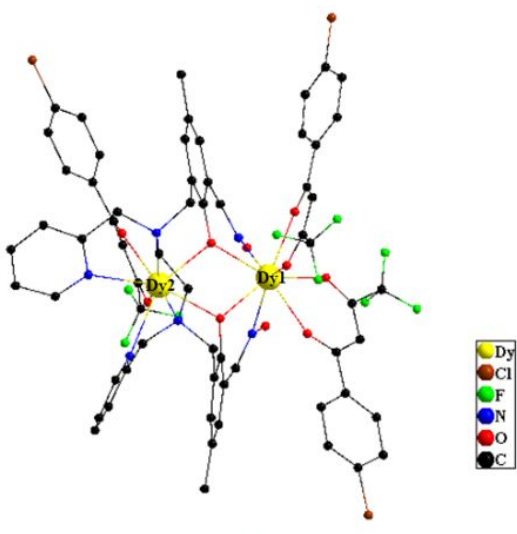

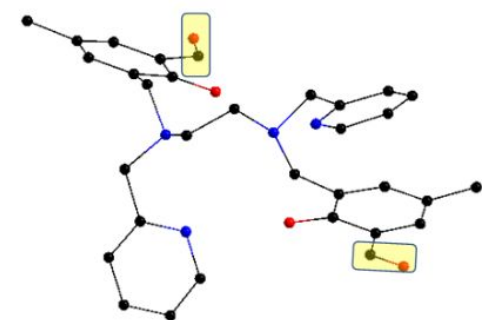

(a)
$30 \mathrm{~h}$ at room temperatu

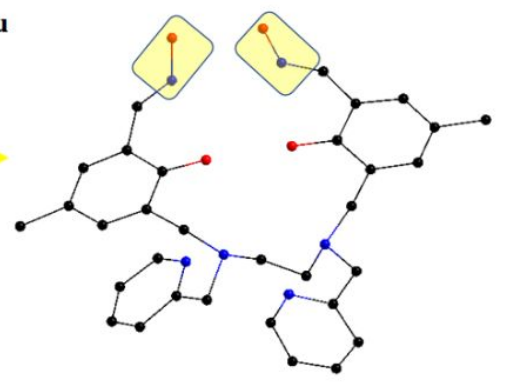

(b)

Figure S9. The crystal structures of compounds 3 (a) and 4 (b) showing general ligand configurations.

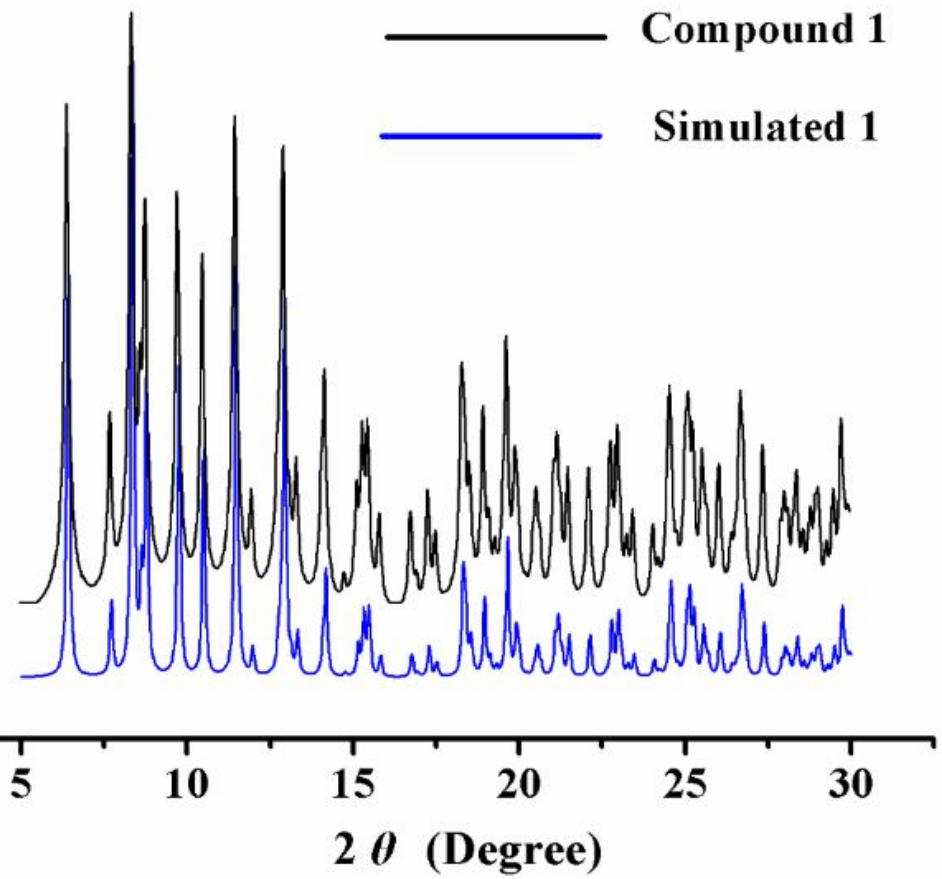

Figure S10. XRPD curves of 1. 


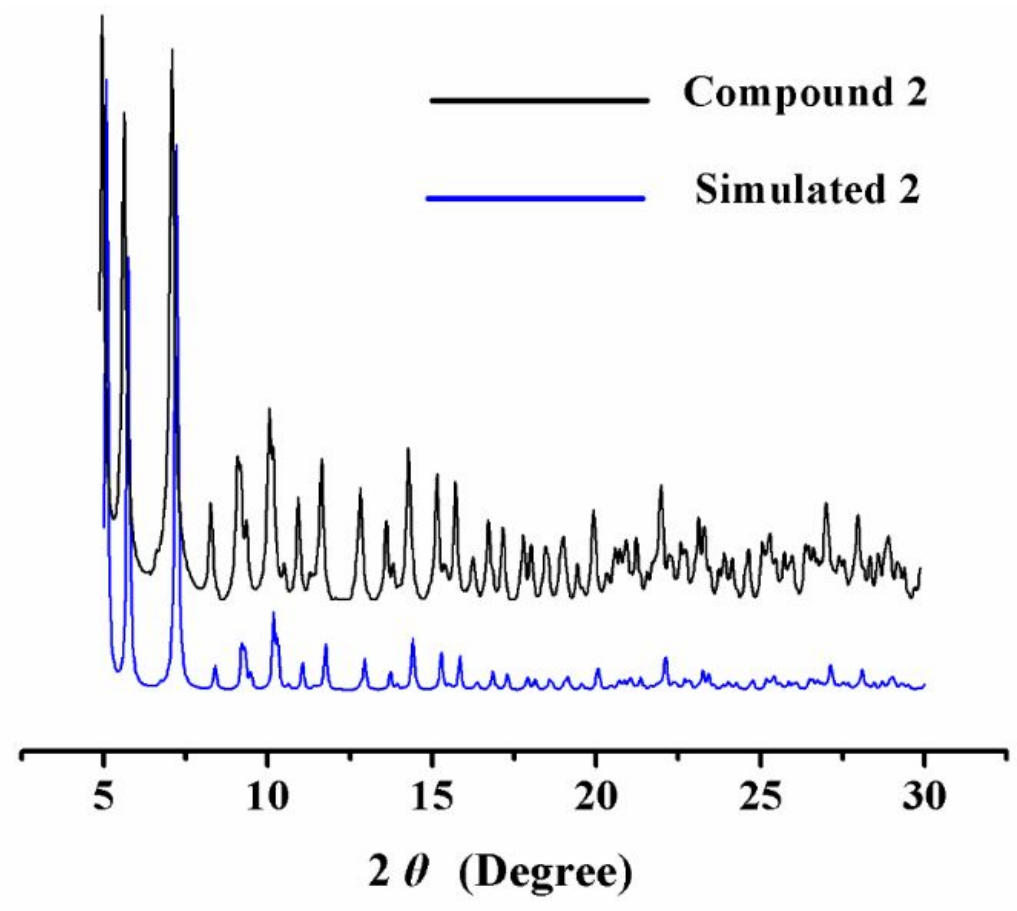

Figure S11. XRPD curves of 2.

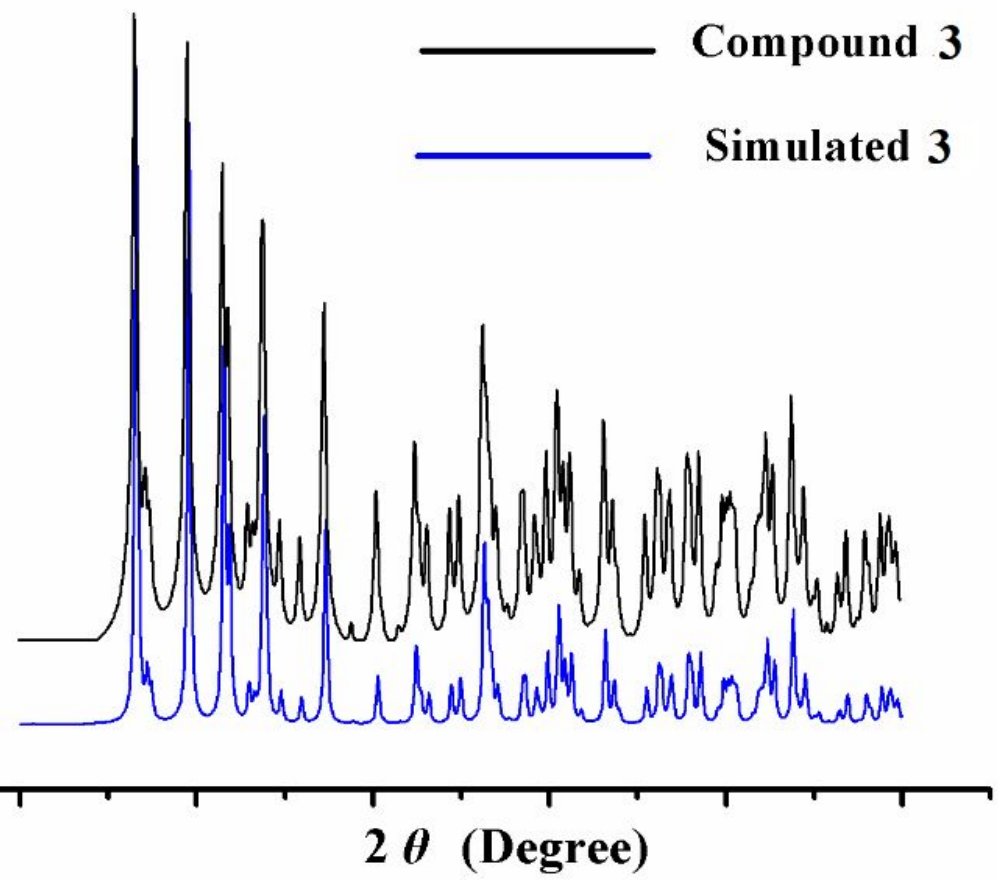

Figure S12. XRPD curves of 3. 


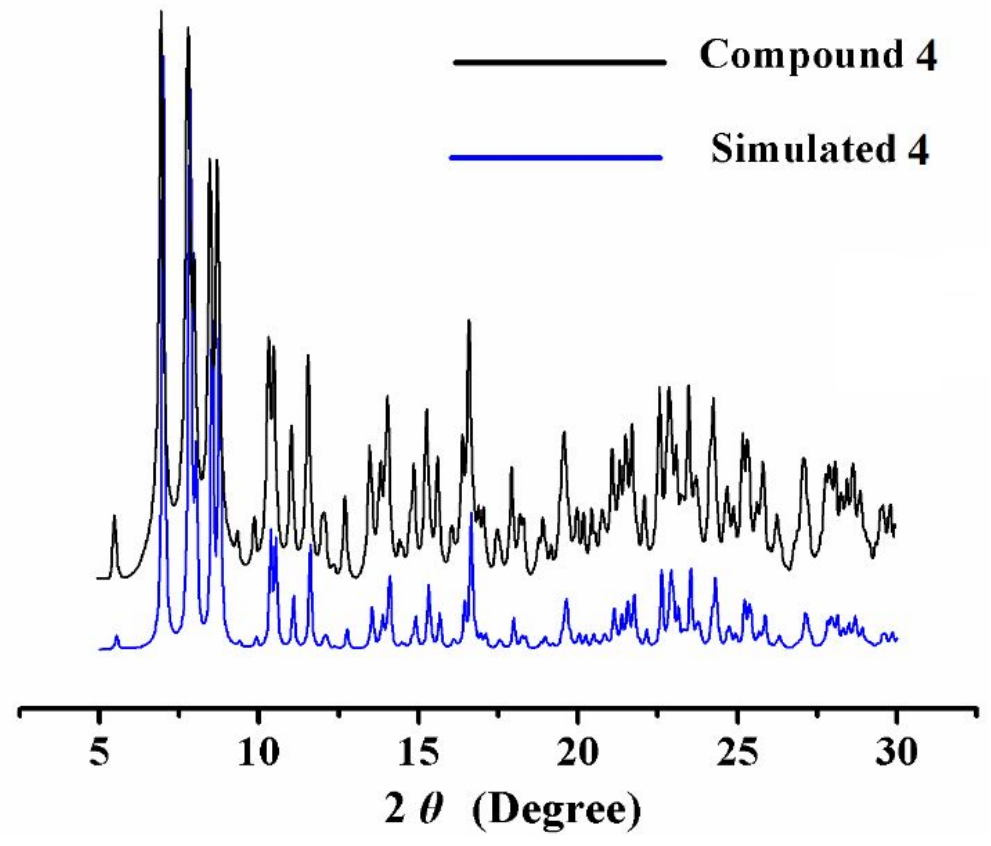

Figure S13. XRPD curves of 4.

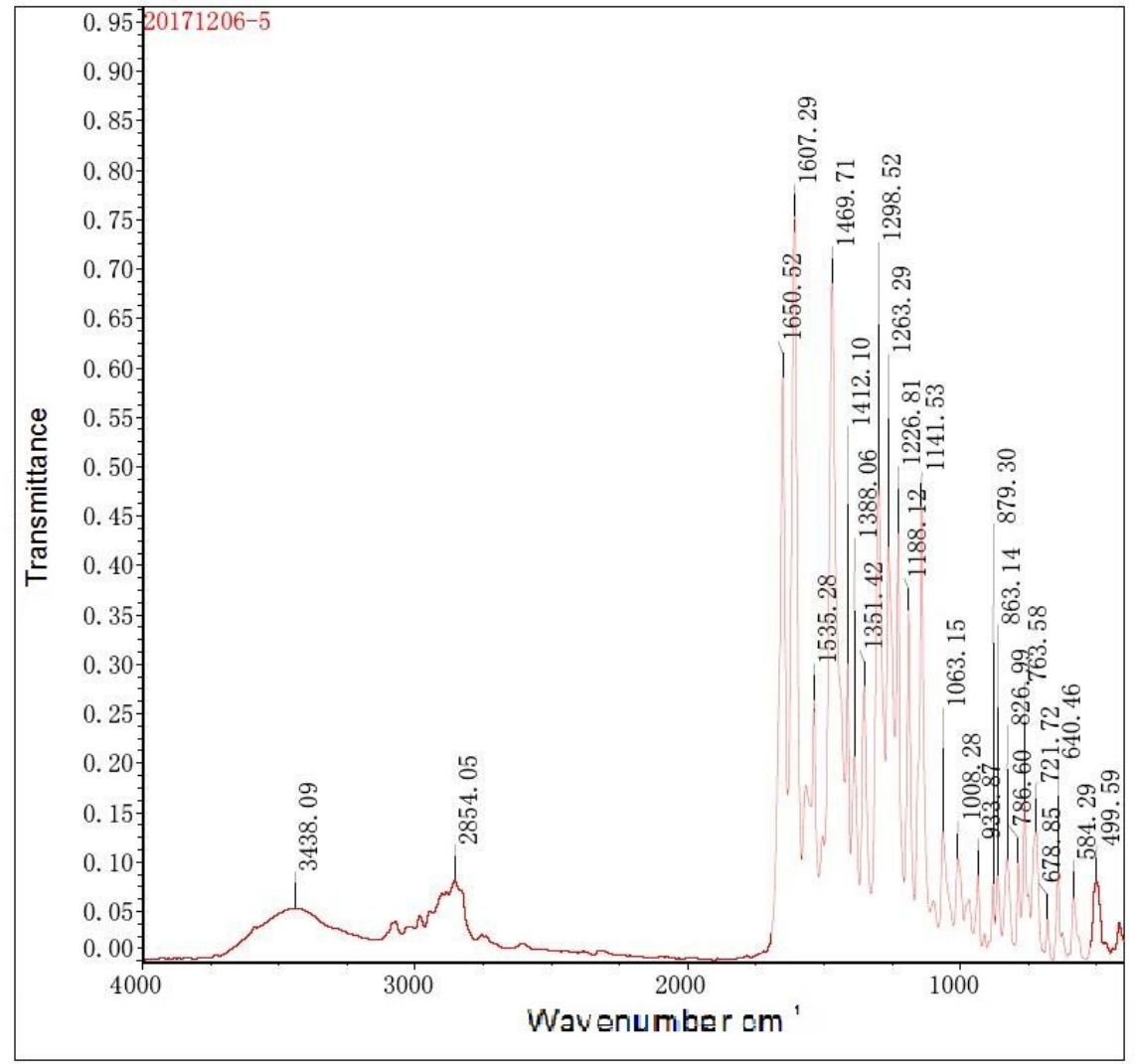

Figure S14. FTIR spectra of 1. 


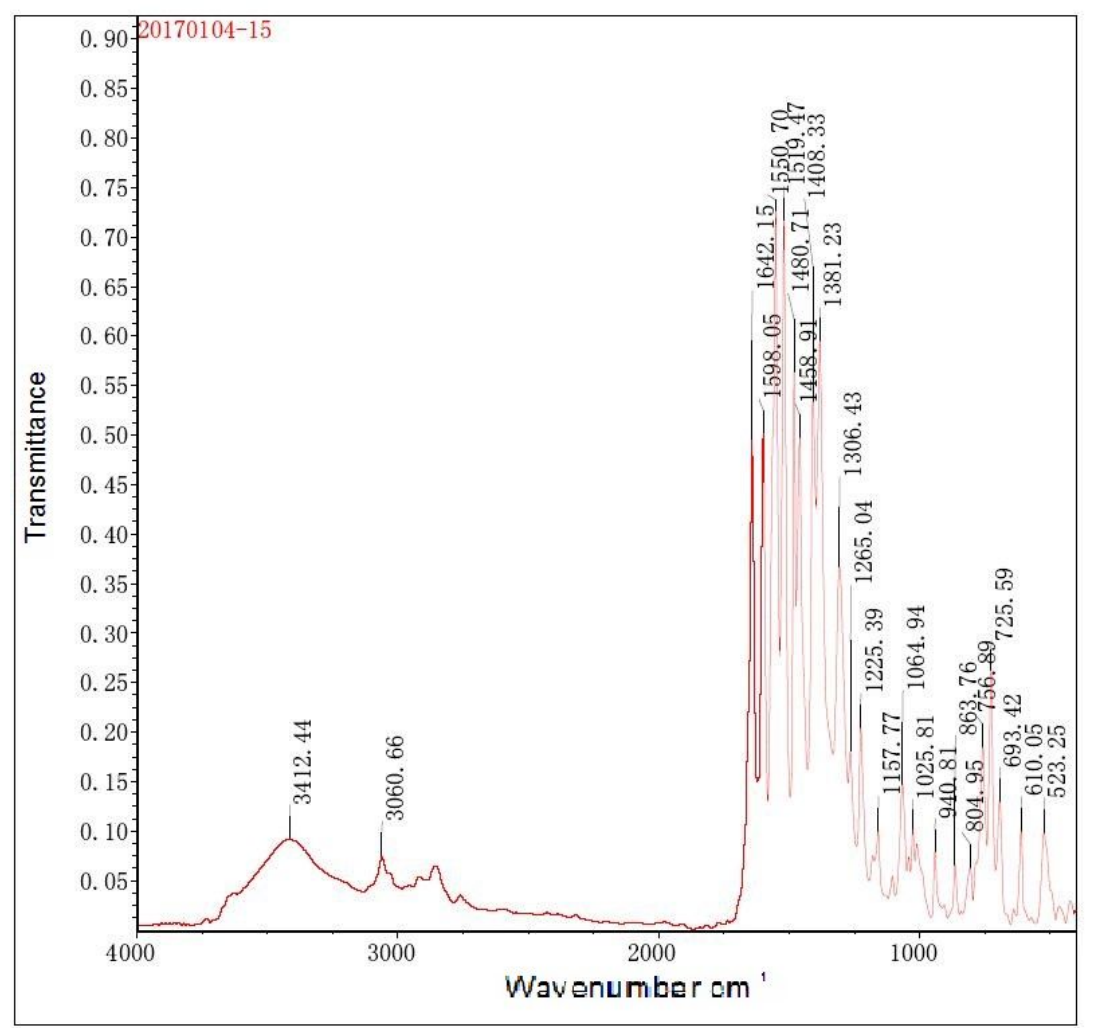

Figure S15. FTIR spectra of 2.

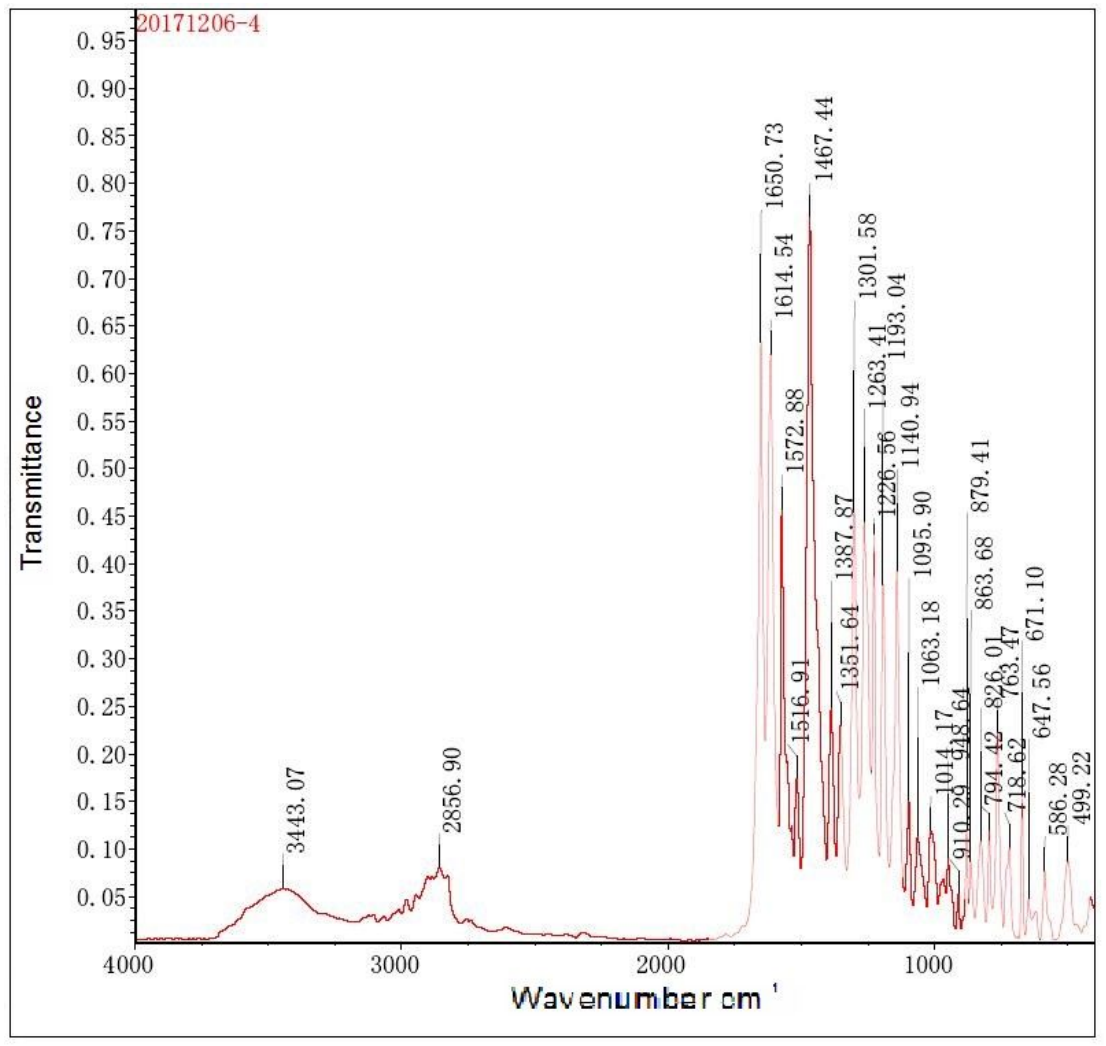

Figure S16. FTIR spectra of 3 . 




Figure S17. FTIR spectra of 4.

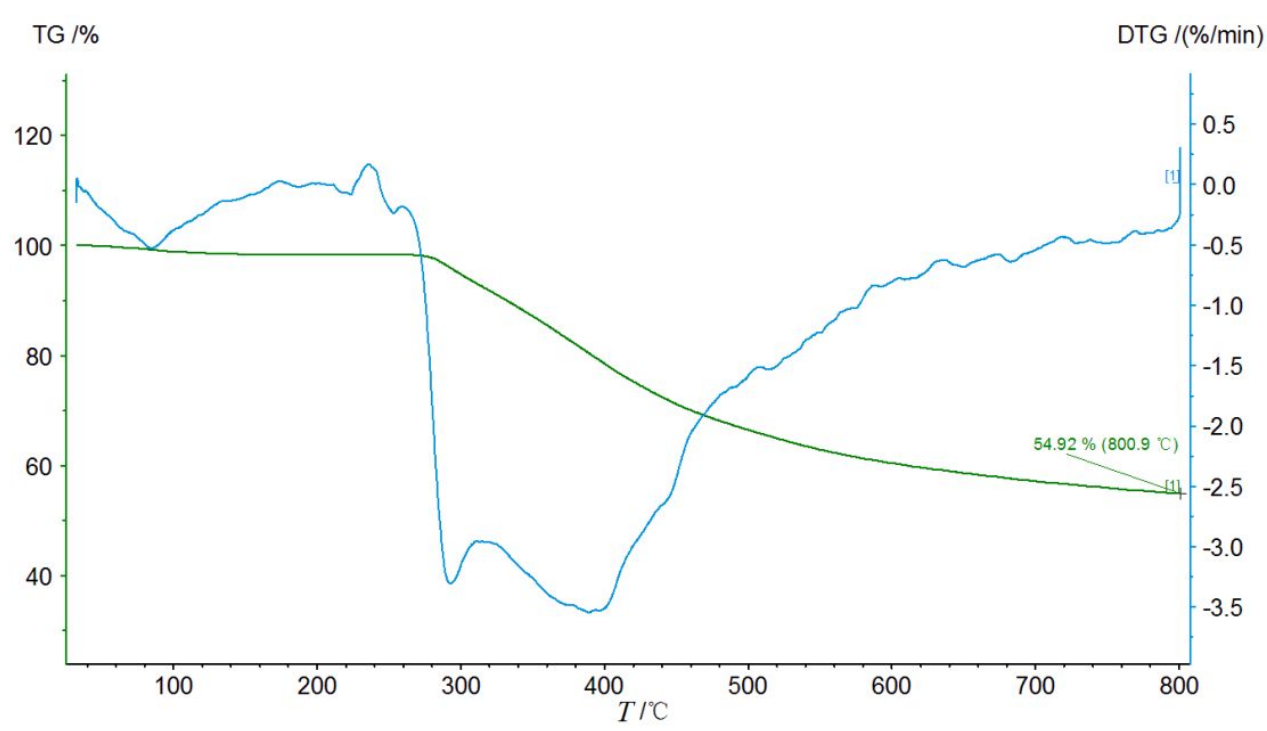

Figure S18. TG/DTG curves of $\mathbf{1}$. 


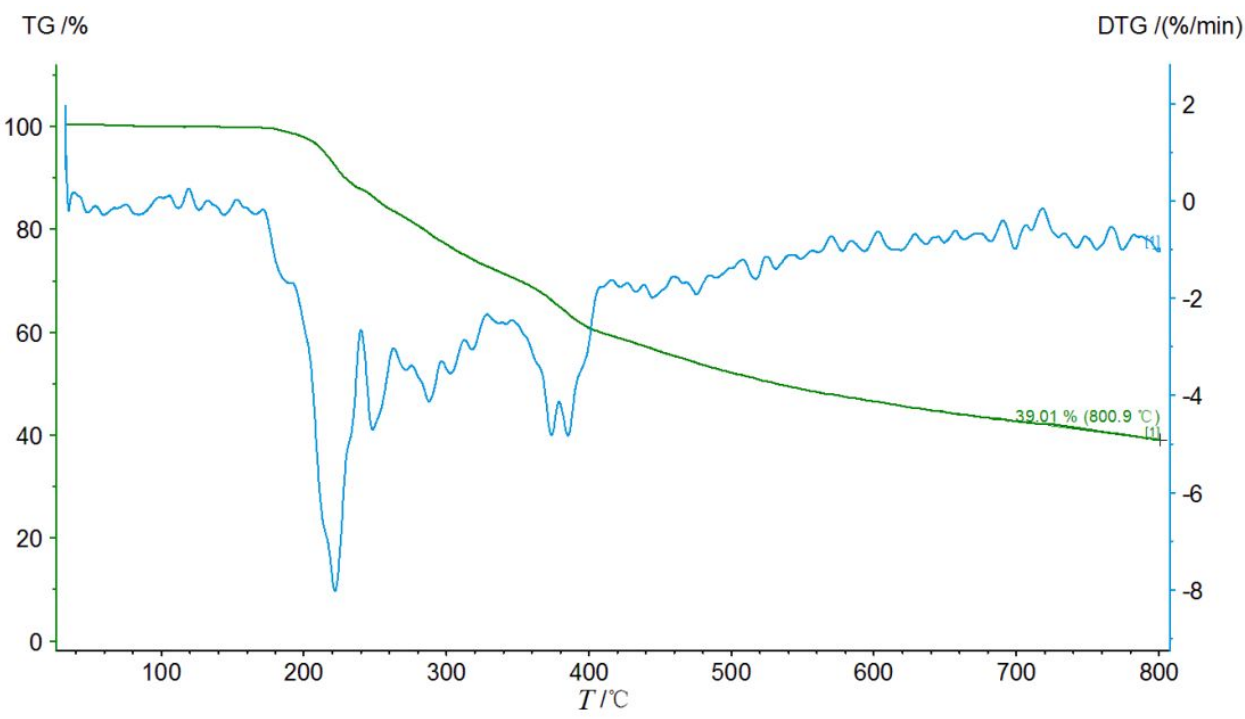

Figure S19. TG/DTG curves of 2.

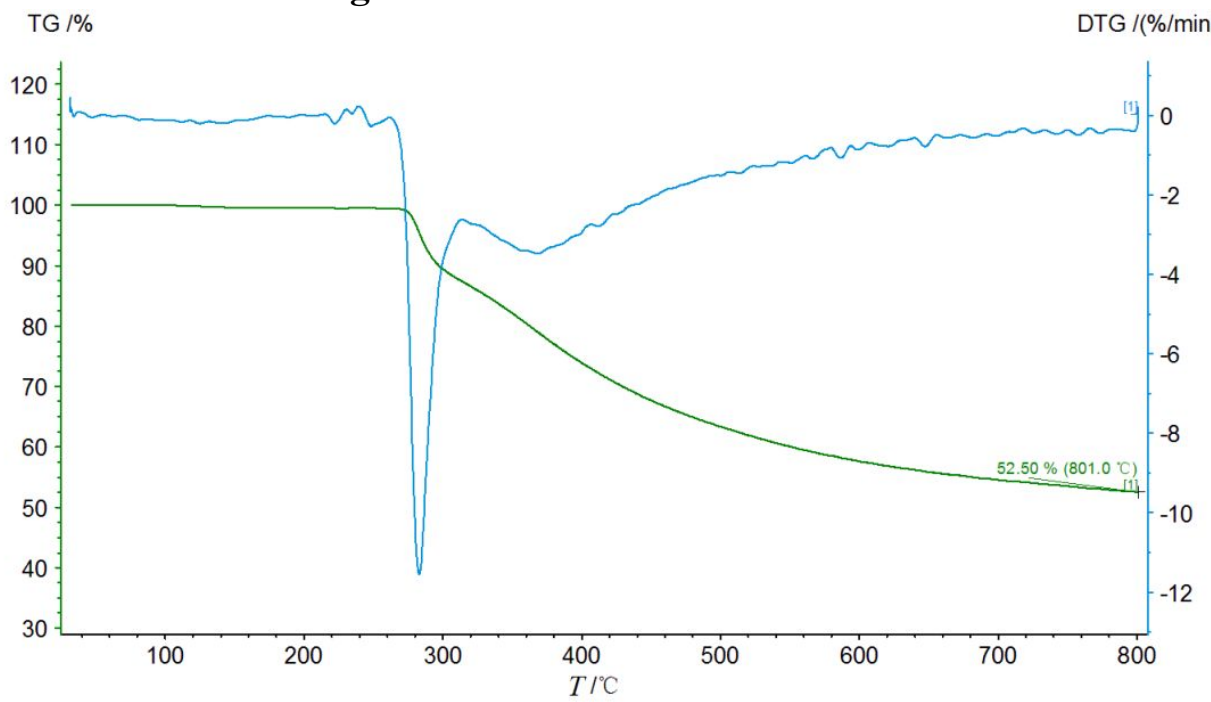

Figure S20. TG/DTG curves of 3.

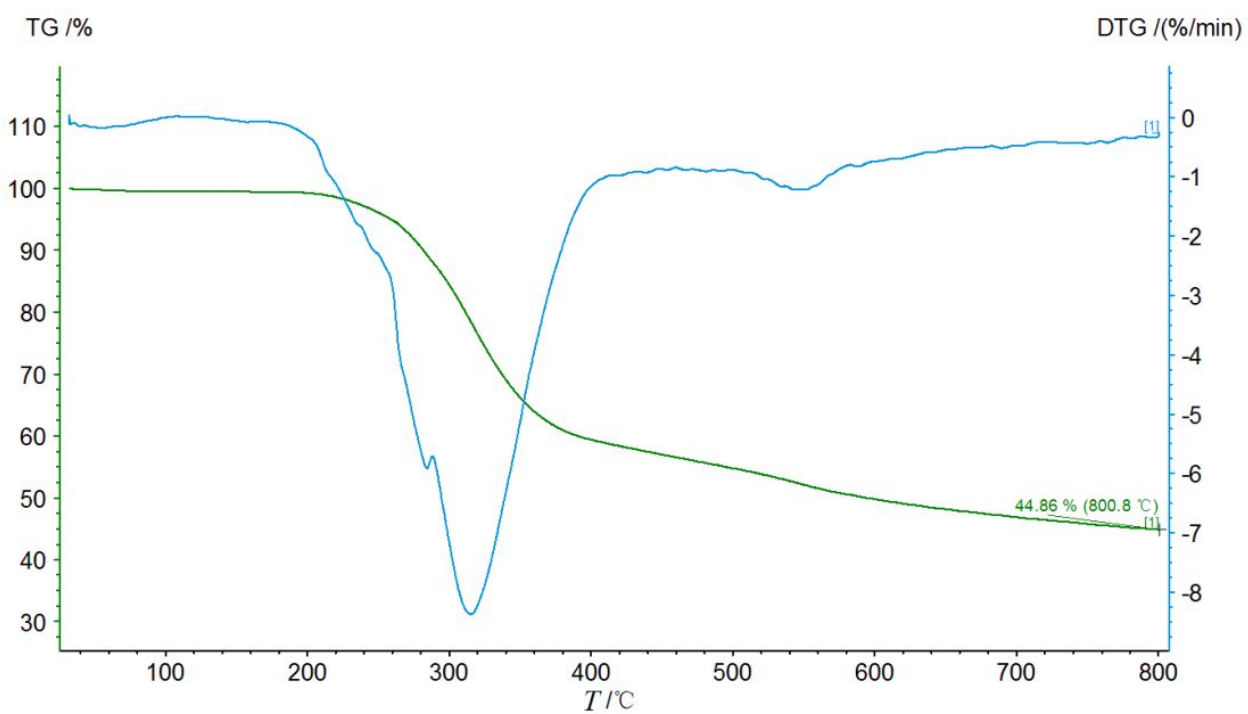


Figure S21. TG/DTG curves of 4.

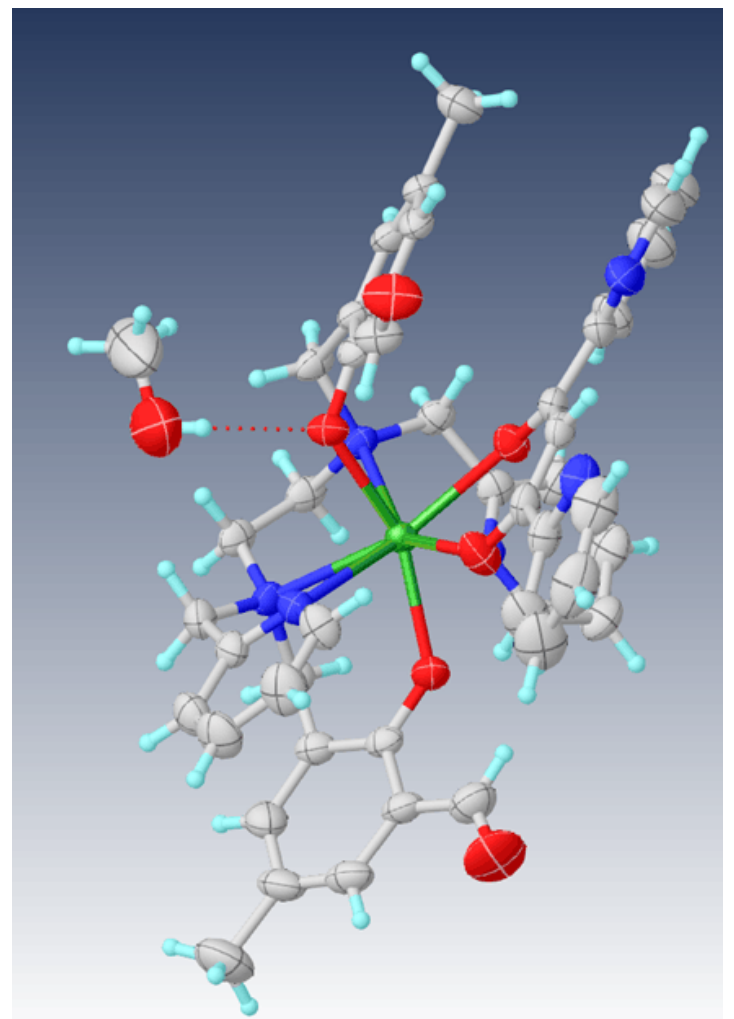

Figure S22. The formation of $\mathrm{O}-\mathrm{H} \cdots \mathrm{O}$ hydrogen bond in compound $\mathbf{1}$.

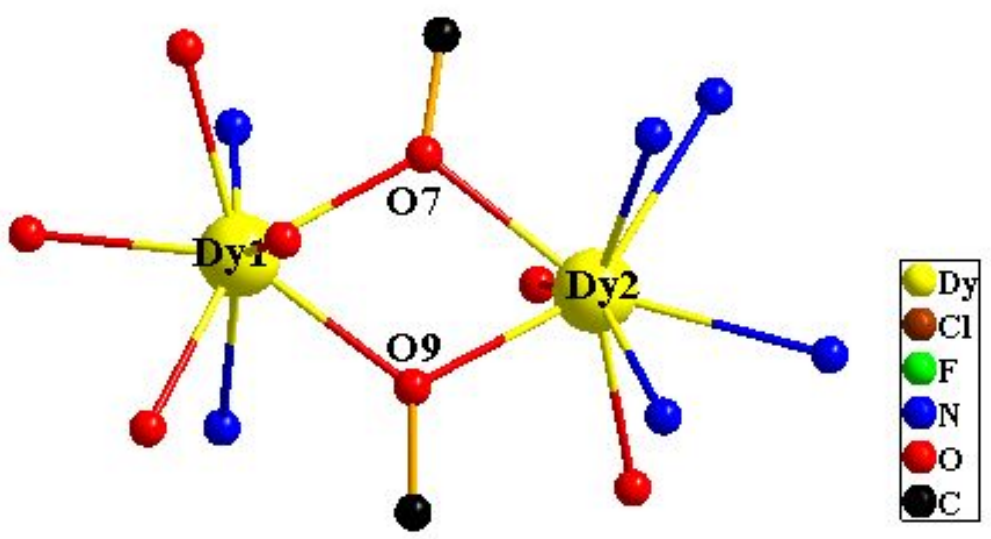

Figure S23. The metal centers in dinuclear core of $\mathbf{4}$ are bridged by two phenol-O atoms (O7 and $\mathrm{O} 9$ ) of one $\mathrm{H}_{2} \mathrm{Lox}^{2-}$ ligand.

(a)

(b)

Figure S24. Local coordination geometries of the Dy (III) ions for 2 (a) and 4 (b).

\section{Magnetic properties for $1-4$}

The zero-field ac susceptibility experiments were determined in the range of 2.0-35.0 $\mathrm{K}$ and at frequencies of 1, 33, 100, 300, 500, 700, 900 and $999 \mathrm{~Hz}$ in 1 (Figure S25). For 2, zero-field ac susceptibilities were measured in the range of 2.0-20.0 K and at frequency of $999 \mathrm{~Hz}$ (Figure S26). The zero-field ac susceptibility experiments were determined in the range of 2.0-25.0 $\mathrm{K}$ and at frequencies of 1,10 , 100, 300, 700 and $999 \mathrm{~Hz}$ in 3 (Figure S27). For 4, zero-field ac susceptibilities were measured in the range of $2.0-20.0 \mathrm{~K}$ and at frequency of $1,100,800$ and $1000 \mathrm{~Hz}$ (Figure S28).

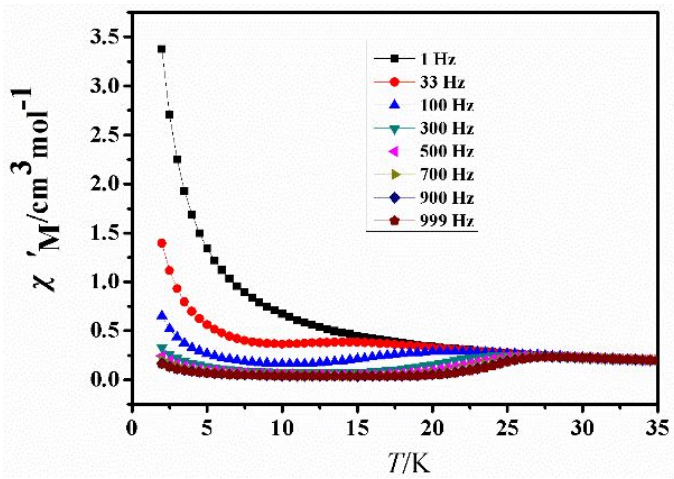

(a)

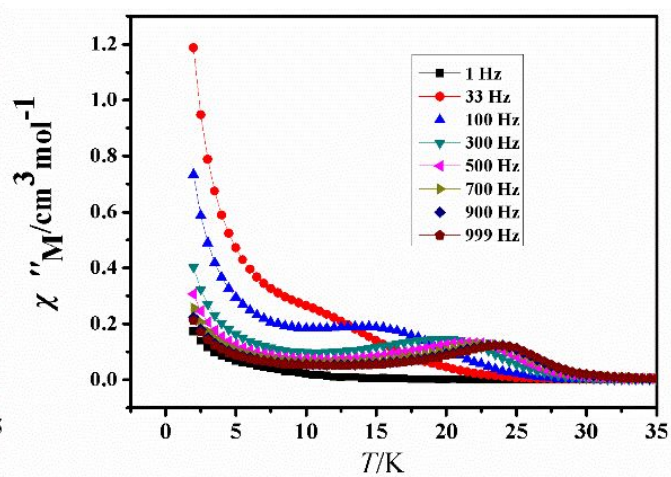

(b)

Figure S25. Temperature dependence of the in-phase $\left(\chi^{\prime}, a\right)$ and out-of-phase $\left(\chi^{\prime \prime}, b\right)$ ac susceptibility signals under 0 Oe dc field for $\mathbf{1}$.

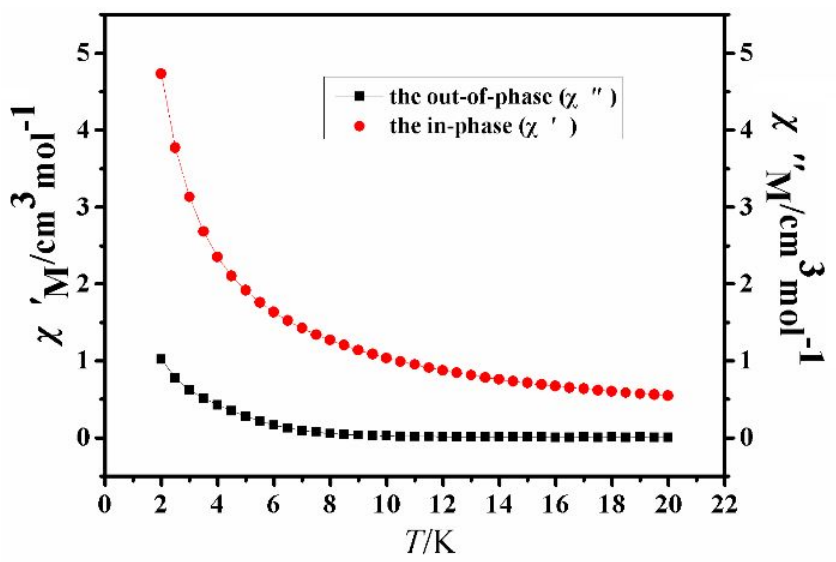

Figure S26. Temperature dependence of the in-phase $\left(\chi^{\prime}, a\right)$ and out-of-phase $\left(\chi^{\prime \prime}, b\right)$ ac susceptibility signals under 0 Oe dc field for 2 . 


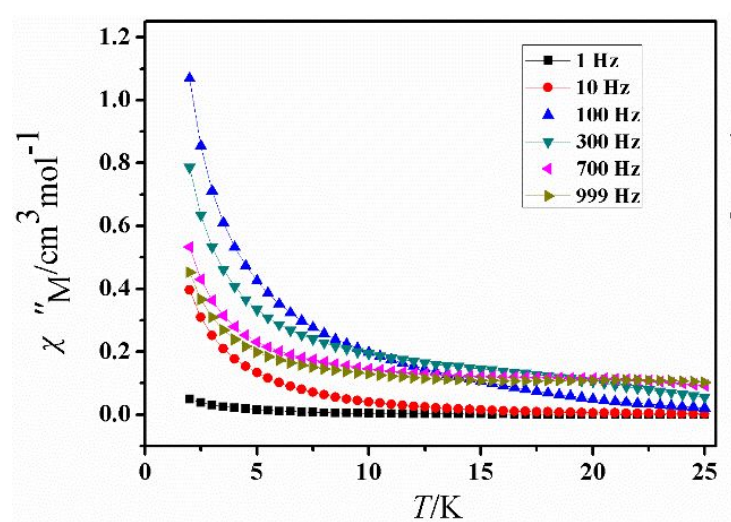

(a)

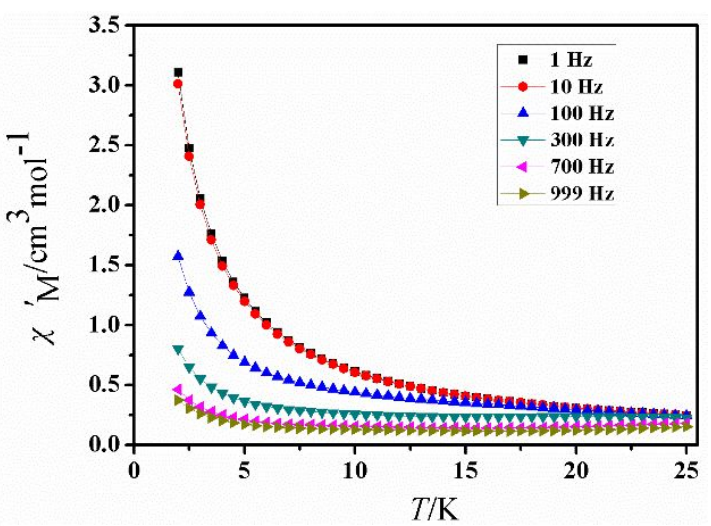

(b)

Figure S27. Temperature dependence of the in-phase $\left(\chi^{\prime}, a\right)$ and out-of-phase $\left(\chi^{\prime \prime}, b\right)$ ac susceptibility signals under 0 Oe dc field for $\mathbf{3}$.

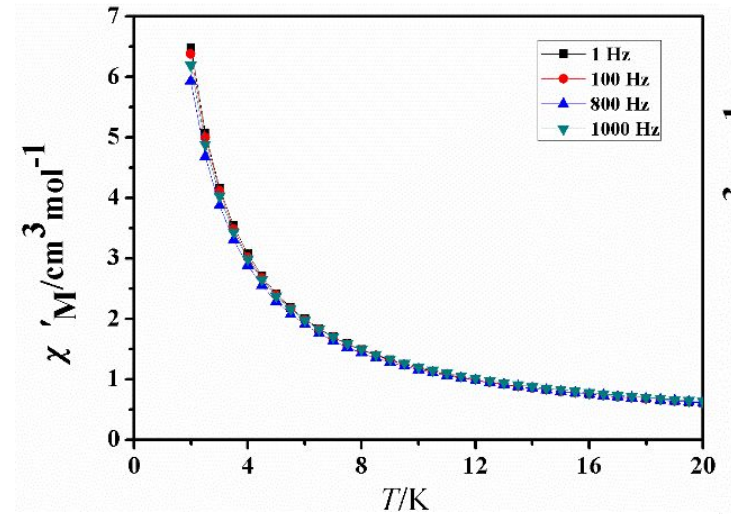

(a)

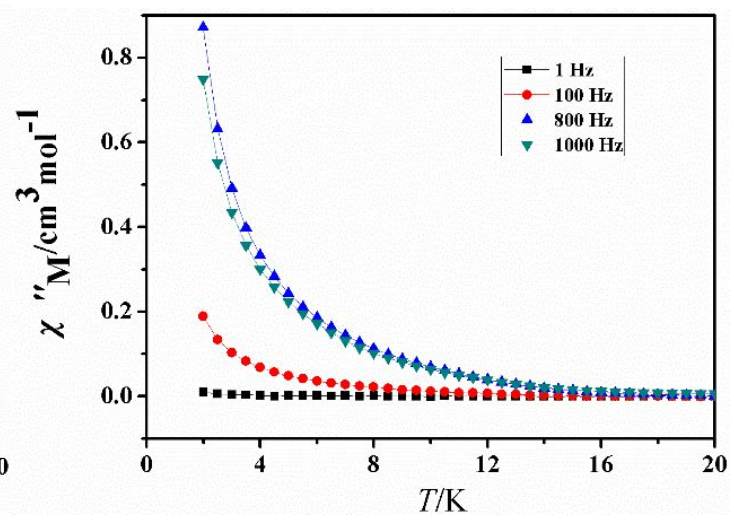

(b)

Figure S28. Temperature dependence of the in-phase $\left(\chi^{\prime}, a\right)$ and out-of-phase $\left(\chi^{\prime \prime}, b\right)$ ac susceptibility signals under 0 Oe dc field for 4 .

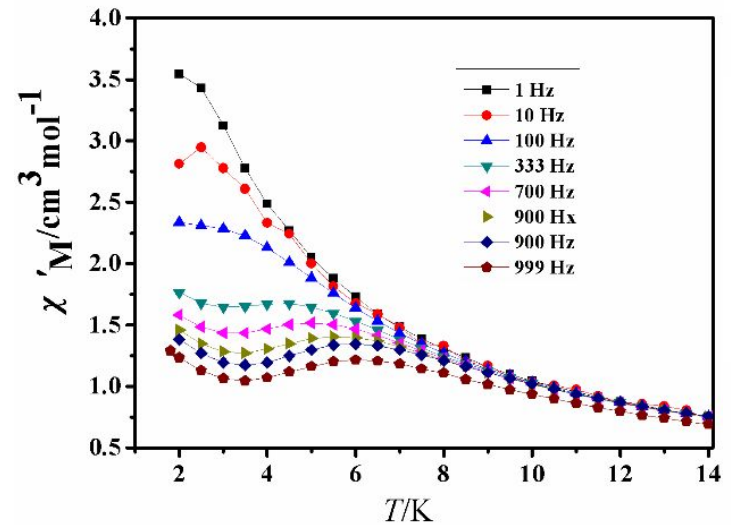

(a)

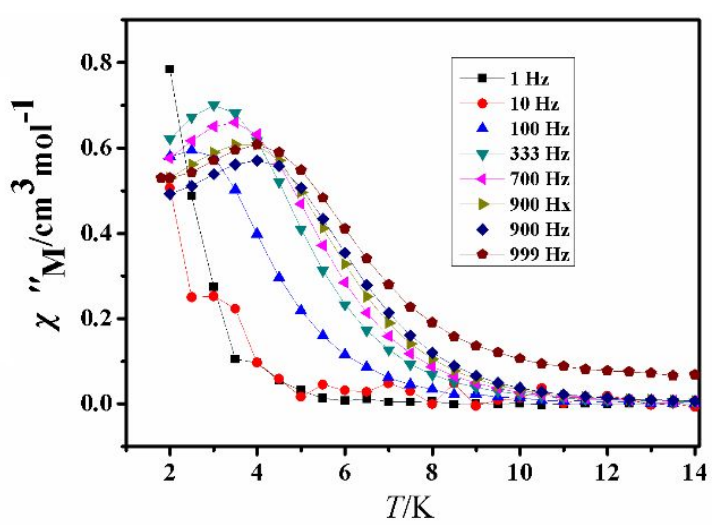

(b)

Figure S29. Temperature dependence of the in-phase $\left(\chi^{\prime}, a\right)$ and out-of-phase $\left(\chi^{\prime \prime}, b\right)$ ac susceptibility signals under 1500 Oe dc field for $\mathbf{2}$. 


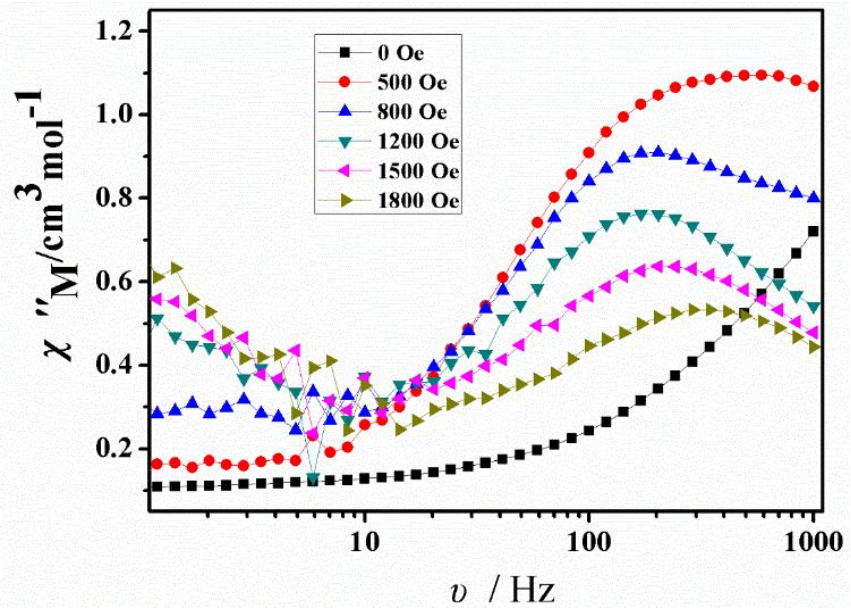

Figure S30. Frequency-dependence of the out-of-phase ac susceptibility under various applied fields at $2.2 \mathrm{~K}$ for $\mathbf{2} . \chi^{\prime \prime}$ signal with significant sharp peaks under 1200 Oe or $1500 \mathrm{dc}$ field indicate field-enhanced slow magnetic relaxation operating in 2 . The solid lines are guides for the eyes.

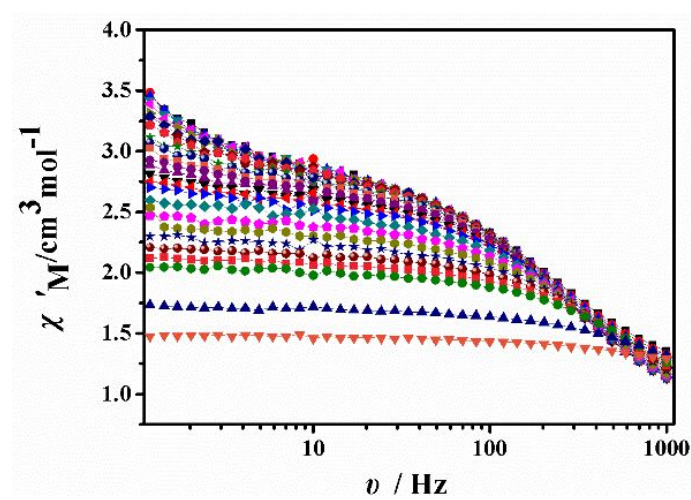

(a)

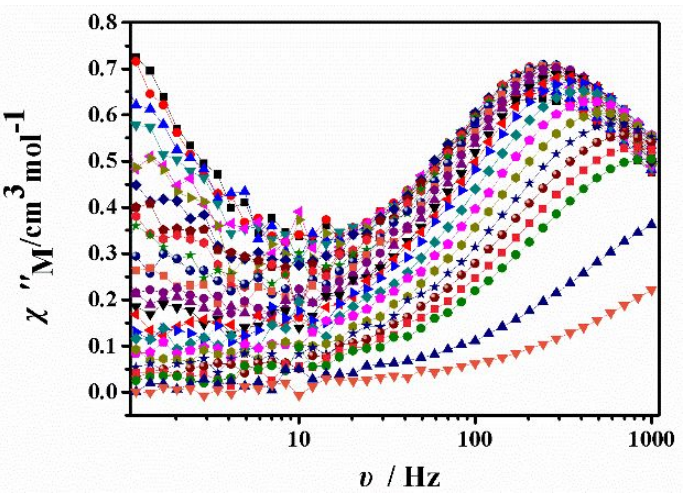

(b)

Figure S31. Plots of the frequency-dependent in-phase $\left(\chi^{\prime}, a\right)$ and out-of-phase $\left(\chi^{\prime \prime}, b\right)$ ac susceptibility from $2.0 \mathrm{~K}$ to $7.0 \mathrm{~K}$ for for 2 under $1500 \mathrm{Oe}$ dc field.

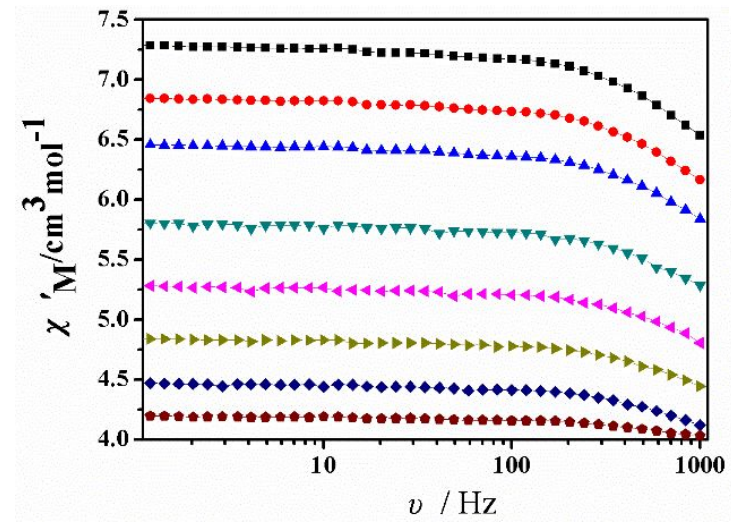

(a)

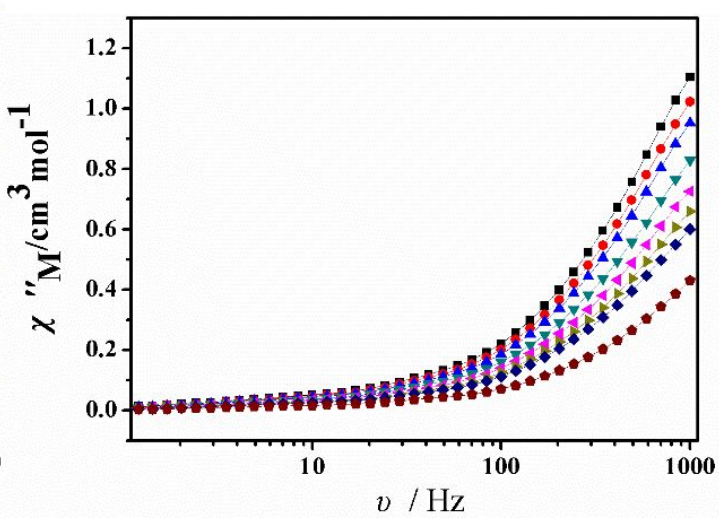

(b)

Figure S32. Plots of the frequency-dependent in-phase $\left(\chi^{\prime}\right.$, a) and out-of-phase $\left(\chi^{\prime \prime}, b\right)$ ac susceptibility from $2.0 \mathrm{~K}$ to $3.0 \mathrm{~K}$ for 4 under 0 Oe dc field. 


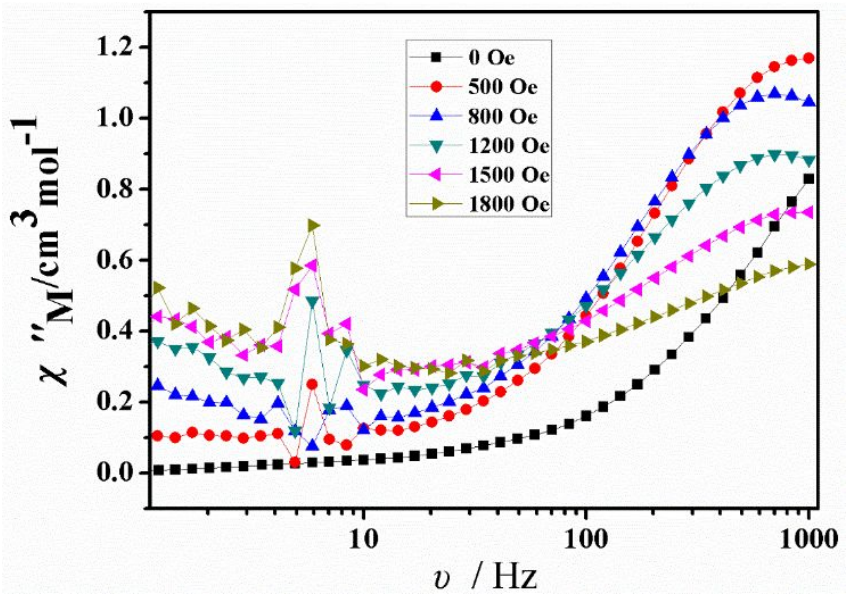

Figure S33. Frequency-dependence of the out-of-phase ac susceptibility under various applied fields at $2.2 \mathrm{~K}$ for 4 . $\chi^{\prime \prime}$ signal with a significant sharp peak under 800 Oe dc field indicate field-enhanced slow magnetic relaxation operating in 4 . The solid lines are guides for the eyes.

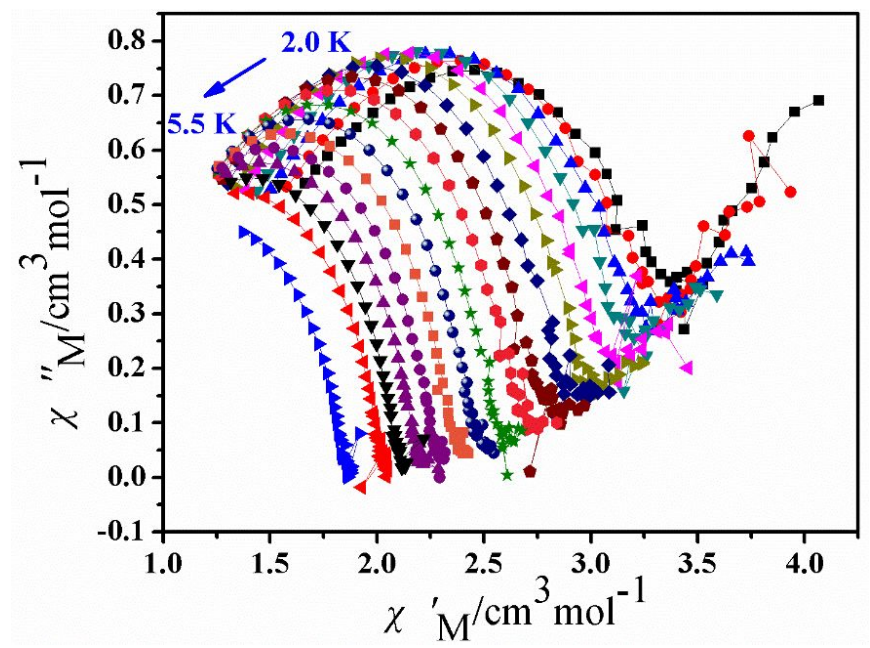

Figure S34. Cole-Cole plots for 2 from $2.0 \mathrm{~K}$ to $5.5 \mathrm{~K}$ using the ac susceptibility data under a 1200 Oe applied dc field.

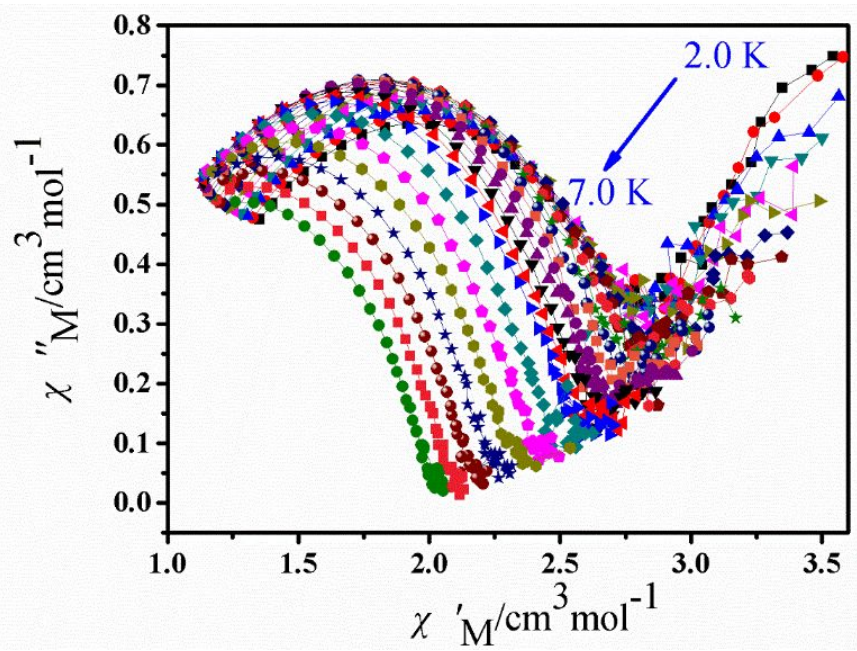

Figure S35. Cole-Cole plots for 2 from $2.0 \mathrm{~K}$ to $7.0 \mathrm{~K}$ using the ac susceptibility data 
under a 1500 Oe applied dc field.

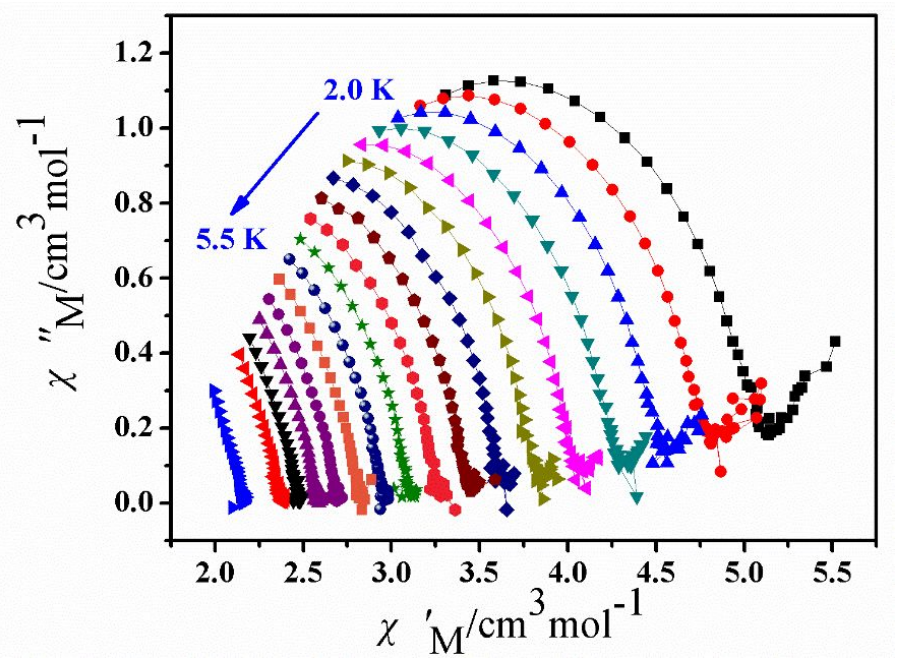

Figure S36. Cole-Cole plots for 4 from $2.0 \mathrm{~K}$ to $5.5 \mathrm{~K}$ using the ac susceptibility data under a 800 Oe applied dc field.

\section{Relaxation fitting parameters of 1 and 3}

The magnetic susceptibility data of $\mathbf{1}$ and $\mathbf{3}$ under a zero dc field were described by the modified Debye functions:

$$
\begin{aligned}
& \chi^{\prime}(\omega)=\chi_{\mathrm{S}}+\left(\chi_{\mathrm{T}}-\chi_{\mathrm{S}}\right) \frac{1+(\omega \tau)^{1-\alpha} \sin \left(\frac{\pi}{2} \alpha\right)}{1+2(\omega \tau)^{1-\alpha} \sin \left(\frac{\pi}{2} \alpha\right)+(\omega \tau)^{(2-2 \alpha)}} \\
& \chi^{\prime \prime}(\omega)=\left(\chi_{\mathrm{T}}-\chi_{\mathrm{S}}\right) \frac{(\omega \tau)^{1-\alpha} \cos \left(\frac{\pi}{2} \alpha\right)}{1+2(\omega \tau)^{1-\alpha} \sin \left(\frac{\pi}{2} \alpha\right)+(\omega \tau)^{(2-2 \alpha)}} \\
& \chi_{\omega=\tau^{-1}}^{\prime \prime}=\left(\chi_{\mathrm{T}}-\chi_{\mathrm{S}}\right) \frac{\cos \left(\frac{\pi}{2} \alpha\right)}{2+2 \sin \left(\frac{\pi}{2} \alpha\right)}=\frac{1}{2}\left(\chi_{\mathrm{T}}-\chi_{\mathrm{S}}\right) \tan \frac{\pi}{4}(1-\alpha)
\end{aligned}
$$




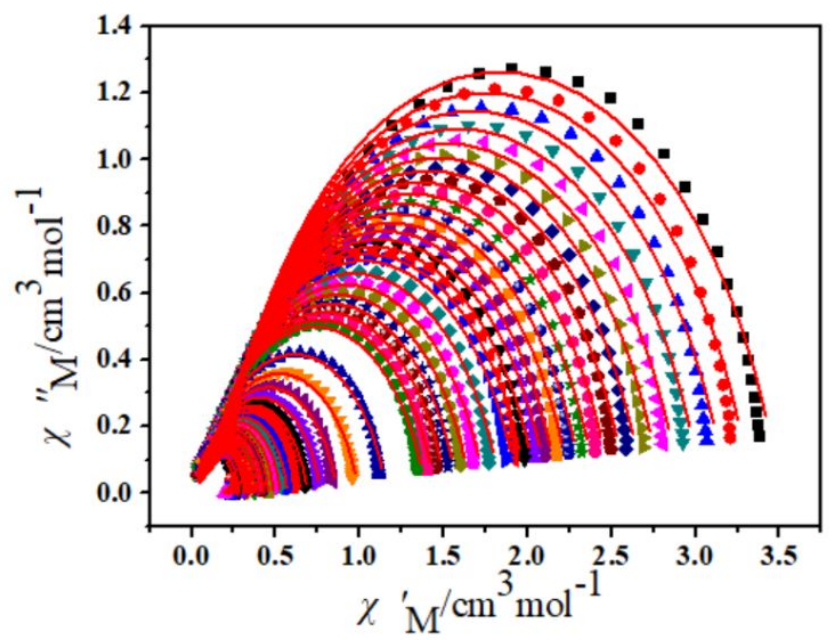

Figure S37. The red solid lines are the best fits for the generalized Debye model between $2.0 \mathrm{~K}$ and $11.0 \mathrm{~K}$ in 1.

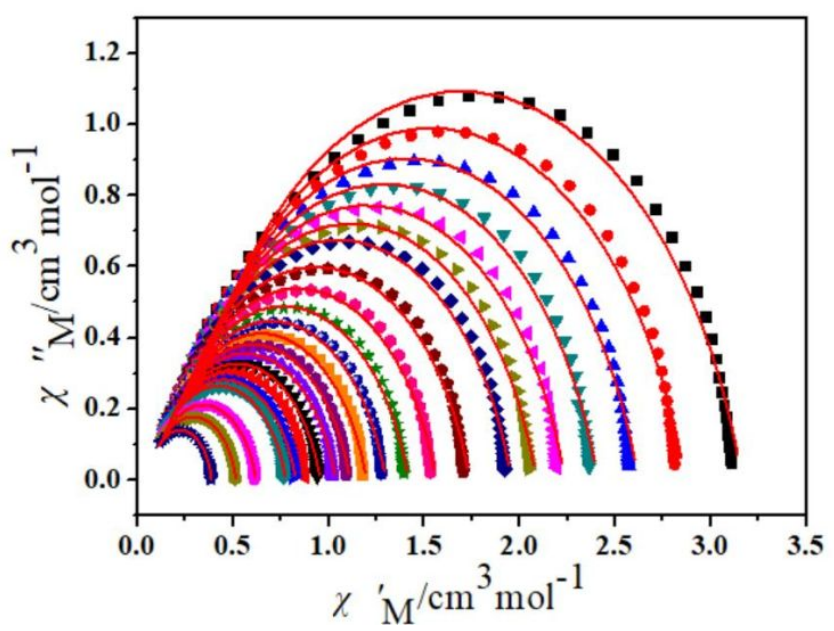

Figure S38. The red solid lines are the best fits for the generalized Debye model between $2.0 \mathrm{~K}$ and $16.0 \mathrm{~K}$ in 3 .

In principle, this parameter $\tau$ has contributions from different mechanisms, e.g, Orbach, Raman as well as QTM.

For the magnetic relaxation of Kramers system under zero applied field, the direct process can be neglected. As shown in Fig. S15 and S16, the coexistence of several mechanisms is verified since a reasonable fit, covering the whole temperature range in 1 or 3. It is obtained by combining Orbach, Raman and QTM paths (eqn (S1)):

$$
\tau^{-1}=\tau_{Q T M}^{-1}+C T^{n}+\tau_{0}^{-1} \exp \left(-U_{\text {eff }} / k T\right) \text { eqn (S1) }
$$

The three terms correspond to the QTM process, the Raman process and the Orbach process where $\tau$ is the inverse of the ac frequency, $T$ is the temperature of the maximum in the ac signal, $U_{\text {eff }}$ is the effective energy barrier, $k$ is Boltzmann's 
constant (Table S5). The fit in the temperature range $T=2.0-35.0 \mathrm{~K}$ for compound 1 by eqn (1) resulted in $\tau_{\mathrm{QTM}}=0.0072 \mathrm{~s}, n=3.89, C=0.01 \mathrm{~s}^{-1} \mathrm{~K}^{-3.89}, \tau_{0}=1.16 \times 10^{-8} \mathrm{~s}$ and an effective energy barrier of $U_{\text {eff }}=247.3 \mathrm{~K}$. The fit in the temperature range $\mathrm{T}=$ $2.0-16.0 \mathrm{~K}$ for compound 3 by eqn (1) resulted in $\tau_{\mathrm{QTM}}=0.0018 \mathrm{~s}, n=1.94, C=6.41$ $\mathrm{s}^{-1} \mathrm{~K}^{-1.94}, \tau_{0}=6.74 \times 10^{-6} \mathrm{~s}$ and an effective energy barrier of $U_{\text {eff }}=78.0 \mathrm{~K}$.

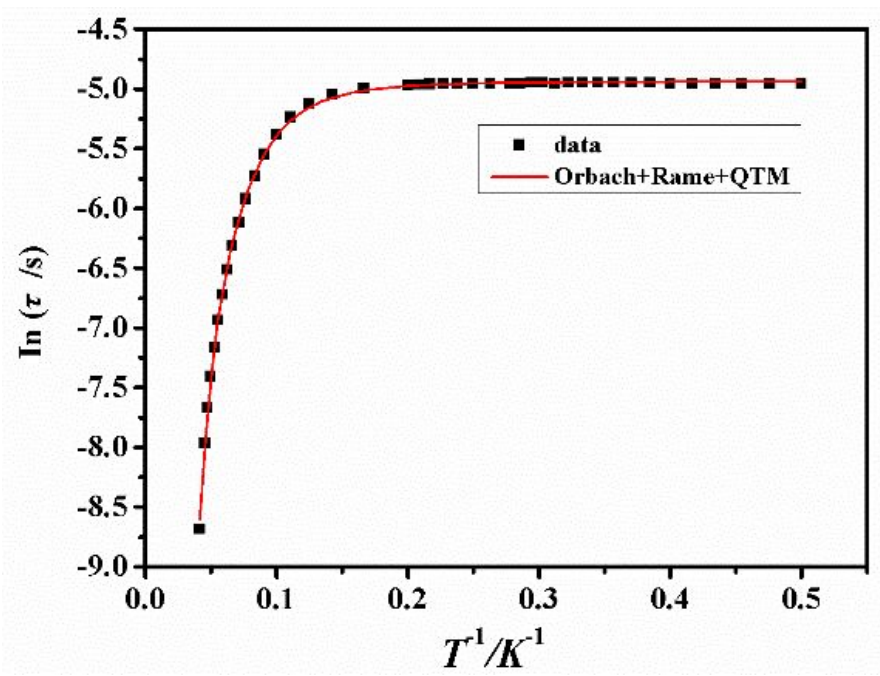

Figure S39. Fitting of frequency dependence of relaxation times under a zero dc field for 1 .

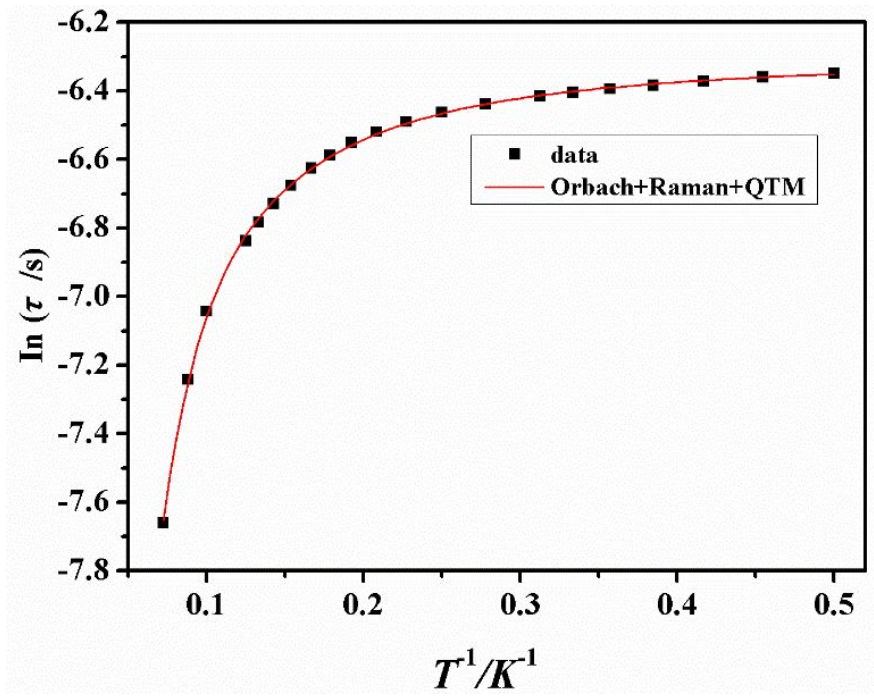

Figure S40. Fitting of frequency dependence of relaxation times under a zero dc field for 3 .

Table S3 Relaxation fitting parameters from Least-Squares Fitting of $\chi(\omega)$ data for $\mathbf{1}$ under a zero applied dc field.

\begin{tabular}{|c|c|c|c|c|}
\hline$T$ & $\Delta \chi_{1}\left(\mathrm{~cm}^{3} \mathrm{~mol}^{-1}\right)$ & $\Delta \chi_{2}\left(\mathrm{~cm}^{3} \mathrm{~mol}^{-1}\right)$ & $\tau(\mathrm{s})$ & $\alpha$ \\
\hline 2 & $0.172184 \mathrm{E}+00$ & $0.350095 \mathrm{E}+01$ & $0.705074 \mathrm{E}-02$ & $0.173094 \mathrm{E}+00$ \\
\hline 2.1 & $0.163860 \mathrm{E}+00$ & $0.333038 \mathrm{E}+01$ & $0.705800 \mathrm{E}-02$ & $0.173290 \mathrm{E}+00$ \\
\hline
\end{tabular}




\begin{tabular}{|c|c|c|c|c|}
\hline 2.2 & $0.156535 \mathrm{E}+00$ & $0.318050 \mathrm{E}+01$ & $0.706683 \mathrm{E}-02$ & $0.173654 \mathrm{E}+00$ \\
\hline 2.3 & $0.149411 \mathrm{E}+00$ & $0.303582 \mathrm{E}+01$ & $0.707163 \mathrm{E}-02$ & $0.173967 \mathrm{E}+00$ \\
\hline 2.4 & $0.143350 \mathrm{E}+00$ & $0.290872 \mathrm{E}+01$ & $0.707623 \mathrm{E}-02$ & $0.174070 \mathrm{E}+00$ \\
\hline 2.5 & $0.137873 \mathrm{E}+00$ & $0.279425 \mathrm{E}+01$ & $0.708608 \mathrm{E}-02$ & $0.174324 \mathrm{E}+00$ \\
\hline 2.6 & $0.132571 \mathrm{E}+00$ & $0.268841 \mathrm{E}+01$ & $0.709226 \mathrm{E}-02$ & $0.174792 \mathrm{E}+00$ \\
\hline 2.7 & $0.128161 \mathrm{E}+00$ & $0.259023 \mathrm{E}+01$ & $0.709806 \mathrm{E}-02$ & $0.174777 \mathrm{E}+00$ \\
\hline 2.8 & $0.123862 \mathrm{E}+00$ & $0.249703 \mathrm{E}+01$ & $0.709450 \mathrm{E}-02$ & $0.174833 \mathrm{E}+00$ \\
\hline 2.9 & $0.119525 \mathrm{E}+00$ & $0.241193 \mathrm{E}+01$ & $0.709507 \mathrm{E}-02$ & $0.175027 \mathrm{E}+00$ \\
\hline 3 & $0.115253 \mathrm{E}+00$ & $0.233173 \mathrm{E}+01$ & $0.709781 \mathrm{E}-02$ & $0.175584 \mathrm{E}+00$ \\
\hline 3.1 & $0.112228 \mathrm{E}+00$ & $0.225794 \mathrm{E}+01$ & $0.710282 \mathrm{E}-02$ & $0.175509 \mathrm{E}+00$ \\
\hline 3.2 & $0.108798 \mathrm{E}+00$ & $0.218559 \mathrm{E}+01$ & $0.709099 \mathrm{E}-02$ & $0.175263 \mathrm{E}+00$ \\
\hline 3.3 & $0.105663 \mathrm{E}+00$ & $0.212019 \mathrm{E}+01$ & $0.709350 \mathrm{E}-02$ & $0.175343 \mathrm{E}+00$ \\
\hline 3.4 & $0.102565 \mathrm{E}+00$ & $0.205728 \mathrm{E}+01$ & $0.709838 \mathrm{E}-02$ & $0.175742 \mathrm{E}+00$ \\
\hline 3.5 & $0.995502 \mathrm{E}-01$ & $0.199788 \mathrm{E}+01$ & $0.708982 \mathrm{E}-02$ & $0.176055 \mathrm{E}+00$ \\
\hline 3.6 & $0.967505 \mathrm{E}-01$ & $0.194280 \mathrm{E}+01$ & $0.709042 \mathrm{E}-02$ & $0.176560 \mathrm{E}+00$ \\
\hline 3.8 & $0.918231 \mathrm{E}-01$ & $0.183871 \mathrm{E}+01$ & $0.707493 \mathrm{E}-02$ & $0.176188 \mathrm{E}+00$ \\
\hline 4 & $0.876733 \mathrm{E}-01$ & $0.174546 \mathrm{E}+01$ & $0.706632 \mathrm{E}-02$ & $0.176316 \mathrm{E}+00$ \\
\hline 4.2 & $0.835681 \mathrm{E}-01$ & $0.166218 \mathrm{E}+01$ & $0.705614 \mathrm{E}-02$ & $0.176453 \mathrm{E}+00$ \\
\hline 4.4 & $0.799746 \mathrm{E}-01$ & $0.158583 \mathrm{E}+01$ & $0.704006 \mathrm{E}-02$ & $0.176661 \mathrm{E}+00$ \\
\hline 4.6 & $0.767012 \mathrm{E}-01$ & $0.151594 \mathrm{E}+01$ & $0.701805 \mathrm{E}-02$ & $0.176598 \mathrm{E}+00$ \\
\hline 4.8 & $0.735991 \mathrm{E}-01$ & $0.145171 \mathrm{E}+01$ & $0.698682 \mathrm{E}-02$ & $0.176482 \mathrm{E}+00$ \\
\hline 5 & $0.707610 \mathrm{E}-01$ & $0.139350 \mathrm{E}+01$ & $0.696323 \mathrm{E}-02$ & $0.176509 \mathrm{E}+00$ \\
\hline 6 & $0.599132 \mathrm{E}-01$ & $0.115895 \mathrm{E}+01$ & $0.677193 \mathrm{E}-02$ & $0.174829 \mathrm{E}+00$ \\
\hline 7 & $0.523943 \mathrm{E}-01$ & $0.991799 \mathrm{E}+00$ & $0.644446 \mathrm{E}-02$ & $0.170104 \mathrm{E}+00$ \\
\hline 8 & $0.471577 \mathrm{E}-01$ & $0.865602 \mathrm{E}+00$ & $0.595332 \mathrm{E}-02$ & $0.160468 \mathrm{E}+00$ \\
\hline 9 & $0.431029 \mathrm{E}-01$ & $0.766452 \mathrm{E}+00$ & $0.531917 \mathrm{E}-02$ & $0.146923 \mathrm{E}+00$ \\
\hline 10 & $0.392427 \mathrm{E}-01$ & $0.687468 \mathrm{E}+00$ & $0.460369 \mathrm{E}-02$ & $0.132133 \mathrm{E}+00$ \\
\hline 11 & $0.361927 \mathrm{E}-01$ & $0.622086 \mathrm{E}+00$ & $0.390182 \mathrm{E}-02$ & $0.116236 \mathrm{E}+00$ \\
\hline 12 & $0.326010 \mathrm{E}-01$ & $0.568985 \mathrm{E}+00$ & $0.325381 \mathrm{E}-02$ & $0.104332 \mathrm{E}+00$ \\
\hline 13 & $0.294241 \mathrm{E}-01$ & $0.523604 \mathrm{E}+00$ & $0.269225 \mathrm{E}-02$ & $0.933358 \mathrm{E}-01$ \\
\hline 14 & $0.264422 \mathrm{E}-01$ & $0.485572 \mathrm{E}+00$ & $0.221019 \mathrm{E}-02$ & $0.851029 \mathrm{E}-01$ \\
\hline 15 & $0.236456 \mathrm{E}-01$ & $0.452837 \mathrm{E}+00$ & $0.180944 \mathrm{E}-02$ & $0.783216 \mathrm{E}-01$ \\
\hline 16 & $0.210792 \mathrm{E}-01$ & $0.424711 \mathrm{E}+00$ & $0.148402 \mathrm{E}-02$ & $0.736479 \mathrm{E}-01$ \\
\hline 17 & $0.187488 \mathrm{E}-01$ & $0.399513 \mathrm{E}+00$ & $0.120748 \mathrm{E}-02$ & $0.693161 \mathrm{E}-01$ \\
\hline 18 & $0.166086 \mathrm{E}-01$ & $0.377164 \mathrm{E}+00$ & $0.974348 \mathrm{E}-03$ & $0.662979 \mathrm{E}-01$ \\
\hline 19 & $0.147509 \mathrm{E}-01$ & $0.357230 \mathrm{E}+00$ & $0.775814 \mathrm{E}-03$ & $0.650069 \mathrm{E}-01$ \\
\hline 20 & $0.133889 \mathrm{E}-01$ & $0.339361 \mathrm{E}+00$ & $0.608271 \mathrm{E}-03$ & $0.640469 \mathrm{E}-01$ \\
\hline 21 & $0.124252 \mathrm{E}-01$ & $0.323414 \mathrm{E}+00$ & $0.465935 \mathrm{E}-03$ & $0.652618 \mathrm{E}-01$ \\
\hline 22 & $0.124118 \mathrm{E}-01$ & $0.308606 \mathrm{E}+00$ & $0.346672 \mathrm{E}-03$ & $0.641322 \mathrm{E}-01$ \\
\hline 24 & $0.101195 \mathrm{E}-01$ & $0.282846 \mathrm{E}+00$ & $0.169361 \mathrm{E}-03$ & $0.653072 \mathrm{E}-01$ \\
\hline 26 & $0.347720 \mathrm{E}-10$ & $0.261410 \mathrm{E}+00$ & $0.692081 \mathrm{E}-04$ & $0.682697 \mathrm{E}-01$ \\
\hline 28 & $0.632461 \mathrm{E}-10$ & $0.243326 \mathrm{E}+00$ & $0.282057 \mathrm{E}-04$ & $0.651858 \mathrm{E}-01$ \\
\hline 30 & $0.153967 \mathrm{E}-09$ & $0.227241 \mathrm{E}+00$ & $0.133446 \mathrm{E}-04$ & $0.682356 \mathrm{E}-01$ \\
\hline
\end{tabular}




\begin{tabular}{|l|l|l|l|l|}
\hline 32 & $0.242323 \mathrm{E}-09$ & $0.213402 \mathrm{E}+00$ & $0.799957 \mathrm{E}-05$ & $0.651179 \mathrm{E}-01$ \\
\hline 35 & $0.338229 \mathrm{E}-09$ & $0.195728 \mathrm{E}+00$ & $0.442700 \mathrm{E}-05$ & $0.674854 \mathrm{E}-01$ \\
\hline
\end{tabular}

Table S4 Relaxation fitting parameters from Least-Squares Fitting of $\chi(\omega)$ data for 3 under a zero applied dc field.

\begin{tabular}{|c|c|c|c|c|}
\hline$T$ & $\Delta \chi_{1}\left(\mathrm{~cm}^{3} \mathrm{~mol}^{-1}\right)$ & $\Delta \chi_{2}\left(\mathrm{~cm}^{3} \mathrm{~mol}^{-1}\right)$ & $\tau(\mathrm{s})$ & $\alpha$ \\
\hline 2 & $0.224362 \mathrm{E}+00$ & $0.316407 \mathrm{E}+01$ & $0.174979 \mathrm{E}-02$ & $0.185730 \mathrm{E}+00$ \\
\hline 2.2 & $0.204196 \mathrm{E}+00$ & $0.286532 \mathrm{E}+01$ & $0.172923 \mathrm{E}-02$ & $0.185622 \mathrm{E}+00$ \\
\hline 2.4 & $0.187216 \mathrm{E}+00$ & $0.262072 \mathrm{E}+01$ & $0.170951 \mathrm{E}-02$ & $0.185503 \mathrm{E}+00$ \\
\hline 2.6 & $0.174083 \mathrm{E}+00$ & $0.240812 \mathrm{E}+01$ & $0.168997 \mathrm{E}-02$ & $0.184435 \mathrm{E}+00$ \\
\hline 2.8 & $0.162149 \mathrm{E}+00$ & $0.223632 \mathrm{E}+01$ & $0.167223 \mathrm{E}-02$ & $0.184711 \mathrm{E}+00$ \\
\hline 3 & $0.152760 \mathrm{E}+00$ & $0.208750 \mathrm{E}+01$ & $0.165495 \mathrm{E}-02$ & $0.184131 \mathrm{E}+00$ \\
\hline 3.2 & $0.143705 \mathrm{E}+00$ & $0.195639 \mathrm{E}+01$ & $0.163746 \mathrm{E}-02$ & $0.184392 \mathrm{E}+00$ \\
\hline 3.6 & $0.129244 \mathrm{E}+00$ & $0.173648 \mathrm{E}+01$ & $0.159974 \mathrm{E}-02$ & $0.184117 \mathrm{E}+00$ \\
\hline 4 & $0.118015 \mathrm{E}+00$ & $0.156078 \mathrm{E}+01$ & $0.156085 \mathrm{E}-02$ & $0.183862 \mathrm{E}+00$ \\
\hline 4.4 & $0.109083 \mathrm{E}+00$ & $0.141771 \mathrm{E}+01$ & $0.151925 \mathrm{E}-02$ & $0.183341 \mathrm{E}+00$ \\
\hline 4.8 & $0.101731 \mathrm{E}+00$ & $0.129850 \mathrm{E}+01$ & $0.147544 \mathrm{E}-02$ & $0.182967 \mathrm{E}+00$ \\
\hline 5.2 & $0.954265 \mathrm{E}-01$ & $0.119754 \mathrm{E}+01$ & $0.142747 \mathrm{E}-02$ & $0.182582 \mathrm{E}+00$ \\
\hline 5.6 & $0.899808 \mathrm{E}-01$ & $0.111125 \mathrm{E}+01$ & $0.137797 \mathrm{E}-02$ & $0.182670 \mathrm{E}+00$ \\
\hline 6 & $0.857028 \mathrm{E}-01$ & $0.103672 \mathrm{E}+01$ & $0.132716 \mathrm{E}-02$ & $0.182107 \mathrm{E}+00$ \\
\hline 6.5 & $0.811151 \mathrm{E}-01$ & $0.956056 \mathrm{E}+00$ & $0.126120 \mathrm{E}-02$ & $0.181647 \mathrm{E}+00$ \\
\hline 7 & $0.776678 \mathrm{E}-01$ & $0.887131 \mathrm{E}+00$ & $0.119617 \mathrm{E}-02$ & $0.180904 \mathrm{E}+00$ \\
\hline 7.5 & $0.749548 \mathrm{E}-01$ & $0.827728 \mathrm{E}+00$ & $0.113320 \mathrm{E}-02$ & $0.180080 \mathrm{E}+00$ \\
\hline 8 & $0.730688 \mathrm{E}-01$ & $0.775625 \mathrm{E}+00$ & $0.107407 \mathrm{E}-02$ & $0.178728 \mathrm{E}+00$ \\
\hline 10 & $0.700012 \mathrm{E}-01$ & $0.619154 \mathrm{E}+00$ & $0.873834 \mathrm{E}-03$ & $0.165435 \mathrm{E}+00$ \\
\hline 12 & $0.665769 \mathrm{E}-01$ & $0.515141 \mathrm{E}+00$ & $0.716169 \mathrm{E}-03$ & $0.146951 \mathrm{E}+00$ \\
\hline 16 & $0.581261 \mathrm{E}-01$ & $0.386489 \mathrm{E}+00$ & $0.471703 \mathrm{E}-03$ & $0.102375 \mathrm{E}+00$ \\
\hline & & & & \\
\hline
\end{tabular}

Table S5 The fitting parameters of different relaxation mechanisms for eqn (S1).

\begin{tabular}{llcc}
\hline \multirow{2}{*}{ The fitting parameters } & Meanings & Values & Values \\
& & in $\mathbf{1}$ & in 3 \\
\hline$\tau$ & the inverse of the ac frequency & $/$ & $/$ \\
$T$ & the temperature of the maximum in the ac signal & $2.0-35.0$ & $2.0-16.0$ \\
& the effective energy barrier & $\mathrm{K}$ & $\mathrm{K}$ \\
$U_{\text {eff }}$ & & $247.3 \mathrm{~K}$ & $78.0 \mathrm{~K}$ \\
& Boltzmann's constant & 1.380649 & 1.380649 \\
& & $\times 10^{-23}$ & $\times 10^{-23}$ \\
& & $\mathrm{~J} / \mathrm{K}$ & $\mathrm{J} / \mathrm{K}$
\end{tabular}




\begin{tabular}{llcc}
$\tau_{\mathrm{QTM}}$ & the fitting parameter of the QTM process & $0.0072 \mathrm{~s}$ & $0.0018 \mathrm{~s}$ \\
$n$ & the fitting parameter of the Raman process & 3.89 & 1.94 \\
& the fitting parameter of the Raman process & $0.01 \mathrm{~s}^{-1}$ & $6.41 \mathrm{~s}^{-1}$ \\
& $\mathrm{~K}^{-3.89}$ & $\mathrm{~K}^{-1.94}$ \\
$\tau_{0}$ & $1.16 \times$ & $6.74 \times$ \\
\hline
\end{tabular}

\section{Theoretical methods and computational details}

Multiconfigurational ab initio calculations, including spin-orbit coupling (SOC), were performed on the experimental structures of the complexes here to explore their SMM propertiesy. This type of calculation includes two steps: ${ }^{1}$ 1) a set of spin eigenstates, are obtained by the state-averaged (SA) CASSCF method; ${ }^{2}$ 2) the low-lying SOC states, i.e., Kramers doublets (KD) herein, are obtained by state interaction which is the diagonalization of the SOC matrix in the space spanned by the spin eigenstates from the first step. In the CASSCF step, the active space consisted of 9 electrons in 7 orbitals and all the spin eigenstates of 21 sextets were included. Due to the hardware limitation, other highly excited quartets and doublets were not considered. The step of state interaction were performed by the RASSI-SO module ${ }^{3}$ with the SOC integrals from the AMFI method. ${ }^{4}$ The ANO-RCC basis sets, ${ }^{5-7}$ including VTZP for Dy and Lu, VDZ for C and $\mathrm{H}$ as well as VDZP for other atoms, were used. All the calculations were carried out with the MOLCAS@UU, a version of MOLCAS $8.0^{10,11}$ which is freely distributed for academic users. The SINGLE ANISO module ${ }^{8,9}$ developed by Chibotaru and et al, was used to obtain the g-tensors, transition magnetic moments and other parameters characterizing the magnetic anisotropy. 

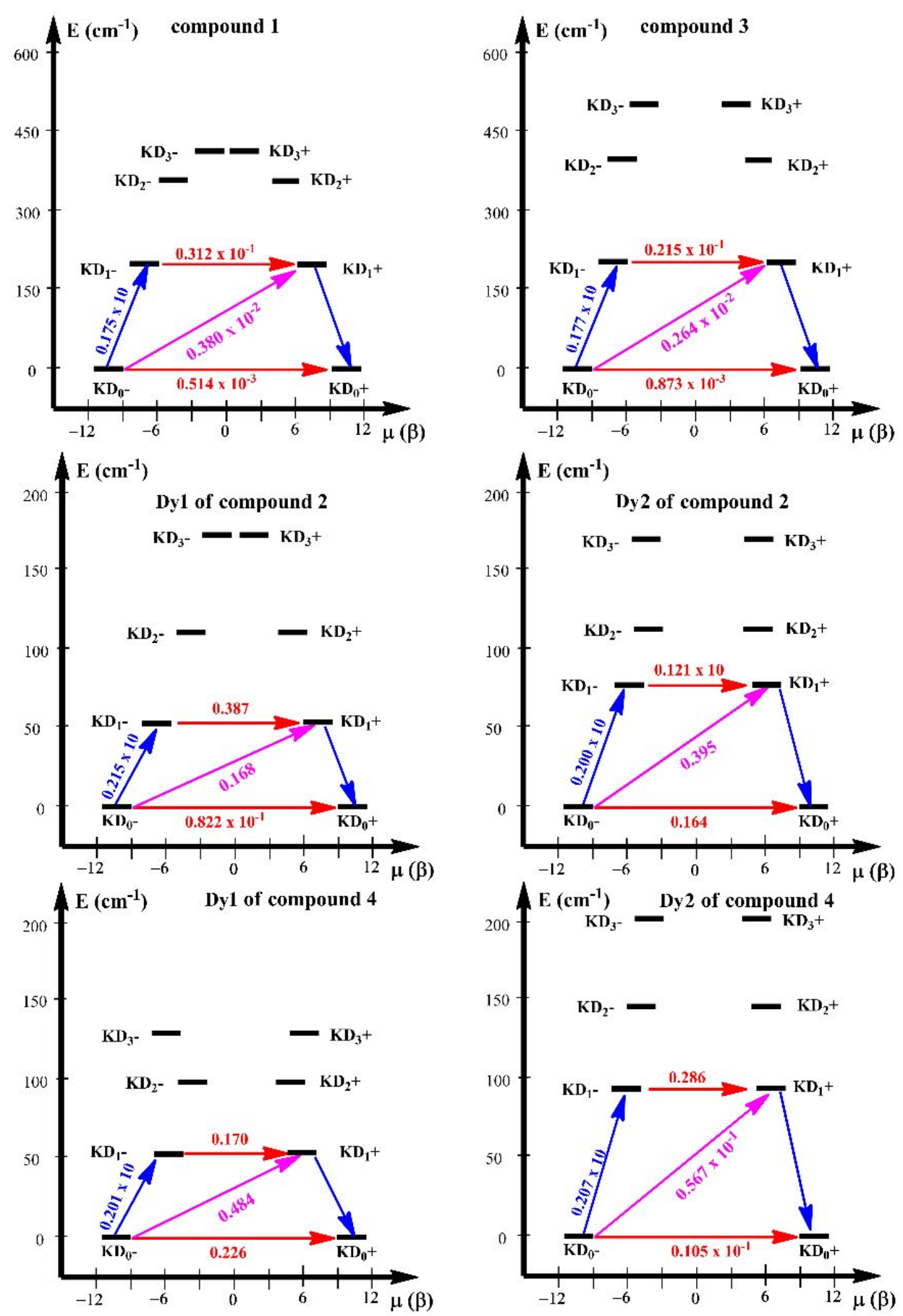

Figure S41. Qualitative description on the relaxation mechanisms from ab initio calculation (The red arrow indicates the possibility of QTM, the blue and pink arrows indicate the possibilities of other spin-lattice pathways, the numbers are the transition magnetic moment).

Table S6 Ab initio computed crystal-field parameters for the investigated compounds.

\begin{tabular}{llll}
\hline & \multicolumn{1}{c}{ 1 } & \multicolumn{1}{c}{ 2-Dy1 } & \multicolumn{1}{c}{ 2-Dy2 } \\
\hline $\mathrm{B}(2-2)$ & $0.6169 \times 10^{+00}$ & $-0.4096 \times 10^{+00}$ & $-0.4944 \times 10^{+00}$ \\
$\mathrm{~B}(2-1)$ & $-0.1098 \times 10^{+01}$ & $0.6839 \times 10^{+00}$ & $-0.3923 \times 10^{+00}$ \\
$\mathrm{~B}(20)$ & $-0.2965 \times 10^{+01}$ & $-0.1093 \times 10^{+01}$ & $-0.1031 \times 10^{+01}$
\end{tabular}




\begin{tabular}{|c|c|c|c|}
\hline $\mathrm{B}(21)$ & $0.2649 \times 10^{+01}$ & $-0.1875 \times 10^{+01}$ & $0.1272 \times 10^{-01}$ \\
\hline $\mathrm{B}(2$ 2) & $0.9388 \times 10^{+00}$ & $0.1973 \times 10^{+01}$ & $0.7497 \times 10^{+00}$ \\
\hline $\mathrm{B}(4-4)$ & $0.1750 \times 10^{-02}$ & $-0.1606 \times 10^{-01}$ & $-0.2427 \times 10^{-01}$ \\
\hline$B(4-3)$ & $-0.3943 \times 10^{-01}$ & $0.3761 \times 10^{-01}$ & $-0.2064 \times 10^{-01}$ \\
\hline $\mathrm{B}(4-2)$ & $-0.7345 \times 10^{-02}$ & $-0.1911 \times 10^{-01}$ & $-0.1483 \times 10^{-01}$ \\
\hline $\mathrm{B}(4-1)$ & $-0.7460 \times 10^{-03}$ & $0.1413 \times 10^{-01}$ & $-0.8834 \times 10^{-02}$ \\
\hline $\mathrm{B}(40)$ & $-0.7295 \times 10^{-02}$ & $-0.5889 \times 10^{-03}$ & $-0.2740 \times 10^{-02}$ \\
\hline $\mathrm{B}(4$ 1) & $-0.1456 \times 10^{-01}$ & $-0.2005 \times 10^{-01}$ & $0.2694 \times 10^{-01}$ \\
\hline $\mathrm{B}(42)$ & $-0.1045 \times 10^{-01}$ & $0.2146 \times 10^{-01}$ & $0.1787 \times 10^{-01}$ \\
\hline $\mathrm{B}(4)$ & $-0.6014 \times 10^{-01}$ & $-0.3324 \times 10^{-01}$ & $0.5171 \times 10^{-01}$ \\
\hline $\mathrm{B}(44)$ & $-0.3102 \times 10^{-02}$ & $-0.4292 \times 10^{-02}$ & $-0.2025 \times 10^{-01}$ \\
\hline$B(6-6)$ & $0.1007 \times 10^{-03}$ & $0.2675 \times 10^{-04}$ & $-0.1049 \times 10^{-03}$ \\
\hline $\mathrm{B}(6-5)$ & $0.4021 \times 10^{-03}$ & $0.2157 \times 10^{-03}$ & $-0.4002 \times 10^{-04}$ \\
\hline $\mathrm{B}(6-4)$ & $0.4682 \times 10^{-04}$ & $-0.1551 \times 10^{-03}$ & $-0.2196 \times 10^{-03}$ \\
\hline $\mathrm{B}(6-3)$ & $-0.1182 \times 10^{-03}$ & $0.3172 \times 10^{-03}$ & $-0.3248 \times 10^{-03}$ \\
\hline $\mathrm{B}(6-2)$ & $-0.4480 \times 10^{-04}$ & $-0.2913 \times 10^{-04}$ & $0.6838 \times 10^{-04}$ \\
\hline $\mathrm{B}(6-1)$ & $0.1030 \times 10^{-03}$ & $-0.1630 \times 10^{-03}$ & $0.2960 \times 10^{-04}$ \\
\hline $\mathrm{B}\left(\begin{array}{ll}6 & 0)\end{array}\right.$ & $0.2600 \times 10^{-04}$ & $-0.1522 \times 10^{-04}$ & $-0.9227 \times 10^{-05}$ \\
\hline $\mathrm{B}\left(\begin{array}{l}6 \\
1)\end{array}\right.$ & $-0.1153 \times 10^{-04}$ & $0.3507 \times 10^{-04}$ & $-0.1356 \times 10^{-03}$ \\
\hline $\mathrm{B}(62)$ & $0.2044 \times 10^{-04}$ & $0.3215 \times 10^{-04}$ & $0.1125 \times 10^{-03}$ \\
\hline $\mathrm{B}\left(\begin{array}{l}6 \\
3\end{array}\right)$ & $-0.2162 \times 10^{-03}$ & $-0.1242 \times 10^{-03}$ & $0.3148 \times 10^{-05}$ \\
\hline $\mathrm{B}(64)$ & $-0.1741 \times 10^{-03}$ & $0.1941 \times 10^{-04}$ & $-0.5653 \times 10^{-04}$ \\
\hline $\mathrm{B}(65)$ & $0.1500 \times 10^{-03}$ & $-0.4542 \times 10^{-03}$ & $0.5305 \times 10^{-03}$ \\
\hline \multirow[t]{2}{*}{$\mathrm{B}(66)$} & $0.1057 \times 10^{-03}$ & $-0.3732 \times 10^{-04}$ & $0.3381 \times 10^{-03}$ \\
\hline & 3 & 4-Dy1 & 4-Dy2 \\
\hline $\mathrm{B}(2-2)$ & $0.2894 \times 10^{+00}$ & $0.1041 \times 10^{+01}$ & $-0.5815 \times 10^{+00}$ \\
\hline $\mathrm{B}(2-1)$ & $-0.8843 \times 10^{+00}$ & $-0.8905 \times 10^{+00}$ & $-0.1027 \times 10^{+01}$ \\
\hline $\mathrm{B}(20)$ & $-0.3594 \times 10^{+01}$ & $-0.6665 \times 10^{+00}$ & $-0.1275 \times 10^{+01}$ \\
\hline $\mathrm{B}(2$ 1) & $0.2486 \times 10^{+01}$ & $-0.1480 \times 10^{+01}$ & $0.1648 \times 10^{+01}$ \\
\hline $\mathrm{B}(2$ 2) & $0.4327 \times 10^{+00}$ & $0.4556 \times 10^{+00}$ & $0.2203 \times 10^{+01}$ \\
\hline $\mathrm{B}(4-4)$ & $0.2675 \times 10^{-02}$ & $0.1402 \times 10^{-01}$ & $0.1430 \times 10^{-02}$ \\
\hline $\mathrm{B}(4-3)$ & $-0.6544 \times 10^{-02}$ & $-0.3550 \times 10^{-01}$ & $-0.6568 \times 10^{-01}$ \\
\hline $\mathrm{B}(4-2)$ & $-0.4576 \times 10^{-02}$ & $0.1633 \times 10^{-01}$ & $-0.2657 \times 10^{-01}$ \\
\hline $\mathrm{B}(4-1)$ & $0.5982 \times 10^{-03}$ & $-0.8935 \times 10^{-02}$ & $-0.1890 \times 10^{-01}$ \\
\hline $\mathrm{B}(40)$ & $-0.6841 \times 10^{-02}$ & $-0.2242 \times 10^{-02}$ & $-0.3267 \times 10^{-02}$ \\
\hline $\mathrm{B}(4$ 1) & $-0.1320 \times 10^{-01}$ & $-0.2494 \times 10^{-01}$ & $0.1041 \times 10^{-01}$ \\
\hline $\mathrm{B}(42)$ & $-0.7947 \times 10^{-02}$ & $0.1956 \times 10^{-01}$ & $0.7942 \times 10^{-02}$ \\
\hline $\mathrm{B}(4)$ 3) & $-0.8593 \times 10^{-01}$ & $-0.3435 \times 10^{-01}$ & $-0.2467 \times 10^{-01}$ \\
\hline $\mathrm{B}(44)$ & $-0.9761 \times 10^{-02}$ & $-0.2477 \times 10^{-01}$ & $-0.1654 \times 10^{-02}$ \\
\hline $\mathrm{B}(6-6)$ & $-0.2308 \times 10^{-04}$ & $0.1779 \times 10^{-03}$ & $0.1111 \times 10^{-03}$ \\
\hline $\mathrm{B}(6-5)$ & $0.1033 \times 10^{-03}$ & $-0.2055 \times 10^{-03}$ & $-0.3271 \times 10^{-03}$ \\
\hline$B(6-4)$ & $0.1082 \times 10^{-03}$ & $0.2021 \times 10^{-03}$ & $-0.1125 \times 10^{-03}$ \\
\hline $\mathrm{B}(6-3)$ & $-0.2833 \times 10^{-04}$ & $-0.3432 \times 10^{-03}$ & $-0.1517 \times 10^{-03}$ \\
\hline $\mathrm{B}(6-2)$ & $-0.5710 \times 10^{-04}$ & $-0.2123 \times 10^{-04}$ & $-0.6428 \times 10^{-04}$ \\
\hline
\end{tabular}




\begin{tabular}{|c|c|c|c|}
\hline $\mathrm{B}(6-1)$ & $0.5050 \times 10^{-04}$ & $0.6730 \times 10^{-04}$ & $0.2080 \times 10^{-03}$ \\
\hline 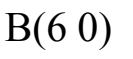 & $0.3329 \times 10^{-04}$ & $-0.9680 \times 10^{-05}$ & $-0.6434 \times 10^{-05}$ \\
\hline $\mathrm{B}(6$ 1) & $-0.2243 \times 10^{-04}$ & $0.1403 \times 10^{-03}$ & $-0.1300 \times 10^{-03}$ \\
\hline $\mathrm{B}(62)$ & $0.3040 \times 10^{-04}$ & $0.1233 \times 10^{-03}$ & $0.7311 \times 10^{-05}$ \\
\hline B(6 3) & $-0.2787 \times 10^{-03}$ & $0.1467 \times 10^{-04}$ & $-0.1657 \times 10^{-03}$ \\
\hline $\mathrm{B}(64)$ & $-0.1260 \times 10^{-03}$ & $-0.6414 \times 10^{-04}$ & $-0.9158 \times 10^{-04}$ \\
\hline B(6 5) & $0.2903 \times 10^{-03}$ & $-0.4351 \times 10^{-03}$ & $-0.4349 \times 10^{-03}$ \\
\hline $\mathrm{B}(66)$ & $0.1523 \times 10^{-03}$ & $0.2322 \times 10^{-03}$ & $-0.1092 \times 10^{-03}$ \\
\hline
\end{tabular}

\section{The PLATON squeeze process details of compound 2}

The PLATON squeeze process details of compound 2

\# SQUEEZE RESULTS $($ Version $=280120)$

\# Note: Data are Listed for all Voids in the P1 Unit Cell

\# i.e. Centre of Gravity, Solvent Accessible Volume,

\# Recovered number of Electrons in the Void and

\# Details about the Squeezed Material

loop

_platon_squeeze_void_nr

_platon_squeeze_void_average_x

_platon_squeeze_void_average_y

_platon_squeeze_void_average_z

_platon_squeeze_void_volume

_platon_squeeze_void_count_electrons

_platon_squeeze_void_content

$\begin{array}{llllll}1 & 0.006 & 0.000 & 0.500 & 970 & 285^{\prime \prime}\end{array}$

$2-0.010 \quad 0.500 \quad 0.000 \quad 970 \quad 285^{\prime \prime}$

\# Missing Reflections Below sin(th)/lambda $=0.25$

loop_

_platon_missing_refln_index_h

_platon_missing_refln_index_k

_platon_missing_refln_index_1

_platon_missing_refln_theta 


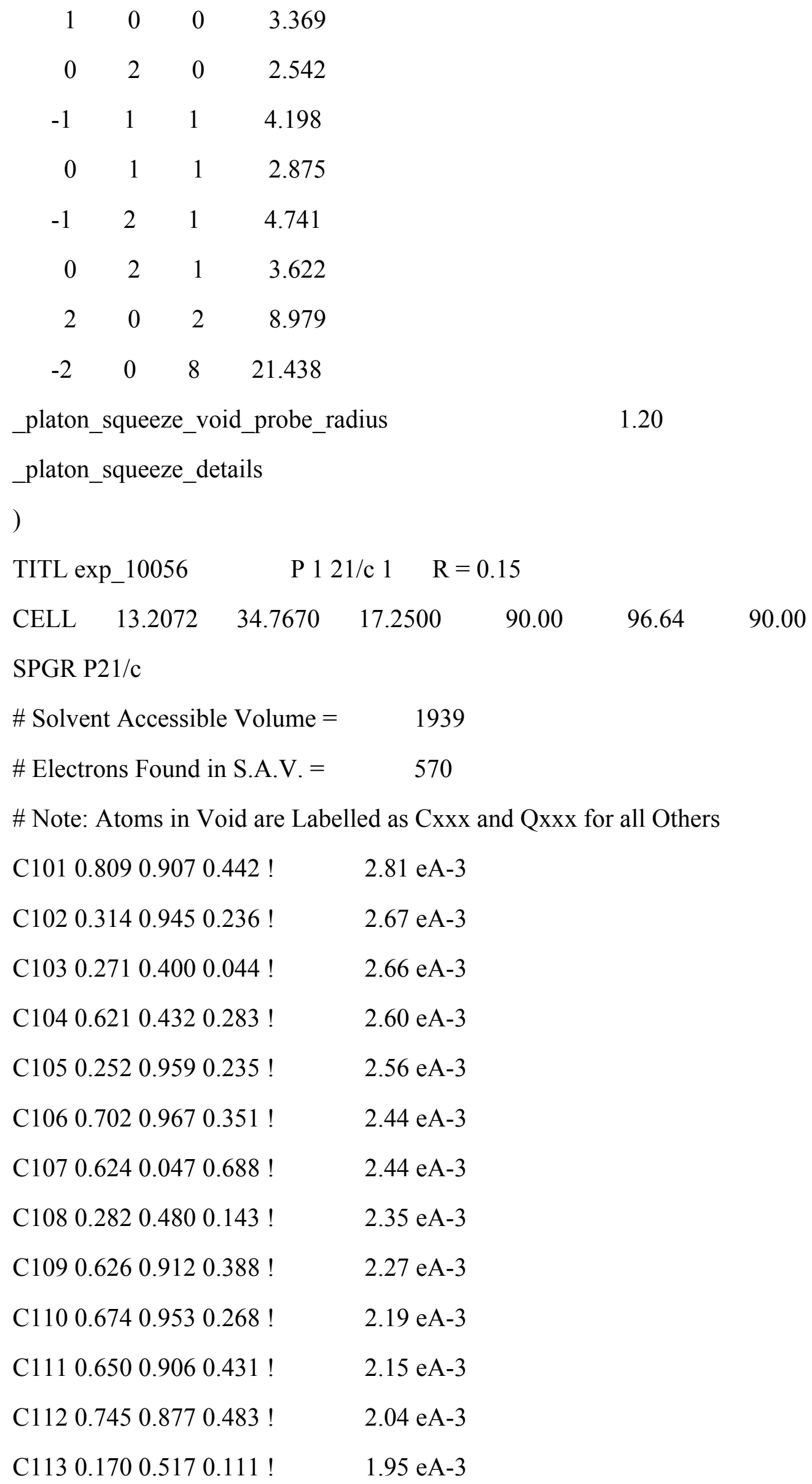




\begin{tabular}{|c|c|}
\hline C1140.606 0.0090 .387 ! & $1.77 \mathrm{eA}-3$ \\
\hline C115 0.5920 .5150 .855 ! & $1.66 \mathrm{eA}-3$ \\
\hline C116 0.221 $0.9860 .403 !$ & $1.65 \mathrm{eA}-3$ \\
\hline C117 0.544 0.006 0.406! & $1.61 \mathrm{eA}-3$ \\
\hline C118 0.262 0.516 0.155! & $1.53 \mathrm{eA}-3$ \\
\hline C119 0.6100 .9720 .482 ! & $1.49 \mathrm{eA}-3$ \\
\hline C120 0.3120 .4440 .284 ! & $1.48 \mathrm{eA}-3$ \\
\hline C121 0.1120 .5060 .105 ! & $1.47 \mathrm{eA}-3$ \\
\hline C122 0.7340 .4790 .122 ! & $1.46 \mathrm{eA}-3$ \\
\hline C1230.770 0.5680 .157 ! & $1.45 \mathrm{eA}-3$ \\
\hline C124 0.8080 .9840 .365 ! & $1.45 \mathrm{eA}-3$ \\
\hline C125 $0.6750 .9900 .391 !$ & $1.44 \mathrm{eA}-3$ \\
\hline C1260.409 0.026 0.588! & $1.43 \mathrm{eA}-3$ \\
\hline C127 0.249 0.034 0.379! & $1.41 \mathrm{eA}-3$ \\
\hline C128 $0.5650 .4740 .858 !$ & $1.37 \mathrm{eA}-3$ \\
\hline C129 0.157 0.467 0.893! & $1.36 \mathrm{eA}-3$ \\
\hline C130 $0.7900 .5560 .140 !$ & $1.35 \mathrm{eA}-3$ \\
\hline C131 0.4700 .9260 .267 ! & $1.35 \mathrm{eA}-3$ \\
\hline C132 0.8260 .5220 .133 ! & $1.34 \mathrm{eA}-3$ \\
\hline C133 0.4220 .5020 .035 ! & $1.34 \mathrm{eA}-3$ \\
\hline C134 0.222 0.019 0.406! & $1.32 \mathrm{eA}-3$ \\
\hline C135 0.2640 .4340 .282 ! & $1.31 \mathrm{eA}-3$ \\
\hline C136 0.264 0.059 0.780! & $1.31 \mathrm{eA}-3$ \\
\hline Q137 0.1730 .9700 .955 ! & $1.31 \mathrm{eA}-3$ \\
\hline C138 0.8950 .4590 .029 ! & $1.30 \mathrm{eA}-3$ \\
\hline C1390.533 0.518 0.826! & $1.30 \mathrm{eA}-3$ \\
\hline Q140 $0.8620 .3440 .746 !$ & $1.29 \mathrm{eA}-3$ \\
\hline Q141 0.6880 .4540 .894 ! & $1.27 \mathrm{eA}-3$ \\
\hline C1420.734 0.919 0.268! & $1.27 \mathrm{eA}-3$ \\
\hline Q143 0.6010 .3330 .674 ! & $1.26 \mathrm{eA}-3$ \\
\hline
\end{tabular}




\begin{tabular}{|c|c|}
\hline Q144 $0.8790 .5470 .810 !$ & $1.26 \mathrm{eA}-3$ \\
\hline Q145 $0.4580 .4160 .205 !$ & $1.23 \mathrm{eA}-3$ \\
\hline C146 0.3720 .4730 .263 ! & $1.22 \mathrm{eA}-3$ \\
\hline Q147 $0.8000 .1800 .300 !$ & $1.21 \mathrm{eA}-3$ \\
\hline C148 0.4020 .4680 .203 ! & $1.20 \mathrm{eA}-3$ \\
\hline C149 0.8930 .0320 .388 ! & $1.20 \mathrm{eA}-3$ \\
\hline C150 0.1290 .0050 .388 ! & 1.18 eA-3 \\
\hline Q151 0.7820 .0540 .325 ! & $1.17 \mathrm{eA}-3$ \\
\hline C152 0.4190 .5070 .203 ! & $1.17 \mathrm{eA}-3$ \\
\hline Q153 0.294 $0.6320 .549 !$ & $1.16 \mathrm{eA}-3$ \\
\hline C154 0.8740 .0600 .485 ! & $1.16 \mathrm{eA}-3$ \\
\hline Q155 0.912 0.202 0.066 ! & $1.16 \mathrm{eA}-3$ \\
\hline Q156 0.3440 .0240 .248 ! & $1.16 \mathrm{eA}-3$ \\
\hline C157 0.3110 .5020 .078 ! & $1.15 \mathrm{eA}-3$ \\
\hline C158 $0.6050 .4410 .117 !$ & $1.14 \mathrm{eA}-3$ \\
\hline Q159 $0.2460 .6800 .450 !$ & $1.14 \mathrm{eA}-3$ \\
\hline Q160 0.1370 .6610 .048 ! & $1.14 \mathrm{eA}-3$ \\
\hline C161 $0.4040 .4310 .140 !$ & $1.14 \mathrm{eA}-3$ \\
\hline Q162 $0.2950 .0570 .250 !$ & $1.14 \mathrm{eA}-3$ \\
\hline C163 0.1920 .4400 .253 ! & $1.14 \mathrm{eA}-3$ \\
\hline Q164 0.8940 .7140 .373 ! & $1.14 \mathrm{eA}-3$ \\
\hline C165 0.1850 .4610 .085 ! & $1.13 \mathrm{eA}-3$ \\
\hline C166 $0.4380 .5050 .889 !$ & $1.13 \mathrm{eA}-3$ \\
\hline Q167 0.7870 .3540 .153 ! & $1.13 \mathrm{eA}-3$ \\
\hline Q168 $0.4460 .6820 .519 !$ & $1.13 \mathrm{eA}-3$ \\
\hline C169 0.302 0.944 0.357! & $1.13 \mathrm{eA}-3$ \\
\hline C170 $0.4250 .4420 .207 !$ & $1.11 \mathrm{eA}-3$ \\
\hline Q171 $0.1680 .6230 .210 !$ & $1.11 \mathrm{eA}-3$ \\
\hline Q172 0.6700 .3430 .472 ! & $1.10 \mathrm{eA}-3$ \\
\hline Q173 0.1270 .6650 .314 ! & $1.10 \mathrm{eA}-3$ \\
\hline
\end{tabular}




\begin{tabular}{|c|c|}
\hline C174 0.0480 .0340 .437 ! & $1.10 \mathrm{eA}-3$ \\
\hline Q175 $0.8630 .9310 .519 !$ & $1.10 \mathrm{eA}-3$ \\
\hline Q176 0.6260 .1060 .175 ! & $1.09 \mathrm{eA}-3$ \\
\hline C177 0.7920 .5200 .770 ! & $1.09 \mathrm{eA}-3$ \\
\hline C178 0.2080 .4800 .230 ! & $1.09 \mathrm{eA}-3$ \\
\hline C179 0.966 0.515 0.145! & $1.09 \mathrm{eA}-3$ \\
\hline C180 $0.4320 .9680 .353 !$ & $1.09 \mathrm{eA}-3$ \\
\hline Q181 $0.0340 .5500 .076 !$ & $1.08 \mathrm{eA}-3$ \\
\hline Q182 $0.7660 .3890 .357 !$ & $1.08 \mathrm{eA}-3$ \\
\hline Q183 0.6400 .6030 .124 ! & $1.07 \mathrm{eA}-3$ \\
\hline C184 0.201 $0.4540 .140 !$ & $1.07 \mathrm{eA}-3$ \\
\hline C185 0.535 0.939 0.220! & $1.06 \mathrm{eA}-3$ \\
\hline Q186 0.3580 .9170 .304 ! & $1.05 \mathrm{eA}-3$ \\
\hline C187 $0.2830 .4410 .220 !$ & $1.04 \mathrm{eA}-3$ \\
\hline Q188 0.9340 .1720 .044 ! & $1.04 \mathrm{eA}-3$ \\
\hline Q189 0.7370 .0710 .221 ! & $1.04 \mathrm{eA}-3$ \\
\hline C190 0.797 0.5410.043! & $1.04 \mathrm{eA}-3$ \\
\hline Q191 $0.4870 .4020 .339 !$ & $1.03 \mathrm{eA}-3$ \\
\hline C192 $0.2800 .4420 .129 !$ & $1.03 \mathrm{eA}-3$ \\
\hline Q193 0.6620 .9770 .171 ! & $1.03 \mathrm{eA}-3$ \\
\hline C194 0.110 0.536 0.053! & $1.03 \mathrm{eA}-3$ \\
\hline C195 0.4520 .0600 .846 ! & $1.03 \mathrm{eA}-3$ \\
\hline Q196 0.255 0.594 0.286! & $1.02 \mathrm{eA}-3$ \\
\hline Q197 $0.9580 .4380 .001 !$ & $1.02 \mathrm{eA}-3$ \\
\hline Q198 0.5970 .1390 .173 ! & $1.02 \mathrm{eA}-3$ \\
\hline Q199 $0.2680 .6850 .981 !$ & $1.02 \mathrm{eA}-3$ \\
\hline \multicolumn{2}{|l|}{ ) } \\
\hline \multicolumn{2}{|l|}{; } \\
\hline _shelx_fab_checksum & 40348 \\
\hline _platon_squeeze_details & $?$ \\
\hline
\end{tabular}




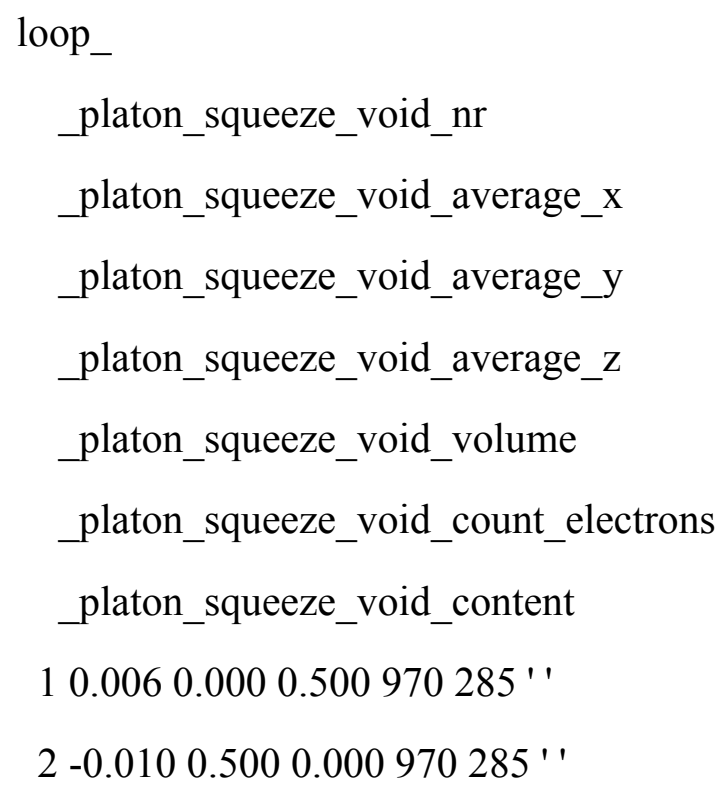

\section{References}

(1) Luzon, J.; Sessoli, R. Lanthanides in molecular magnetism: so fascinating, so challenging. Dalton Trans. 2012, 41, 13556-13567.

(2) Roos, B. O.; Taylor, P. R.; Siegbahn, P. E. M. A complete active space SCF method (CASSCF) using a density matrix formulated super-CI approach. Chem. Phys. 1980, 48, 157-173.

(3) Malmqvist, P.; Roos, B. O.; Schimmelpfennig, B. The restricted active space (RAS) state interaction approach with spin-orbit coupling. Chem. Phys. Lett. 2002, $357,230-240$.

(4) Hess, B. A.; Marian, C. M.; Wahlgren, U.; Gropen, O. A mean-field spin-orbit method applicable to correlated wavefunctions. Chem. Phys. Lett. 1996, 251, 365371.

(5) Roos, B. O.; Lindh, R.; Malmqvist, P.; Veryazov, V.; Widmark, P. Main group atoms and dimers studied with a new relativistic ANO basis set. J. Phys. Chem. A. 2004, 108, 2851-2858.

(6) Roos, B. O.; Lindh, R.; Malmqvist, P.; Veryazov, V.; Widmark, P. New relativistic ANO basis sets for transition metal atoms. J. Phys. Chem. A. 2005, 109, 6575-6579.

(7) Roos, B. O.; Lindh, R.; Malmqvist, P.; Veryazov, V.; Widmark, P.; Borin, A. C. 
New relativistic atomic natural orbital basis sets for lanthanide atoms with applications to the Ce diatom and $L u F_{3}$. J. Phys. Chem. A. 2008, 112, 11431-11435.

(8) Chibotaru, L. F.; Ungur, L. Ab initio calculation of anisotropic magnetic properties of complexes. I. Unique definition of pseudospin Hamiltonians and their derivation. J. Chem. Phys. 2012, 137, 064112.

(9) Chibotaru, L. F. Ab initio methodology for pseudospin Hamiltonians of anisotropic magnetic complexes. Adv. Chem. Phys. 2013, 153, 397-519.

(10) Aquilante, F.; De Vico, L.; Ferre, N.; Ghigo, G.; Malmqvist, P. A.; Neogrady, P.; Pedersen, T. B.; Pitonak, M.; Reiher, M.; Roos, B. O.; Serrano-Andres, L.; Urban, M.; Veryazov, V.; Lindh, R. Molcas 7: The next generation. J. Comput. Chem. 2010, 31, $224-247$.

(11) Aquilante, F.; Autschbach, J.; Carlson, R. K.; Chibotaru, L. F.; Delcey, M. G.; Vico, L. D.; Galvan, I. F.; Ferre, N.; Frutos, L. M.; Gagliardi, L.; Garavelli, M.; Giussani, A.; Hoyer, C. E.; Manni, G. L.; Lischka, H.; Ma, D.; Malmqvist, P. A.; Muller, T.; Nenov, A.; Olivucci, M.; Pedersen, T. B.; Peng, D.; Plasser, F.; Pritchard, B.; Reiher, M.; Rivalta, I.; Schapiro, I.; Segarra-Marti, J.; Stenrup, M.; Truhlar, D. G.; Ungur, L.; Valentini, A.; Vancoillie, S.; Veryazov, V.; Vysotskiy, V. P.; Weingart, O.; Zapata, F.; Lindh, R. Molcas 8: New capabilities for multiconfigurational quantum chemical calculations across the periodic table. J. Comput. Chem. 2016, 37, $506-541$. 